$\underline{\text { Preprint typeset in JHEP style. - PAPER VERSION }}$

Bicocca-FT-00/17

LPT-Orsay-01-04

Pavia-FNT/T-00/19

hep-ph/0101205

January 2001

\title{
Non-perturbative QCD analysis of near-to-planar three-jet events
}

\author{
A. Banfi, \\ Dipartimento di Fisica, Università di Milano-Bicocca and INFN, Sezione di \\ Milano, Italy
}

Yu.L. Dokshitzer,

LPT, Université de Paris XI, Centre d'Orsay, France *

\section{G. Marchesini,}

Dipartimento di Fisica, Università di Milano-Bicocca and INFN, Sezione di Milano, Italy

G. Zanderighi.

Dipartimento di Fisica, Università di Pavia and INFN, Sezione di Pavia, Italy

\begin{abstract}
We present the analysis of the $1 / Q^{-}$-suppressed non-perturbative (NP) contribution to the cumulative out-of-event-plane momentum distribution in $e^{+} e^{-}$ annihilation in the near-to-planar three-jet region. It complements our previous study of the perturbative (PT) distributions resummed to single logarithmic accuracy. Due to inter-jet soft gluon radiation, the NP contributions (as well as the PT distributions) are sensitive to the geometry (the angles between jets) and the colour structure of the underlying hard process (topology of the three-prong parton antenna). The results and techniques presented here could be extended to other multi-jet processes and, in particular, to hadron-hadron collisions.
\end{abstract}

KeYwords: QCD, Jets, LEP and SLC Physics, NLO Computations, Nonperturbative Effects.

*on leave from PNPI, Gatchina, St. Petersburg, Russia 


\section{Contents}

1. Introduction 2

2. Distributions and resummation 4

2.1 Parton kinematics and the observable 5

2.2 Resummed distribution for $K_{\text {out }} \ll Q \quad 6$

2.3 NP correction to the mean 9

$2.4 K_{\text {out }}^{T}$ case: NP radiator and recollection of PT results 10

$\begin{array}{lll}2.4 .1 & K_{\text {out }}^{T} \text {-radiator } & 10\end{array}$

2.4.2 Recollection of the PT distribution in $K_{\text {out }}^{T} \quad 13$

$2.5 K_{\text {out }}^{R}$ case: NP radiator and recollection of PT results 14

$\begin{array}{lll}2.5 .1 & K_{\text {out }}^{R} \text {-radiator } & 14\end{array}$

2.5.2 Recollection of the PT distribution in $K_{\text {out }}^{R} \quad 16$

3. NP correction to the $K_{\text {out }}^{T}$ distribution and mean $\quad 17$

3.1 Correction to the mean $K_{\text {out }}^{T} \quad 21$

3.2 The distribution $\quad 22$

4. NP correction for the $K_{\text {out }}^{R}$ distribution and mean 25

5. Summary of relevant formulae and discussion $\quad 27$

5.1 PT results 28

5.2 NP result 29

5.3 Final considerations 30

A. Soft radiation resummation and PT distributions 32

A.1 $K_{\text {out }}^{T}$ case: source and PT radiator $\quad 33$

A.2 $K_{\text {out }}^{R}$ case: source and PT radiator 36

B. NP correction to the radiators $\quad 36$

B.1 Recollection of two-jet case 36

B.2 Sudakov variables and soft distribution for the $a b$-dipole 38

B.3 Source with recoil for the $K_{\text {out }}^{T}$ distribution 39

B.4 NP radiator for the $K_{\text {out }}^{T}$ distribution 41

B.5 NP corrections to the $K_{\text {out }}^{R}$ distribution 44

B.6 Recoil integration over the NP radiator 45

C. The NP functions $\tilde{f}_{a} \quad 45$

$\begin{array}{lll}\text { C.1 } & \tilde{f}_{1} & 45\end{array}$

$\begin{array}{lll}\text { C.2 } & \tilde{f}_{2} & 47\end{array}$ 
D.1 $E_{1} \quad 50$

D.2 $E_{a}$ for $a=2,3 \quad 50$

\section{Introduction}

Multi-jet ensembles are typical for hard hadron-hadron collisions. Production of large- $p_{t}$ hadrons has mainly the topology of a four-jet event; in $W^{ \pm}, Z^{0}$ or large- $p_{t}$ photon production there are three hard jets: two formed by the initial state radiation and one from the hard parton recoiling against the electro-weak boson.

As a first step in improving the QCD description of processes involving more than two jets in the final state, we study three-jet hadronic systems in a simpler environment - without hadrons in the initial state. In this paper we continue the QCD analysis [1] of near-to-planar three-jet events in $e^{+} e^{-}$annihilation into hadrons in the regime

$$
T \sim T_{M} \gg T_{m}=\frac{K_{\text {out }}}{Q}
$$

where $T, T_{M}$, and $T_{m}$ are the thrust, the thrust major and the thrust minor, respectively.

We study the distribution in $K_{\text {out }}$ in three-jet events and aim to reach the same theoretical accuracy that is available these days for the description of the typical $e^{+} e^{-}$ hadronic systems, that is two-jet events. The collinear and infrared safe (CIS) distributions in thrust T, $C$-parameter, jet masses $M^{2}$ and broadenings $B$ in the region of two narrow jets $\left(1-T, C, M^{2} / Q^{2}, B \ll 1\right)$ have been intensively studied in recent years. Both the perturbative (PT) contribution and the leading non-perturbative (NP) corrections have been computed at high accuracy. The PT results [2] involve all-order resummation of double- (DL) and single-logarithmic (SL) contributions and matching of the approximate resummed expressions with the exact second order matrix elements. The leading NP corrections [3]-[8] have been computed at two-loop order to take into account effects of non-inclusiveness of jet observables. The $1 / Q_{-}$ suppressed NP power corrections are needed not only to make quantitative predictions of event shapes but to expand our knowledge of hadronization phenomena and of the interaction in the confinement region in general.

In [1] we have computed the SL-resummed PT expression for the integrated distribution $\Sigma\left(K_{\text {out }}\right)$ (the $K_{\text {out }}$-spectrum integrated up to a given $K_{\text {out }}$ ) in the three-jet region (1.1). We considered the cumulative out-of-plane momentum both in the total phase space $\left(K_{\text {out }}^{T}\right)$ and in the restricted right region $\left(K_{\text {out }}^{R}\right)$, that is in the narrowjet hemisphere. The results turned out to be quite interesting and informative. In 
particular at the SL-level, the PT-distribution was found to be sensitive both to the event geometry (the angles between jets) and the colour structure of the underlying hard event (specific hard parton configuration).

In this paper we study the leading NP corrections to the above PT results. The NP contributions originate from the part of the phase space that corresponds to emission of secondary gluons from "large" distances, where the QCD coupling runs into trouble and the PT treatment becomes questionable. Our present understanding of QCD does not provide a "fundamental" solution to the problem. For a CIS observable, however, this part of the phase space does not lead to divergences (neither collinear nor soft), but gives rise to a genuine NP contribution. The latter cannot be calculated from "first principles". The only firm information we have is that longdistance contributions bring in corrections to the PT results that are suppressed as an inverse power of the hard scale $Q$. The exponent $p$ of the suppression factor $Q^{-p}$ can be extracted from the analysis of the divergence of the formal PT series (renormalon analysis, for a recent review see [4]).

To make quantitative predictions and to make it possible to compare NP contributions to different observables, it has been suggested [3] to assume that the running coupling can still be defined at large distances. This simple assumption allows one to give meaning to PT contributions at any order and to relate the magnitudes of the power corrections with momentum averages of the QCD coupling in the infrared region.

We follow the dispersive method [8] which extends the notion of the QCD coupling to small momentum scales and helps to extract and quantify the large-distance contribution to a given CIS observable. We show that the leading NP correction to $K_{\text {out }}$ distributions and means belongs to the same class as the two-jet shape observables listed above [5].

The main results, both in PT and NP sectors, were announced and their physical aspects anticipated in a short letter [6]. In this paper we present the derivation of the NP contributions to $K_{\text {out }}^{T / R}$-distributions and means.

The results for the $K_{\text {out }}$-distributions that are common to the $1-T, M^{2} / Q^{2}, C$ and $B$ distributions are:

- the leading NP corrections are of order $1 / Q$. Since the actual expansion parameter is $1 / K_{\text {out }}$ rather than $1 / Q$, they are leading as long as $K_{\text {out }}$ is larger than $\Lambda_{\mathrm{QCD}}$. For $K_{\text {out }}$ of the order of $\Lambda_{\mathrm{QCD}}$, higher powers become equally important $[9]$

- the leading NP corrections in the region $\Lambda_{\mathrm{QCD}} \ll K_{\text {out }} \ll Q$ can be embodied in a shift $\delta K_{\text {out }}$ of the argument of the PT distributions;

- the shift is a logarithmic function of $K_{\text {out }}$ and is determined analytically by perturbative calculations, except for the overall normalization parameter $\lambda^{\mathrm{NP}}$; 
- the phenomenological NP parameter $\lambda^{\mathrm{NP}}$ is given by the momentum integral of the QCD coupling in the infrared region. It is the same parameter that determines NP corrections to the two-jet shape observables $\left(T, M^{2}, C, B\right)$.

Specific features of the $K_{\mathrm{out}}^{T / R}$-distributions are the following:

- for the right $K_{\text {out }}^{R}$-distribution, the shift behaves logarithmically as $\ln \frac{Q^{\mathrm{NP}}}{K_{\text {out }}}$, which behaviour is similar to that of the single-jet broadening [7];

- for the total $K_{\text {out }}^{T}$-distribution, the logarithmic contribution $\ln \frac{Q^{\mathrm{NP}}}{K_{\text {out }}}$ is supplemented with a singular constant term proportional to $1 / \sqrt{\alpha_{\mathrm{s}}}$ which contribution has a weird colour structure and dominates at moderately small $K_{\text {out }}$. This behaviour is due to an interplay between PT and NP effects and is reminiscent of the total broadening case [7];

- the relevant hard scales $Q^{\mathrm{NP}}$ are affected both by the small- and large-angle radiation of secondary partons. For the $K_{\text {out }}^{T}$ distribution, these scales have a simple geometrical interpretation. As a result, the NP corrections, as well as the PT distributions, depend on geometry and colour structure of the hard event.

A certain similarity between the $K_{\text {out }}$ and the broadening distribution is due to the fact that both these observables accumulate contributions from arbitrarily large soft parton rapidities (while $T, M^{2}, C$ are dominated by finite rapidities - largeangle radiation only). As found in [7], it is this feature that is responsible for the logarithmic behaviour and the singular $1 / \sqrt{\alpha_{\mathrm{s}}}$ contributions to the shift.

The need to present reproduceable derivations can sometimes be detrimental to readability of a paper. This is especially true for the present topic where the tricky event-plane kinematics and the NLO accuracy lead to technical complications. We have nevertheless tried our best to keep the paper readable.

In section 2 we set up the kinematics, recall the PT results that will be needed for the NP analysis, and describe the NP corrections to the Sudakov exponents (radiators). Sections 3 and 4 contain the new results of this study, namely the NP corrections to the distributions and means of $K_{\text {out }}^{T}$ and $K_{\text {out }}^{R}$ observables. In section 5 we summarize the results and discuss their physical properties. The detailed calculations are presented in a series of Appendices.

\section{Distributions and resummation}

In the near-to-planar three-jet region (1.1) the event, at parton level, can be treated as a hard quark-antiquark-gluon system accompanied by an ensemble of secondary partons $k_{i}$. We denote by $p_{a}(a=1,2,3)$ the three energy-ordered hard parton momenta: $p_{1}>p_{2}>p_{3}$. 
Defining the event plane as the $\{y z\}$-plane (we set the thrust axis and the thrustmajor axis equal to the $z$ - and $y$-axis, respectively), the total out-of-plane momentum variable is defined by

$$
K_{\text {out }}^{T}=\sum_{a=1}^{3}\left|p_{a x}\right|+\sum_{i=1}^{n}\left|k_{i x}\right| .
$$

We consider also the observable restricted to the right region $(R)$, that is the hemisphere with the lowest broadening:

$$
K_{\text {out }}^{R}=\left|p_{1 x}\right|+\sum_{i \in R}\left|k_{i x}\right| .
$$

The hardest parton $p_{1}$ lies in the right hemisphere, near the thrust axis.

In this section we first set up the kinematics of partons in the region (1.1) and define the event plane. We then recall the PT results that are needed for the $\mathrm{NP}$ analysis, in particular the factorization of soft emission and the result of SL resummation (details are reported in Appendix A). We finally report and discuss the NP corrections to the Sudakov exponents (radiators) that are evaluated in Appendix B.

\subsection{Parton kinematics and the observable}

At Born level one considers only the hard $q \bar{q}, g$ system and neglects the soft radiation so that $K_{\text {out }}=0$. We denote the three hard massless Born momenta by $P_{a}$, take $P_{1}$ along the thrust axis and $P_{2}$ more energetic than $P_{3}$, see Fig. 1. Given $T$ and $T_{M}$ we have $^{2}$

$$
\begin{aligned}
& P_{1}=E(T, 0,0, T), \quad P_{2}=E\left(x_{2}, 0, T_{M},-t_{2}\right), \quad P_{3}=E\left(x_{3}, 0,-T_{M},-t_{3}\right), \\
& x_{2}=\frac{2-T}{2}+\frac{T}{2} \rho, \quad t_{2}=\frac{T}{2}+\frac{2-T}{2} \rho, \quad \rho \equiv \sqrt{1-\frac{T_{M}^{2}}{1-T}}, \quad E=\frac{Q}{2} .
\end{aligned}
$$

The fractions $x_{3}$ and $t_{3}$ are obtained from $2=T+x_{2}+x_{3}$ and $T=t_{2}+t_{3}$.

There are essentially three underlying configurations for the hard partons according to which is the parton momentum corresponding to the gluon. We denote by $\delta$ the configuration in which the momentum of the hard gluon is $P_{\delta}$. In Fig. 1 we represent the three Born configurations.

Beyond the Born level, the three hard parton momenta $p_{a}$ no longer lie in the plane but are displaced from $P_{a}$ by "soft recoils" $q_{a}$ :

$$
p_{a}=P_{a}+q_{a} .
$$

\footnotetext{
${ }^{2}$ To define the massless momenta $P_{a}$ one requires that $T$ and $T_{M}$ are restricted to the region

$$
\frac{2(1-T) \sqrt{2 T-1}}{T}<T_{M}<\sqrt{1-T} .
$$
}

In the following we consider $T, T_{M}$ restricted to this region. 


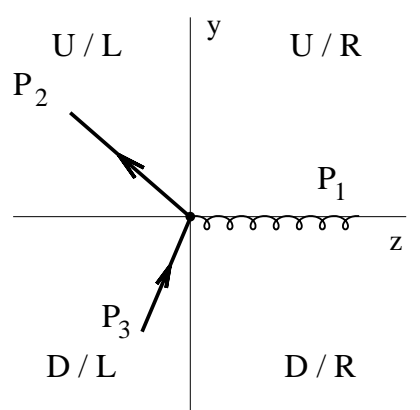

$\delta=1$

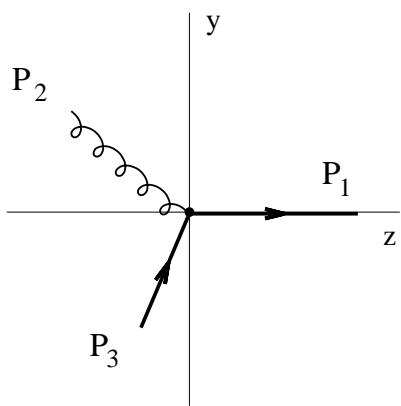

$\delta=2$

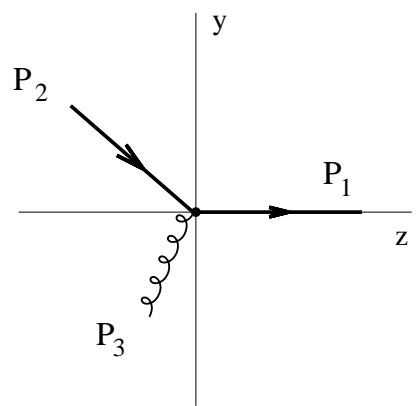

$\delta=3$

Figure 1: The three Born configurations $\delta$ for $T=0.75$ and $T_{M}=0.48$ ordered according to increasing probability. The thrust $T$ and thrust major $T_{M}$ are along the $z$ - and $y$ axis respectively. The up, down, left and right hemispheres ( $U, D, L, R$ respectively) are indicated.

As we have shown in [1], finite rescaling of the in-plane momenta $p_{a z}, p_{2 y}, p_{3 y}$, due to hard collinear splittings, gets absorbed into the first hard correction to the emission probability of soft gluons, which is then resummed and embodied into the radiator. Bearing this in mind, all recoils $q_{a}$ and the secondary parton momenta $k_{i}$ can be treated as small (soft).

Due to the fact that $p_{a z} \sim P_{a z}=\mathcal{O}(Q), p_{2 y} \sim P_{2 y}=\mathcal{O}(Q)$ and $p_{3 y} \sim P_{3 y}=\mathcal{O}(Q)$, the five soft in-plane recoil components $q_{a z}, q_{2 y}, q_{3 y}$ can be neglected both in the definition of the event plane and in the soft matrix elements. Then, the definition of the event plane involves the remaining four recoil components and results in the following kinematical constraints:

$$
\begin{aligned}
& \vec{q}_{1 t}+\sum_{R} \vec{k}_{i t}=0, \quad \vec{q}_{1 t} \equiv\left(q_{1 x}, q_{1 y}\right), \quad \vec{k}_{i t} \equiv\left(k_{i x}, k_{i y}\right) \\
& q_{2 x}+q_{1 x}^{+}+\sum_{U} k_{i x}=0, \quad q_{3 x}+q_{1 x}^{-}+\sum_{D} k_{i x}=0, \quad q_{1 x}^{ \pm} \equiv q_{1 x} \vartheta\left( \pm q_{1 y}\right)
\end{aligned}
$$

where we denote by $R, L, U$ and $D$ the right-, left-, up- and down- phase space regions, see Fig. 1. The total and the right-hemisphere $K_{\text {out }}$ become

$$
K_{\text {out }}^{T}=\left|q_{1 x}\right|+\left|q_{2 x}\right|+\left|q_{3 x}\right|+\sum_{i}\left|k_{i x}\right|, \quad K_{\text {out }}^{R}=\left|q_{1 x}\right|+\sum_{i \in R}\left|k_{i x}\right| .
$$

\subsection{Resummed distribution for $K_{\text {out }} \ll Q$}

We study the integrated $K_{\text {out-distribution defined as }}$

$$
\frac{d \sigma^{T}\left(K_{\text {out }}\right)}{d T d T_{M}}=Q^{5} \sum_{m} \int d \sigma_{m} \delta^{3}\left(\sum_{h \in R} \vec{p}_{h}-\vec{P}_{1}\right) \delta^{2}\left(\sum_{h \in U} \vec{p}_{h t}-\vec{P}_{2 t}\right) \vartheta\left(K_{\text {out }}-\sum_{h=1}^{m}\left|p_{h x}\right|\right),
$$


where $m$ denotes the number of final particles with momenta $p_{h}$. The two deltafunctions fix the event plane and the theta-function defines the observable. For the total- $K_{\text {out }}$ case $\left(K_{\text {out }}^{T}\right)$ the sum runs over all particles in the event. Analogously we define the right distribution $K_{\text {out }}^{R}$ by restricting the sum over particles in the theta-function of (2.7) to those belonging to the right hemisphere.

In the near-to-planar "three-jet region" (1.1), the distributions in $K_{\text {out }}^{T}$ or in $K_{\text {out }}^{R}$ are factorized in the form

$$
\frac{d \sigma^{T / R}\left(K_{\mathrm{out}}\right)}{d T d T_{M}}=c\left(\alpha_{\mathrm{s}}\right) \cdot \sum_{\delta=1}^{3} \frac{d \sigma_{\delta}^{(0)}}{d T d T_{M}} \cdot \Sigma_{\delta}^{T / R}\left(K_{\text {out }}\right) .
$$

The factor $d \sigma_{\delta}^{(0)} / d T d T_{M}$ is the three-jet differential Born cross section in the configuration $\delta$. The factor $\Sigma_{\delta}^{T / R}$ accounts for the soft radiation emitted by the hard $q \bar{q}, g$ system. The first factor

$$
c\left(\alpha_{\mathrm{s}}\right)=1+\mathcal{O}\left(\alpha_{\mathrm{s}}\right)
$$

is the non-logarithmic coefficient function which takes into account corrections due to hard large-angle radiation not included in $\Sigma_{\delta}^{T / R}\left(K_{\text {out }}\right)$. The distribution $\Sigma_{\delta}^{T / R}$ is given by

$$
\Sigma_{\delta}^{T / R}\left(K_{\text {out }}\right)=\sum_{n} \frac{1}{n !} \int d H_{n}^{T / R}\left(K_{\text {out }}\right) \prod_{i}^{n} \frac{d^{3} k_{i}}{\pi \omega_{i}} M_{n, \delta}^{2}
$$

Here $M_{n, \delta}^{2}$ is the distribution for the emission of $n$ soft partons from the primary $q \bar{q}, g$ system in the configuration $\delta$ and $d H_{n}^{T / R}\left(K_{\text {out }}\right)$ is (a part of) the phase space, involving the three-jet kinematics (2.5) and the observable (2.6).

The procedure used to resum the PT contributions of the $K_{\text {out }}$-distribution is described in detail in [1] and recalled in Appendix A. The essential point which allows resummation of $\Sigma_{\delta}^{T / R}\left(K_{\text {out }}\right)$ is the factorization of the soft distribution $M_{n, \delta}^{2}$ and of the phase space $d H_{n}^{T / R}\left(K_{\text {out }}\right)$. To achieve SL accuracy one needs to consider the soft emission factorization of $M_{n, \delta}^{2}$ at the two-loop level.

Resummation employs the Mellin-Fourier representation of $\Sigma_{\delta}^{T / R}\left(K_{\text {out }}\right)$ and gives rise to the exponential of the radiator. To describe this structure (see [1], Appendices $\mathrm{A}$ and $\mathrm{B}$ ), we start from the phase space factor $d H_{n}^{T}$ which is given by

$$
\begin{aligned}
& d H_{n}^{T}\left(K_{\text {out }}\right) \equiv d^{2} \vec{q}_{1 t} d q_{2 x} d q_{3 x} \vartheta\left(K_{\text {out }}-\sum_{a=1}^{3}\left|q_{\text {ax }}\right|-\sum_{i=1}^{n}\left|k_{i x}\right|\right) \\
& \times \delta^{2}\left(\vec{q}_{1 t}+\sum_{R} \vec{k}_{i t}\right) \delta\left(q_{2 x}+q_{1 x}^{+}+\sum_{U} k_{i x}\right) \delta\left(q_{3 x}+q_{1 x}^{-}+\sum_{D} k_{i x}\right),
\end{aligned}
$$


where the delta functions define the event plane (2.5). Correspondingly, the phase space factor $d H_{n}^{R}$ involves only partons emitted in the right region:

$$
d H_{n}^{R}\left(K_{\text {out }}\right) \equiv d q_{1 x} \vartheta\left(K_{\text {out }}-\left|q_{1 x}\right|-\sum_{R}\left|k_{i x}\right|\right) \delta\left(q_{1 x}+\sum_{R} k_{i x}\right) .
$$

In the latter case, since the distribution is insensitive to the soft recoil components $q_{2 x}, q_{3 x}$ and $q_{1 y}$, they can be neglected in the matrix element and integrated over.

To factorize the multi-parton emissions in the phase space factors $d H_{n}^{T / R}$ we use the Mellin and Fourier representations for the theta- and delta-functions in (2.10) and (2.11). This results in

$$
d H_{n}^{T / R}=\int \frac{d \nu e^{\nu K_{\mathrm{out}}}}{2 \pi i \nu} d h_{n}^{T / R}, \quad d h_{n}^{T / R}=\int d \mu^{T / R} \prod_{i=1}^{n} u^{T / R}\left(k_{i}\right),
$$

where the integration elements $d \mu^{T / R}$ and the parton sources $u^{T / R}\left(k_{i}\right)$ are described in Appendix A and B. Here we report some essential points concerning the integration and the sources.

Integration. For the $K_{\text {out }}^{T}$ case we need to introduce four Fourier variables $\gamma, \beta_{a}$ conjugated with $q_{1 y}$ and $q_{a x}$, respectively. For the $K_{\text {out }}^{R}$ case only one Fourier variable $\beta$ conjugated with $q_{1 x}$ is needed. The two integrations are

$$
\begin{aligned}
\int d \mu^{T} & \equiv \frac{\nu^{4}}{8} \int_{-\infty}^{\infty} \frac{d \gamma d q_{1 y}}{2 \pi} \prod_{a=1}^{3} \int_{-\infty}^{\infty} \frac{d \beta_{a} d q_{a x}}{\pi} V^{T}(\gamma, \beta, q) \\
\int d \mu^{R} & \equiv \frac{\nu}{2} \int_{-\infty}^{\infty} \frac{d \beta d q_{1 x}}{\pi} V^{R}\left(\beta, q_{1 x}\right)
\end{aligned}
$$

Here we have rescaled the Fourier variables by $\nu$, so that they are dimensionless. The functions $V^{T / R}$ are given in (A.7) and (A.20). They are the Mellin-Fourier factors involving the recoil momenta.

Sources. The soft-parton sources $u^{T / R}\left(k_{i}\right)$ contain Mellin - Fourier factors involving the secondary parton momenta. The sources enter as the factors $\left[u\left(k_{i}\right)-1\right]$ integrated with the radiation probability $d w\left(k_{i}\right)$, where the subtraction unity corresponds to the virtual contribution. The source function tends to unity, $u\left(k_{i}\right) \rightarrow 1$, when $k_{i}$ becomes collinear to one of the radiating hard partons $p_{a}$. This ensures cancellation of the singularities in the soft matrix element $M_{n, \delta}^{2}$.

As has been already mentioned, for the sake of the PT-resummation the parton recoil effects can be neglected in the soft matrix element [7], so that we can take $u\left(k_{i}\right) \rightarrow 1$ for $k_{i}$ collinear to the skeleton-momenta $P_{a}$ (see (A.6) and (A.21)). In the calculation of the NP corrections, however, it is essential to keep the recoil in the soft matrix element, so as described in detail in Appendix B. This makes it necessary 
to accurately define the sources with account of the recoil as well, as to preserve the real-virtual cancellations in the collinear limit, see (B.19) and (B.34).

The $K_{\text {out }}$-distributions share this feature with the broadenings [7]. In both cases the observables are uniform in secondary parton rapidities. In our case, accumulation of the NP contribution to $K_{\text {out }}$ over large rapidities is bounded by the mismatch between the actual parton momentum $p_{a}$ and the corresponding "Born" momentum $P_{a}$ (which lies in the event plane). Thus, the rapidity integrals turn out to be essentially the logarithm of the angle between the hard parton \#a and the event plane.

From (2.12) we obtain the Mellin representation of $\Sigma_{\delta}^{T / R}\left(K_{\text {out }}\right)$ :

$$
\Sigma_{\delta}^{T / R}\left(K_{\mathrm{out}}\right)=\int \frac{d \nu e^{\nu K_{\mathrm{out}}}}{2 \pi i \nu} \sigma_{\delta}^{T / R}(\nu),
$$

where the contour runs parallel to the imaginary axis with $\operatorname{Re} \nu>0$. The Mellin moment $\sigma_{\delta}^{T / R}(\nu)$ is given by the sum, see (2.9),

$$
\begin{aligned}
& \sigma_{\delta}^{T / R}(\nu)=\sum_{n} \frac{1}{n !} \int d h_{n}^{T / R}\left(K_{\text {out }}\right) \prod_{i}^{n} \frac{d^{3} k_{i}}{\pi \omega_{i}} M_{n, \delta}^{2} \\
& =\int d \mu^{T / R}\left\{\sum_{n} \frac{1}{n !} \int \prod_{i}^{n} \frac{d^{3} k_{i}}{\pi \omega_{i}} u^{T / R}\left(k_{i}\right) \cdot M_{n, \delta}^{2}\right\}=\int d \mu^{T / R} e^{-\mathcal{R}^{T / R}},
\end{aligned}
$$

where the radiator $\mathcal{R}^{T / R}$ at SL level is obtained by using soft emission factorization of $M_{n, \delta}^{2}$ at two loops. The radiator depends on the hard momenta $P_{a}$, the Mellin and Fourier variables and, in general, on the recoil momenta.

The Mellin moment $\sigma_{\delta}^{T / R}(\nu)$ is a regular function of $\nu$ in the entire complex plane. It decreases for $\operatorname{Re} \nu \rightarrow \infty$ so that

$$
\Sigma_{\delta}^{T / R}\left(K_{\text {out }}<0\right)=0 .
$$

The fact that $\sigma_{\delta}^{T / R}(\nu) \propto e^{\nu K_{\text {out }}^{m}}$ for $\operatorname{Re} \nu \rightarrow-\infty$, where $K_{\text {out }}^{m}$ is the maximum kinematical value of $K_{\text {out }}^{T / R}$, ensures that

$$
\Sigma_{\delta}^{T / R}\left(K_{\text {out }}>K_{\text {out }}^{m}\right)=\sigma_{\delta}^{T / R}(0)=1 .
$$

The near-to-planar kinematics $K_{\text {out }} \ll Q$ corresponds to the region of the Mellin variable $Q \nu \gg 1$.

\subsection{NP correction to the mean}

From the Mellin representation (2.14), we formally get

$$
\left\langle K_{\mathrm{out}}^{T / R}\right\rangle_{\delta} \equiv \int_{0}^{\infty} K d K \frac{d \Sigma_{\delta}^{T / R}(K)}{d K}=-\left.\partial_{\nu} \sigma_{\delta}^{T / R}(\nu)\right|_{\nu=0},
$$


where, due to (2.17), the integration over $K$ has been extended to infinity. This quantity, at the $\mathrm{PT}$ level, is determined by the region $K_{\text {out }} \sim Q$ where the soft approximation is invalid. However, the NP correction originates, in any case, from the region of small (transverse) momenta of the order of $\Lambda_{\mathrm{QCD}}$. Therefore the NP correction to the mean $K_{\text {out }}^{T / R}$ can be computed within the present formalism based on the soft factorization. The NP correction to the mean is then obtained, according to (2.18), from the NP contribution to $\sigma_{\delta}^{T / R}(\nu)$ at $\nu=0$. Even though $Q \nu=\mathcal{O}(1)$, the NP answer is still dominated by small secondary gluon momenta, that is, by large values of the Fourier variables $\beta_{a}$ and $\gamma: Q \nu \beta_{a} \sim Q \nu \gamma \gg 1$.

\section{$2.4 K_{\text {out }}^{T}$ case: NP radiator and recollection of PT results}

We discuss the structure of the PT and NP components of the radiator for the $K_{\text {out }}^{T}$ distribution. The detailed calculation of the NP corrections are given in Appendix B. We also recall the essential points of the PT results both for the radiator and the distribution (see [1] and Appendix A), which are needed to derive the NP corrections in the next section. In this subsection we suppress the index $T$ and the index $\delta$ marking the jet configuration.

\subsection{1 $K_{\text {out-radiator }}^{T}$}

The radiator for the $K_{\text {out }}^{T}$ distribution has PT and NP components:

$$
\mathcal{R}(\nu, \beta, \gamma, q)=\mathcal{R}^{\mathrm{PT}}(\nu, \beta, \gamma)+\delta \mathcal{R}(q) .
$$

The PT contribution depends on the Fourier variables but is independent of the recoil momenta. (As mentioned, recoil effects at PT level produce corrections that are beyond the SL accuracy, see [7]). On the contrary, the NP correction depends on the out-of-plane $x$-components of the recoil momenta but does not depend on $\gamma, \beta_{a}$.

We first address the NP contribution to the radiator calculated in detail in Appendix B. We have

$$
\delta \mathcal{R}(q)=\nu \lambda^{\mathrm{NP}} \sum_{a} C_{a} \ln \frac{Q_{a}^{\mathrm{NP}}}{\left|q_{a x}\right|} .
$$

We comment on this expression.

- $C_{a}$ is the colour charge of parton \#a in the configuration $\delta$ (here implicit). For instance, for the most probable configuration $\delta=3$ in which the gluon is the least energetic of the three hard partons, we have $C_{1}=C_{2}=C_{F}$ and $C_{3}=N_{c}$.

- Expression (2.20) is obtained by linearizing the sources $u^{T}\left(k_{i}\right)$. The NP parameter $\lambda^{\mathrm{NP}}$ defined in (B.30), has dimension of mass and is responsible for $1 / Q$ power corrections to jet observables. 
- Linearization makes $\delta \mathcal{R}$ proportional to the Mellin variable $\nu$ which, in turn, implies that the NP correction emerges essentially as a shift in $K_{\text {out }}$.

- The logarithmic behaviour in $\left|q_{a x}\right|$ comes from the soft gluon rapidity integration which is bounded by the rapidity of the parton $p_{a}$, with rapidities defined with respect to the corresponding Born momenta $P_{a}$. (Radiation at smaller angles corresponds to collinear splitting and does not contribute to the observable due to real-virtual cancellation.)

- The hard NP scale $Q_{a}^{\mathrm{NP}} \sim Q$ associated with hard parton \#a contains two factors:

$$
Q_{a}^{\mathrm{NP}}=Q_{a} \cdot \zeta^{\mathrm{NP}}, \quad \zeta^{\mathrm{NP}}=2 e^{-2}
$$

The rescaling factor $\zeta^{\mathrm{NP}}$ is defined in (B.27) and originates as a constant leftover after the real-virtual cancellation of collinear singularities in the NP radiation.

The first factor $Q_{a}$ is determined by large-angle soft emissions and is sensitive to the geometry of the event as it depends on the angles between jets. In the configuration $\delta$ one has

$$
Q_{b}^{2}=Q_{c}^{2}=2\left(P_{b} P_{c}\right), \quad Q_{\delta}^{2}=\frac{2\left(P_{b} P_{\delta}\right)\left(P_{\delta} P_{c}\right)}{\left(P_{b} P_{c}\right)},
$$

where $b, c \neq \delta$ denote the quark-antiquark indices. The scales for the fermions are equal to the invariant mass of the quark-antiquark dipole. For the gluon, $Q_{\delta}$ is the invariant gluon transverse momentum with respect to the $q \bar{q}$ pair. (Notice that when the gluon becomes collinear to the quark or the antiquark, the scale $Q_{\delta}$ decreases and the non-Abelian contribution reduces.)

- The NP radiator does not depend on the Fourier variables $\beta_{a}, \gamma$. As in the case of broadening, see [7], this is due to the fact that the contributions linear in $k_{x}$ or $k_{y}$ vanish, and the $\beta_{a}, \gamma$-dependence of the radiator emerges only at the $1 / Q^{2}$ level.

- Since the NP radiator depends on $q_{a x}$, the way it affects the $K_{\text {out-distribution }}$ depends on the PT distributions in parton recoils.

The NP corrections to the integrated distribution $\Sigma\left(K_{\text {out }}\right)$ that correspond to the NP radiator $\delta \mathcal{R}$ are analyzed in the next section.

Now we recall the structure of the PT radiator in (2.19) (for details see Appendix A):

$$
\begin{aligned}
& \mathcal{R}^{\mathrm{PT}}(\nu, \beta, \gamma)=\mathbf{R}_{1}\left(\bar{\nu}, \beta_{12}, \beta_{13}, \gamma\right)+R_{2}\left(\rho_{2}\right)+R_{3}\left(\rho_{3}\right) \\
& \mathbf{R}_{1}\left(\bar{\nu}, \beta_{12}, \beta_{13}, \gamma\right) \equiv \int_{0}^{\infty} \frac{d y}{\pi\left(1+y^{2}\right)}\left\{R_{1}\left(\rho_{12}\right)+R_{1}\left(\rho_{13}\right)\right\}
\end{aligned}
$$


with $\left(\beta_{1 a}=\beta_{1}+\beta_{a}\right)$

$$
\begin{aligned}
\rho_{12} & \equiv \bar{\nu} \sqrt{(1-i \gamma y)^{2}+\beta_{12}^{2}}, \quad \rho_{13} \equiv \bar{\nu} \sqrt{(1+i \gamma y)^{2}+\beta_{13}^{2}}, \\
\rho_{a} & \equiv \bar{\nu} \sqrt{1+\beta_{a}^{2}}, \quad a=2,3, \quad \bar{\nu}=e^{\gamma_{E}} \nu .
\end{aligned}
$$

The three terms of $\mathcal{R}^{\mathrm{PT}}$ in (2.23) describe the radiation from partons \#1, \#2 and \#3, respectively. The two pieces of $\mathbf{R}_{1}$ correspond to secondary gluon emission in the $\mathrm{Up}\left(k_{y}>0\right)$ and Down hemispheres $\left(k_{y}<0\right)$. The function $R_{a}(\rho)$ is the two-loop single-jet radiator given by

$$
R_{a}(\rho) \equiv C_{a} r\left(\rho, Q_{a}^{\mathrm{PT}}\right), \quad r\left(\rho, Q_{a}^{\mathrm{PT}}\right)=\frac{2}{\pi} \int_{1 / \rho}^{Q_{a}^{\mathrm{PT}}} \frac{d k_{x}}{k_{x}} \alpha_{\mathrm{s}}\left(2 k_{x}\right) \ln \frac{Q_{a}^{\mathrm{PT}}}{k_{x}},
$$

with the running coupling in the physical ("bremsstrahlung"; CMW) scheme [10]. (The factor 2 in the argument of the running coupling originates after the integration over the in-plane component of the transverse momentum.) The perturbative hard scales are again composed of two factors:

$$
Q_{a}^{\mathrm{PT}}=Q_{a} \cdot \zeta_{a}^{\mathrm{PT}}, \quad \zeta_{a}^{\mathrm{PT}}=\frac{1}{2} e^{-3 / 4} \text { for } a \neq \delta(q \text { or } \bar{q}), \quad \zeta_{a}^{\mathrm{PT}}=\frac{1}{2} e^{-\beta_{0} / 4 N_{c}} \text { for } a=\delta(g) .
$$

The first are the same geometry-dependent factors (2.22) as in the NP scales, that account for soft large-angle emission. The second factors are hard SL corrections due to collinear quark $\left(-3 C_{F}\right)$ and gluon $\left(-\beta_{0}\right)$ splittings. (The factor $1 / 2$ in $\zeta_{a}^{\mathrm{PT}}$, again, comes from the integration over the in-plane components.)

The function $r$ contains both DL $\left(\alpha_{\mathrm{s}}^{n} L^{n+1}\right)$ and SL $\left(\alpha_{\mathrm{s}}^{n} L^{n}\right)$ contributions, with $L=\ln \rho Q$ and $n \geq 1$. The precise value of the hard scale and, in particular, the geometry of the event, is relevant only at SL level.

The same is true for the dependence of the functions $\rho$ in (2.24) on the Fourier variables $\beta_{a}, \gamma$, provided the latter are finite. This is the situation one encounters when calculating the PT distribution. Here the $\beta_{a}, \gamma$-integrals are well convergent so that $\beta_{a}, \gamma=\mathcal{O}(1)$ and we can simplify the $\beta_{a}, \gamma$-dependence by expanding the logarithmic functions $R_{a}$ in (2.23) to SL accuracy as

$$
r\left(\rho, Q_{a}^{\mathrm{PT}}\right)=r\left(\bar{\nu}, Q_{a}^{\mathrm{PT}}\right)+\ln \left(\frac{\rho}{\bar{\nu}}\right) r^{\prime}\left(\nu, Q_{a}^{\mathrm{PT}}\right)+\mathcal{O}\left(\alpha_{\mathrm{s}}\right),
$$

where

$$
r^{\prime}(\nu, Q)=\nu \partial_{\nu} r(\nu, Q)=\frac{2 \alpha_{\mathrm{s}}(1 / \nu)}{\pi} \ln (\nu Q) .
$$

Since $r^{\prime}$ is a SL function, we do not need to care about the precise expression for the hard scale in (2.28) and about the difference between $\nu, \bar{\nu}, \nu / 2$ or alike. 
Physically, the dominant integration region $\beta_{a}, \gamma=\mathcal{O}(1)$ corresponds to the situation when the hard parton recoils and the secondary parton momenta are of the same order:

$$
\left|q_{a x}\right| \sim\left|k_{i x}\right| \sim K_{\text {out }}
$$

Using (2.27) we derive then, with SL accuracy,

$$
\mathcal{R}^{\mathrm{PT}}(\nu, \beta, \gamma) \simeq \sum_{a} R_{a}(\bar{\nu})+S(\nu, \beta, \gamma)
$$

where $S$ is the SL function

$$
\begin{aligned}
S=R_{1}^{\prime}(\nu) \int_{0}^{\infty} \frac{d y}{\pi\left(1+y^{2}\right)} & \ln \sqrt{\left((1-i \gamma y)^{2}+\beta_{12}^{2}\right)\left((1+i \gamma y)^{2}+\beta_{13}^{2}\right)} \\
& +\sum_{a=2,3} R_{a}^{\prime}(\nu) \ln \sqrt{1+\beta_{a}^{2}}
\end{aligned}
$$

and $R_{a}^{\prime}(\nu)=C_{a} r^{\prime}(\nu, Q)$. In the expression (2.29), the geometry of the event enters through the DL radiators $R_{a}(\bar{\nu})$ while the SL function $S$ contains the Fourier variable dependence and embodies hard parton recoil effects. Through the colour factors in (2.30), the recoil effects depend on the specific configuration $\delta$.

Let us stress that the approximate expression (2.29) can be used only if the $\beta_{a}, \gamma$-integrals converge, which is the case of the PT distribution. In the evaluation of the NP correction instead, one encounters poorly convergent (logarithmic) $\beta_{a}, \gamma_{-}$ integrals and we cannot use the approximation (2.29) but have to employ the full expression (2.23).

\subsubsection{Recollection of the PT distribution in $K_{\text {out }}^{T}$}

Since the PT radiator is recoil-insensitive, we can freely integrate over $q_{1 y}$ and $q_{a x}$. The integrals in the Fourier variables are convergent, so that we can use the approximation (2.29) and obtain, to SL accuracy, the final result

$$
\sigma^{\mathrm{PT}}(\nu)=\int d \mu^{T} e^{-\mathcal{R}^{\mathrm{PT}}(\nu, \gamma, \beta)}=e^{-\sum R_{a}(\bar{\nu})} \mathcal{F}_{T}(\nu), \quad \mathcal{F}_{T}(\nu)=\int d \mu^{T} e^{-S(\nu, \gamma, \beta)},
$$

where $d \mu^{T}$ is the integration introduced in (2.13). Here $\mathcal{F}_{T}(\nu)$ depends on $\nu$ via the SL function $r^{\prime}(\nu, Q)$ and has been computed in [1]. To first order in $r^{\prime}$ one has

$$
\mathcal{F}_{T}(\nu)=1-\ln 2 r^{\prime}\left(2 C_{1}+C_{2}+C_{3}\right)+\mathcal{O}\left(r^{\prime 2}\right) \quad R_{a}^{\prime}=C_{a} r^{\prime}(\nu, Q) .
$$

We now recall how to compute the distribution, i.e. how to perform the $\nu$-integral in the Mellin representation (2.14). To this end we use the identity

$$
\begin{aligned}
\Sigma^{\mathrm{PT}}\left(K_{\text {out }}\right) & =\int \frac{d \nu e^{\nu K_{\text {out }}}}{2 \pi i \nu}\left\{e^{-\sum R_{a}(\bar{\nu})} \cdot \mathcal{F}_{T}(\nu)\right\} \\
& =\left.e^{-\sum R_{a}\left(\bar{K}_{\text {out }}^{-1} e^{-\partial_{z}}\right)} \cdot \mathcal{F}_{T}\left(K_{\text {out }}^{-1} e^{-\partial_{z}}\right) \frac{1}{\Gamma(1+z)}\right|_{z=0}, \quad \bar{K}_{\text {out }}=e^{-\gamma_{E}} K_{\text {out }} .
\end{aligned}
$$


The action of this differential operator on the function $\Gamma^{-1}(1+z)$ reduces, within the adopted accuracy, simply to replacing $z$ with $R_{T}^{\prime}$. The PT distribution to SL accuracy takes the form

$$
\Sigma^{\mathrm{PT}}\left(K_{\text {out }}\right) \simeq e^{-\sum_{a} R_{a}\left(\bar{K}_{\text {out }}^{-1}\right)} \cdot \frac{\mathcal{F}_{T}\left(K_{\text {out }}^{-1}\right)}{\Gamma\left(1+R_{T}^{\prime}\right)}, \quad R_{T}^{\prime} \equiv C_{T} r^{\prime}\left(K_{\text {out }}^{-1}, Q\right),
$$

with $C_{T}=2 C_{F}+N_{c}$ the total colour charge. Dependence on the geometry enters through the hard scales in the DL radiators $R_{a}\left(\bar{K}_{\text {out }}^{-1}\right)$, while the hard parton recoils build up the SL correction factor $\mathcal{F}_{T}\left(K_{\text {out }}^{-1}\right)$.

The rôle of recoil effects and the event-plane kinematics can be seen already in the first-order expansion in $\alpha_{\mathrm{s}}$. Using the expansion of $\mathcal{F}_{T}$ given in (2.32), for the differential distribution we obtain

$$
\frac{d \Sigma^{\mathrm{PT}}\left(K_{\text {out }}\right)}{d \ln K_{\text {out }}}=\frac{2 \alpha_{\mathrm{s}}}{\pi}\left\{C_{1} \ln \frac{4 Q_{1}^{\mathrm{PT}}}{K_{\text {out }}}+C_{2} \ln \frac{2 Q_{2}^{\mathrm{PT}}}{K_{\text {out }}}+C_{3} \ln \frac{2 Q_{3}^{\mathrm{PT}}}{K_{\text {out }}}\right\}+\mathcal{O}\left(\alpha_{\mathrm{s}}^{2}\right) .
$$

Gluon emission off the parton $p_{a}$ has its characteristic hard scale $Q_{a}^{\mathrm{PT}}$. The additional factors 4 and 2, that enter the hard scales, originate from the SL function $\mathcal{F}_{T}$ in (2.32) and have a simple kinematical origin. The standard definition of the event plane implies that when the secondary gluon is emitted in the right hemisphere (see Fig. 2b), all three hard partons experience equal recoils,

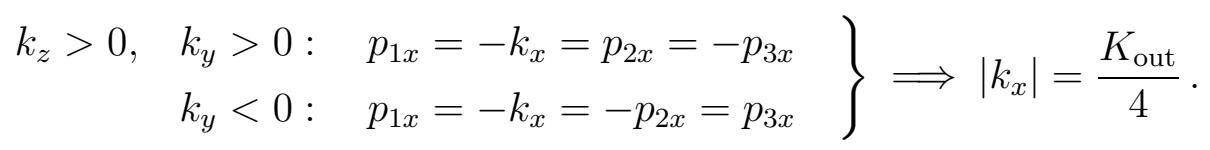

On the other hand, for the secondary gluon in the left hemisphere (see Fig. 2c and $2 \mathrm{~d}$ ), only one parton recoils against it:

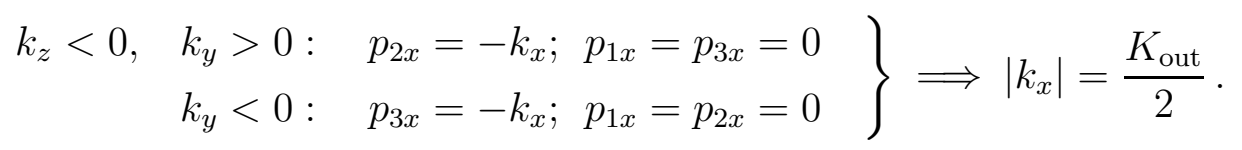

These are the factors 4 or 2 entering the scales in (2.35).

\section{$2.5 K_{\text {out }}^{R}$ case: NP radiator and recollection of PT results}

Here we describe how the results of the previous subsection change in the case of the $K_{\text {out }}^{R}$ distribution. (In this subsection the suppressed indices $R$ and $\delta$ are implied.)

\subsection{1 $K_{\text {out }}^{R}$-radiator}

As in the previous case, the radiator for the $K_{\text {out }}^{R}$ distribution has PT and NP components:

$$
\mathcal{R}\left(\nu, \beta, q_{1 x}\right)=\mathcal{R}^{\mathrm{PT}}(\nu, \beta)+\delta \mathcal{R}\left(q_{1 x}\right) .
$$




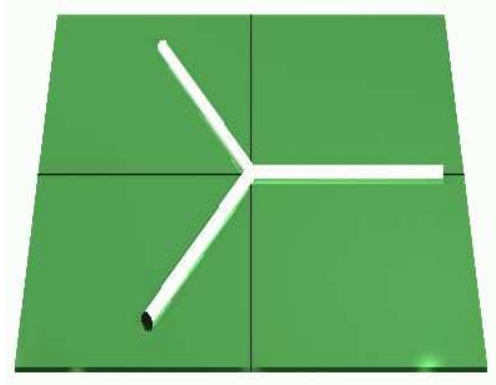

(a)

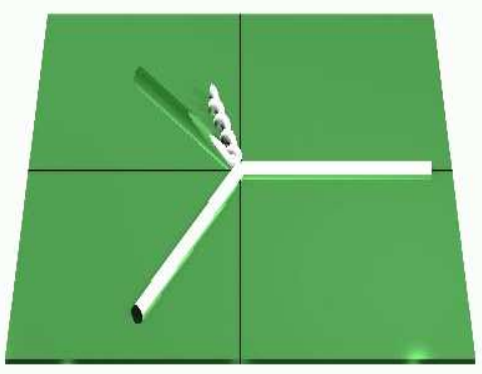

(c)

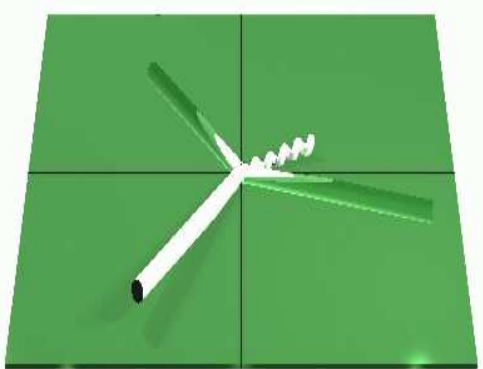

(b)

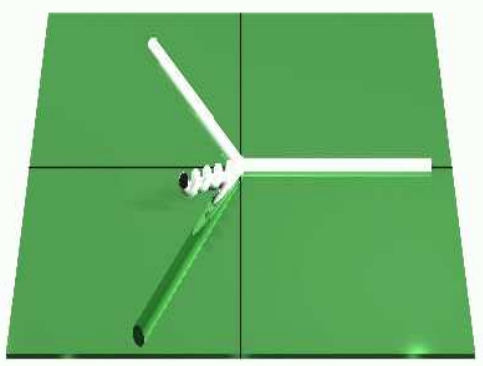

(d)

Figure 2: (a) Hard partons in a generic Born configuration. (b) Soft gluon $k$ (the short curly stick) is emitted in the right hemisphere. All three hard partons experience equal out-of-plane recoils, see (2.36). White (shadowed) partons have positive (negative) $x$ components of the momentum. (c) Soft gluon $k$ is emitted in the up-left region. According to $(2.37)$, only parton $\# 2$ recoils (shadowed; it has a negative $x$-components of the momentum). (d) The case of $k$ in the down-left region.

The PT radiator does not depend on $q_{1 x}$ while the NP correction does not depend on $\beta$.

The NP correction is described in details in Appendix B. Since the observable accumulates only particles emitted in the right hemisphere which contains only one hard parton \#1, the NP radiator can be written as

$$
\delta \mathcal{R}\left(q_{1 x}\right)=\nu \lambda^{\mathrm{NP}} C_{1} \ln \frac{Q_{1}^{\mathrm{NP}}}{\left|q_{1 x}\right|} .
$$

Subleading contributions due to radiation into the $R$ hemisphere off the $L$-hemisphere partons are embodied in the hard NP scale $Q_{1}^{\mathrm{NP}} \sim Q$. This new scale $i s$ not related to the NP hard scale (2.21) for the $K_{\text {out }}^{T}$ case. ${ }^{3}$ Contrary to the $K_{\text {out }}^{T}$-case, the hard scale in (2.32) does not have a simple geometrical interpretation. This is due to the fact that particle selection in the $R$-hemisphere is "unnatural" for the two-parton antenna dipoles that determine the structure of the accompanying soft radiation in

\footnotetext{
${ }^{3}$ Denoting, for the sake of simplicity, the hard scales for $R$ - and $T$-distributions by the same symbol does not cause confusion.
} 
the three-jet event. The procedure for computing the $R$-scale in (2.39) is described in Appendix B.5.

To evaluate the effect of the NP radiator upon the distribution we need to know the PT radiator. It has been computed in [1] (see also Appendix A) and reads

$$
\mathcal{R}^{\mathrm{PT}}(\nu \beta)=R_{1}(\rho)=C_{1} r\left(\rho, Q_{1}^{\mathrm{PT}}\right), \quad \rho=\bar{\nu} \sqrt{1+\beta^{2}},
$$

with $r$ the DL function defined in (2.25). The hard PT scale $Q_{1}^{\mathrm{PT}} \sim Q$ associated with the soft parton emission in the right region, does not coincide with the hard scale (2.26) for the PT $K_{\text {out }}^{T}$-radiator and, once again, does not have a simple geometrical meaning.

\subsubsection{Recollection of the PT distribution in $K_{\mathrm{out}}^{R}$}

The situation here is simpler than before since we have a single Fourier variable $\beta$.

Neglecting the NP radiator, the $q_{1 x}$-integral can be freely performed and one obtains

$$
\Sigma^{\mathrm{PT}}\left(K_{\text {out }}\right)=\int \frac{d \nu e^{\nu K_{\text {out }}}}{2 i \pi \nu} \sigma^{\mathrm{PT}}(\nu), \quad \sigma^{P T}(\nu)=\int_{-\infty}^{\infty} \frac{d \beta}{\pi\left(1+\beta^{2}\right)} e^{-R_{1}\left(\bar{\nu} \sqrt{1+\beta^{2}}\right)} .
$$

By using the identity

$$
\Sigma^{\mathrm{PT}}\left(K_{\text {out }}\right)=e^{-R_{1}\left(\bar{K}_{\text {out }}^{-1} e^{-\partial_{z}}\right)} \frac{f(z)}{\Gamma(1+z)}, \quad f(z)=\frac{\Gamma\left(\frac{1+z}{2}\right)}{\sqrt{\pi} \Gamma\left(1+\frac{1}{2} z\right)},
$$

we obtain the SL expression of the $K_{\text {out }}^{R}$ distribution

$$
\Sigma^{\mathrm{PT}}\left(K_{\text {out }}\right) \simeq e^{-R_{1}\left(\bar{K}_{\text {out }}^{-1}\right)} \cdot \frac{f\left(R_{1}^{\prime}\right)}{\Gamma\left(1+R_{1}^{\prime}\right)}, \quad R_{1}^{\prime} \equiv C_{1} r^{\prime}\left(K_{\text {out }}^{-1}, Q\right),
$$

with corrections beyond SL accuracy. In general we introduce the jet \#a function

$$
\mathcal{F}_{a}(\nu)=e^{R_{a}(\bar{\nu})} \int_{-\infty}^{\infty} \frac{d \beta_{a} e^{-R_{a}\left(\bar{\nu} \sqrt{1+\beta_{a}^{2}}\right)}}{\pi\left(1+\beta_{a}^{2}\right)} \simeq f\left(R_{a}^{\prime}(\nu)\right) . \quad R_{a}^{\prime}(\nu) \equiv C_{a} r^{\prime}(\nu, Q),
$$

As before, the $\mathcal{O}\left(\alpha_{\mathrm{s}}\right)$ expansion of the differential distribution shows that the SL corrections have a simple kinematical explanation. From $f\left(R_{a}^{\prime}\right) \simeq 1-\ln 2 \cdot R_{a}^{\prime}$ we obtain

$$
\frac{d \Sigma^{\mathrm{PT}}\left(K_{\mathrm{out}}\right)}{d \ln K_{\mathrm{out}}}=\frac{2 \alpha_{\mathrm{s}}}{\pi} C_{1} \ln \frac{2 Q_{1}^{\mathrm{PT}}}{K_{\mathrm{out}}}+\mathcal{O}\left(\alpha_{\mathrm{s}}^{2}\right) .
$$

The additional factor 2 in the scale has the following interpretation. When a single gluon $k$ is emitted in the $R$-hemisphere (see Fig. $2 \mathrm{~b}$ ) all four partons, as we already know, experience equal recoils, $\left|k_{x}\right|$. Counting only the $R$-hemisphere particles we have $K_{\text {out }}=2\left|k_{x}\right|$. 


\section{NP correction to the $K_{\text {out }}^{T}$ distribution and mean}

(In this section the suppressed indices $T$ and $\delta$ are implicit, unless explicitly needed.) Taking into account the NP radiator (2.20) we obtain, in the linear approximation in $1 / Q$, (see Appendix B)

$$
\begin{aligned}
\sigma(\nu) & =\int d \mu^{T} e^{-\mathcal{R}(\nu, \beta, \gamma, q)}=\int d \mu^{T} e^{-\mathcal{R}^{\mathrm{PT}}(\nu, \beta, \gamma)} \prod_{a}\left(\frac{\left|q_{a x}\right|}{Q_{a}^{\mathrm{NP}}}\right)^{\nu C_{a} \lambda^{\mathrm{NP}}} \\
& =\int d \mu^{T} e^{-\mathcal{R}^{\mathrm{PT}}(\nu, \beta, \gamma)}\left\{1-\nu \lambda^{\mathrm{NP}} \sum_{a} C_{a}\left[\ln \left(\bar{\nu} Q_{a}^{\mathrm{NP}}\right)+\tilde{\chi}_{a}(\beta, \gamma)\right]\right\} .
\end{aligned}
$$

The unity in the curly brackets gives the PT contribution, while the second term describes the leading NP correction. The functions $\tilde{\chi}_{a}$ are given in Appendix B. They depend only on the Fourier variables and are constructed with use of the function $\chi(\beta)$ :

$$
\chi(\beta)=\ln \sqrt{1+\beta^{2}}+\beta \tan ^{-1} \beta=|\beta| \frac{\pi}{2}+\chi^{\prime}(\beta), \quad \int_{-\infty}^{\infty} \frac{d \beta}{\pi\left(1+\beta^{2}\right)} \chi^{\prime}(\beta)=0,
$$

with $\chi^{\prime}(\beta)=\mathcal{O}(\ln |\beta|)$ for large $\beta$. For $a=2,3$ we have $\tilde{\chi}_{a}=\chi\left(\beta_{a}\right)$. The function $\tilde{\chi}_{1}(\beta, \gamma)$ contains the combination of $\chi\left(\beta_{12}\right)$ and $\chi\left(\beta_{13}\right)$ and is defined in (B.40).

Equation (3.2) shows that the functions $\tilde{\chi}_{a}$ linearly grow for large $\left|\beta_{a}\right|$, and this makes the structure of the NP corrections quite different from that of the PT contributions. Indeed, for the PT contribution the Fourier variable integrations in $d \mu^{T}$ are rapidly convergent, so that all soft momenta are of order $K_{\text {out }}$. For the NP corrections, due to the presence of the $\tilde{\chi}_{a}$ functions, the Fourier variable integrations in $d \mu^{T}$ become logarithmic. This implies that the phase space regions with the secondary gluon momenta much smaller than $K_{\text {out }}$ become important.

From (2.31) and (3.1) we obtain

$$
\sigma(\nu)=\sigma^{\mathrm{PT}}(\nu)-\nu \lambda^{\mathrm{NP}} \sum_{a} C_{a} f_{a}(\nu),
$$

where

$$
f_{a}(\nu)=\sigma^{\mathrm{PT}}(\nu) \cdot \ln \left(\bar{\nu} Q_{a}^{\mathrm{NP}}\right)+\tilde{f}_{a}(\nu), \quad \tilde{f}_{a}(\nu)=\int d \mu^{T} \tilde{\chi}_{a}(\beta, \gamma) e^{-\mathcal{R}^{\mathrm{PT}}(\bar{\nu}, \beta, \gamma)} .
$$

The functions $\tilde{f}_{a}$ are analyzed in Appendix C. We show that they are given by an integral similar to the one for the PT contribution to the Mellin moment, except for the presence of the $\chi$ functions in the integrand:

$$
\begin{aligned}
\sigma^{\mathrm{PT}}(\nu) & =\int_{-\infty}^{\infty} \prod_{a=1}^{3} \frac{d \beta_{a}}{\pi\left(1+\beta_{a}^{2}\right)} \mathcal{S}\left(\nu, \beta_{1}, \beta_{23}\right) e^{-R_{2}\left(\bar{\nu} \sqrt{1+\beta_{2}^{2}}\right)-R_{3}\left(\bar{\nu} \sqrt{1+\beta_{3}^{2}}\right)}, \\
\tilde{f}_{a}(\nu) & =\int_{-\infty}^{\infty} \prod_{a=1}^{3} \frac{d \beta_{a}}{\pi\left(1+\beta_{a}^{2}\right)} \mathcal{S}\left(\nu, \beta_{1}, \beta_{23}\right) e^{-R_{2}\left(\bar{\nu} \sqrt{1+\beta_{2}^{2}}\right)-R_{3}\left(\bar{\nu} \sqrt{1+\beta_{3}^{2}}\right)} \cdot \chi\left(\beta_{a}\right),
\end{aligned}
$$


where $\beta_{23}=\beta_{2}+\beta_{3}$. The factor $\mathcal{S}$ is the contribution of the parton $\# 1$ (see Appendix $A$ ) and is given by

$$
\mathcal{S}\left(\nu, \beta, \beta_{23}\right)=e^{-\mathbf{R}_{1}\left(\bar{\nu}, \beta, \beta_{T}, 0\right)}-\frac{2}{\pi} \int_{0}^{\infty} \frac{d \gamma}{\gamma} \operatorname{Im} e^{-\mathbf{R}_{1}\left(\bar{\nu}, \beta, \beta_{T}, \gamma\right)}, \quad \beta_{T} \equiv \beta+\beta_{23},
$$

with $\mathbf{R}_{1}$ defined in (2.23).

The presence of the function $\chi\left(\beta_{a}\right)$, linearly growing with $\left|\beta_{a}\right|$, in the integrand of (3.5), makes the evaluation of $\tilde{f}_{a}(\nu)$ quite involved. The large values of $\beta_{a}, \gamma$, corresponding to the recoil momenta much smaller than $K_{\text {out }}$, give the dominant contribution to $\tilde{f}_{a}$. In these circumstances we cannot use the SL expansion of the PT radiator (2.29) which simplified the calculation for finite values of the Fourier variables.

The strategy of the calculation is the following. We split $\chi\left(\beta_{a}\right)$ into two pieces (3.2). The contribution of $\chi^{\prime}\left(\beta_{a}\right)$ is evaluated by the same techniques that we used for the PT case. Indeed, since $\chi^{\prime}\left(\beta_{a}\right)$ is growing only logarithmically, the Fourier variable integrations remain convergent and we can use the SL expansion (2.29). The linear piece $\frac{\pi}{2}\left|\beta_{a}\right|$ which gives the leading contribution to $\tilde{f}_{a}(\nu)$ will be analyzed separately.

Result for $\tilde{f}_{1}$. In this case the $\beta_{1}$-integration is logarithmic while the remaining $\gamma, \beta_{2}, \beta_{3}$ integrals remain convergent. To analyze the leading piece we need to study the behaviour of $\mathcal{S}$ for large $\beta_{1}$ and finite $\gamma, \beta_{2}, \beta_{3}$. In this limit the imaginary part of $\mathbf{R}_{1}$ vanishes, and $\mathcal{S}$ reduces to the first term only, see Appendix C.1,

$$
\mathcal{S}\left(\nu, \beta_{1}, \beta_{T}\right) \simeq e^{-R_{1}\left(\rho_{1}\right)}, \quad \rho_{12}, \rho_{13} \simeq \rho_{1} \equiv \bar{\nu} \sqrt{1+\beta_{1}^{2}} .
$$

This behaviour can be simply understood by considering the event-plane kinematics (2.36), (2.37). For $\beta_{1} \rightarrow \infty$ we are forcing parton \#1 to stay in the plane by suppressing PT radiation off it. At the same time, radiation off the other two partons is not constrained, since parton \#1 does not recoil when PT emission occurs in the $L$-hemisphere. As a result, $\mathcal{S}$ turns out to be simply the Sudakov suppression factor for not having PT gluon radiation off the parton \#1.

The leading piece of $\tilde{f}_{1}$ is obtained by substituting $\chi\left(\beta_{1}\right) \rightarrow \frac{\pi}{2}\left|\beta_{1}\right|$ in $(3.5)$ and using the approximation (3.7). All $\beta_{a}$ integrals are now factorized and we obtain

$$
\begin{aligned}
\tilde{f}_{1}(\nu) & \simeq \prod_{a=2,3} \int_{-\infty}^{\infty} \frac{d \beta_{a} e^{-R_{a}\left(\bar{\nu} \sqrt{1+\beta_{a}^{2}}\right)}}{\pi\left(1+\beta_{a}^{2}\right)} \int_{\bar{\nu}}^{\infty} \frac{d \rho_{1}}{\rho_{1}} e^{-R_{1}\left(\rho_{1}\right)} \\
& =\prod_{a=1}^{3} e^{-R_{a}(\bar{\nu})} \mathcal{F}_{2}(\nu) \mathcal{F}_{3}(\nu) E_{1}(\bar{\nu})
\end{aligned}
$$

with $\mathcal{F}_{a}(\nu)$ the SL functions defined in $(2.44)$. The function $E_{1}(\bar{\nu})$ given by

$$
E_{1}(\bar{\nu})=\int_{\bar{\nu}}^{\infty} \frac{d \rho}{\rho} e^{-R_{1}(\rho)+R_{1}(\bar{\nu})}
$$


has been studied in [7]. Its properties are recalled in Appendix D.

Corrections to the leading behaviour of $\tilde{f}_{1}$ are discussed in Appendix C. The SL result reads

$$
\tilde{f}_{1}(\nu)=e^{-\sum_{a} R_{a}(\bar{\nu})}\left\{\mathcal{F}_{2}(\nu) \mathcal{F}_{3}(\nu) E_{1}(\bar{\nu})+\mathcal{C}_{1}(\nu)\right\},
$$

with $\mathcal{C}_{1}(\nu)$ a SL function which vanishes for $\nu=0$ and is proportional to $r^{\prime}(\nu, Q)$.

The function $E_{1}\left(K_{\text {out }}^{-1}\right)$ is related to the restricted average of $\ln Q_{1}^{\mathrm{NP}} /\left|q_{1 x}\right|$, under the condition $\left|q_{1 x}\right| \leq K_{\text {out }}$. To compute this average, we use the Sudakov distribution in the recoil momentum $q=\left|q_{1 x}\right|$ of parton \#1,

$$
\mathcal{D}_{1}(q)=\frac{d}{d \ln q} e^{-R_{1}\left(q^{-1}\right)}
$$

and derive

$$
\begin{aligned}
L_{1}\left(K_{\text {out }}\right) & \equiv\left\langle\ln \frac{Q_{1}^{\mathrm{NP}}}{\left|q_{1 x}\right|}\right\rangle_{\left|q_{1 x}\right| \leq K_{\text {out }}}=e^{R_{1}\left(K_{\text {out }}^{-1}\right)} \int_{0}^{K_{\text {out }}} \frac{d q}{q} \mathcal{D}_{1}(q) \ln \frac{Q_{1}^{\mathrm{NP}}}{q} \\
& =\ln \frac{Q_{1}^{\mathrm{NP}}}{K_{\text {out }}}+E_{1}\left(K_{\text {out }}^{-1}\right) .
\end{aligned}
$$

From the behaviour of the function $E_{1}$, see (D.4) and (D.5), we obtain the leading terms of $L_{1}$

$$
\begin{array}{ll}
L_{1}\left(K_{\text {out }}\right)=\ln \frac{Q_{1}^{\mathrm{NP}}}{K_{\text {out }}}\left(1+\mathcal{O}\left(\left(\alpha_{\mathrm{s}} \ln ^{2} \frac{Q}{K_{\text {out }}}\right)^{-1}\right)\right), & \alpha_{\mathrm{s}} \ln ^{2} Q / K_{\text {out }} \gg 1, \\
L_{1}\left(K_{\text {out }}\right)=\left\langle\ln \frac{Q_{1}^{\mathrm{NP}}}{\left|q_{1 x}\right|}\right\rangle\left(1+\mathcal{O}\left(\alpha_{\mathrm{s}} \ln ^{2} \frac{Q}{K_{\text {out }}}\right)\right), & \alpha_{\mathrm{s}} \ln ^{2} Q / K_{\text {out }} \ll 1 .
\end{array}
$$

The unrestricted average is given by

$$
\left\langle\ln \frac{Q_{1}^{\mathrm{NP}}}{\left|q_{1 x}\right|}\right\rangle=E_{1}\left(\frac{1}{Q_{1}^{\mathrm{NP}}}\right)=\frac{\pi}{2 \sqrt{C_{1} \alpha_{\mathrm{s}}(Q)}}-\ln \frac{Q_{1}^{\mathrm{PT}}}{Q_{1}^{\mathrm{NP}}}-\frac{\beta_{0}}{6 C_{1}}+\mathcal{O}\left(\sqrt{\alpha_{\mathrm{s}}}\right) .
$$

Up to corrections of order $\sqrt{\alpha_{\mathrm{s}}}$ it is independent of the event geometry.

The restricted average $L_{1}\left(K_{\text {out }}\right)$ is a decreasing function of $K_{\text {out }}$. It starts from $\ln Q_{1}^{\mathrm{NP}} / K_{\text {out }}$ for very small $K_{\text {out }}\left(\alpha_{\mathrm{s}} \ln ^{2} Q / K_{\text {out }} \gg 1\right)$. With $K_{\text {out }}$ increasing, it freezes at the value of the unrestricted average $E_{1}\left(1 / Q_{1}^{\mathrm{NP}}\right)$ at moderately small $K_{\text {out }}$ $\left(\alpha_{\mathrm{s}} \ln ^{2} Q / K_{\text {out }} \ll 1\right)$, up to corrections of $\mathcal{O}\left(\sqrt{\alpha_{\mathrm{s}}}\right)$.

Result for $\tilde{f}_{a}$ with $a=2,3$. Consider for example the case of $\tilde{f}_{2}$. Here the $\beta_{2}$ integration is logarithmic while the $\beta_{1}, \beta_{3}$ integrals converge. Evaluation of the leading behaviour of $\mathcal{S}$ for $\beta_{1}, \beta_{3} \ll \beta_{2}$ is subtle. In this $\beta$-region the second term of $\mathcal{S}$ in (3.6) is not negligible any more since the $\gamma$-integration turns out to be logarithmic. 
As shown in Appendix $\mathrm{C}$, the leading exponential contributions $\exp \left(-\frac{1}{2} R_{1}\right)$ in the two terms of $\mathcal{S}$ cancel, and one is left with

$$
\mathcal{S}\left(\nu, \beta, \beta_{23}\right) \simeq e^{-R_{1}\left(\bar{\nu} \sqrt{1+\beta_{2}^{2}}\right)}
$$

This behaviour has the following physical explanation. For $\beta_{2} \rightarrow \infty$ we force $p_{2}$ to stay in the plane. From (2.36) we see that in this case also $p_{1}$ has to be devoid of PT radiation, since gluon emission off parton \#1 would cause parton \#2 recoiling. Therefore, the distribution for large $\beta_{2}$ is given by the product of the two Sudakov form factors: one from parton $\# 2$, giving $\exp \left(-R_{2}\left(\bar{\nu} \sqrt{1+\beta_{2}^{2}}\right)\right)$ and the other one from the parton \#1 giving (3.15). Radiation off the parton \#3 is not constrained.

The leading piece of $\tilde{f}_{2}$ is obtained by substituting in $(3.5) \chi\left(\beta_{2}\right) \rightarrow \frac{\pi}{2}\left|\beta_{2}\right|$ and using the approximation (3.15). All $\beta_{a}$ integrals in (3.5) are now factorized. The $\beta_{1}$-integral gives 1 ; the remaining two integrals produce

$$
\begin{aligned}
\tilde{f}_{2}(\nu) & \simeq \int_{-\infty}^{\infty} \frac{d \beta_{3} e^{-R_{3}\left(\bar{\nu} \sqrt{1+\beta_{3}^{2}}\right)}}{\pi\left(1+\beta_{3}^{2}\right)} \int_{\bar{\nu}}^{\infty} \frac{d \rho}{\rho} e^{-R_{1}(\rho)} e^{-R_{2}(\rho)} \\
& =\prod_{a=1}^{3} e^{-R_{a}(\bar{\nu})} \mathcal{F}_{3}(\nu) E_{2}(\bar{\nu})
\end{aligned}
$$

where we used $\rho=\bar{\nu} \sqrt{1+\beta_{2}^{2}}$. The function $E_{2}$ is defined as

$$
E_{a}(\bar{\nu})=\int_{\bar{\nu}}^{\infty} \frac{d \rho}{\rho} e^{-R_{1}(\rho)-R_{a}(\rho)} e^{R_{1}(\bar{\nu})+R_{a}(\bar{\nu})}
$$

with $a=2$ and is analyzed in Appendix D.2. Corrections to the leading behaviour of $\tilde{f}_{2}$ are discussed in Appendix C. The answer to SL accuracy reads

$$
\tilde{f}_{2}(\nu)=e^{-\sum R_{a}(\bar{\nu})}\left\{\mathcal{F}_{3}(\nu) E_{2}(\bar{\nu})+\mathcal{C}_{2}(\nu)\right\},
$$

with $\mathcal{C}_{2}(\nu)$ a SL function which vanishes for $\nu=0$ and is proportional to $r^{\prime}(\nu, Q)$. The expression for $\tilde{f}_{3}$ is obtained from (3.18) by interchanging the labels \#2 and \#3.

The functions $E_{a}\left(K_{\text {out }}^{-1}\right)$ with $a=2,3$ can be related, as above, with the average of $\ln \left(Q_{a}^{\mathrm{NP}} /\left|q_{a x}\right|\right)$ under the condition $\left|q_{a x}\right| \leq K_{\text {out }}$ :

$$
\begin{aligned}
L_{a}\left(K_{\text {out }}\right) & \equiv\left\langle\ln \frac{Q_{a}^{\mathrm{NP}}}{\left|q_{a x}\right|}\right\rangle_{\left|q_{a x}\right| \leq K_{\text {out }}}=e^{R_{1}\left(K_{\text {out }}^{-1}\right)+R_{a}\left(K_{\text {out }}^{-1}\right)} \int_{0}^{K_{\text {out }}} \frac{d q}{q} \mathcal{D}_{a}(q) \ln \frac{Q_{a}^{\mathrm{NP}}}{q} \\
& =\ln \frac{Q_{a}^{\mathrm{NP}}}{K_{\text {out }}}+E_{a}\left(K_{\text {out }}^{-1}\right),
\end{aligned}
$$

where the PT distribution over the parton recoil $\left|q_{a x}\right|$ is given by (the logarithmic derivative of) the product of the Sudakov form factors of partons \#1 and \#a:

$$
\mathcal{D}_{a}(q)=\frac{d}{d \ln q} e^{-R_{1}\left(q^{-1}\right)-R_{a}\left(q^{-1}\right)} .
$$


From the behaviour of the functions $E_{a}$ (see (D.7) and (D.8)) we have

$$
\begin{aligned}
& L_{a}\left(K_{\text {out }}\right)=\ln \frac{Q_{a}^{\mathrm{NP}}}{K_{\text {out }}}\left(1+\mathcal{O}\left(\left(\alpha_{\mathrm{s}} \ln ^{2} \frac{Q}{K_{\text {out }}}\right)^{-1}\right)\right), \quad \alpha_{\mathrm{s}} \ln ^{2} Q / K_{\text {out }} \gg 1, \\
& L_{a}\left(K_{\text {out }}\right)=\left\langle\ln \frac{Q_{a}^{\mathrm{NP}}}{\left|q_{\text {ax }}\right|}\right\rangle\left(1+\mathcal{O}\left(\alpha_{\mathrm{s}} \ln ^{2} \frac{Q}{K_{\text {out }}}\right)\right), \quad \alpha_{\mathrm{s}} \ln ^{2} Q / K_{\text {out }} \ll 1,
\end{aligned}
$$

where the unrestricted averages now read

$$
\begin{aligned}
\left\langle\ln \frac{Q_{a}^{\mathrm{NP}}}{\left|q_{a x}\right|}\right\rangle & =E_{a}\left(\frac{1}{Q_{a}^{\mathrm{NP}}}\right) \\
& \simeq \frac{\pi}{2 \sqrt{\left(C_{1}+C_{a}\right) \alpha_{\mathrm{s}}(Q)}}-\frac{1}{C_{1}+C_{a}}\left\{C_{1} \ln \frac{Q_{1}^{\mathrm{PT}}}{Q_{a}^{\mathrm{NP}}}+C_{a} \ln \frac{Q_{a}^{\mathrm{PT}}}{Q_{a}^{\mathrm{NP}}}+\frac{\beta_{0}}{6}\right\},
\end{aligned}
$$

where we dropped corrections of absolute order $\sqrt{\alpha_{\mathrm{s}}}$ (relative corrections $\mathcal{O}\left(\alpha_{\mathrm{s}}\right)$ ). The diagonal ratios $Q_{a}^{\mathrm{PT}} / Q_{a}^{\mathrm{NP}}$ include only hard corrections to the scales. The nondiagonal ratios $Q_{1}^{\mathrm{PT}} / Q_{a}^{\mathrm{NP}}$, on the contrary, depend also on the event geometry.

\subsection{Correction to the mean $K_{\text {out }}^{T}$}

The NP correction to the mean is obtained from (2.18) and (3.3) by taking formally the limit $\nu \rightarrow 0$ of the functions $f_{a}(\nu)$, which actually means $\nu Q=\mathcal{O}(1)$. We get

$$
\left\langle K_{\text {out }}^{R}\right\rangle^{\mathrm{NP}}=\lambda^{\mathrm{NP}} \sum_{a} C_{a} f_{a}(0), \quad f_{a}(0)=\lim _{\nu \rightarrow 0}\left\{\ln \left(\bar{\nu} Q_{a}^{\mathrm{NP}}\right)+E_{a}(\bar{\nu})\right\},
$$

where we used the fact that, for $\nu=\mathcal{O}(1 / Q)$, we have $R_{a}(\bar{\nu}) \rightarrow 0, \mathcal{F}_{a}(\nu) \rightarrow 1$, $\mathcal{C}_{a}(\nu) \rightarrow 0$ and $\sigma^{\mathrm{PT}}(\nu) \rightarrow 1$. The quantity in the curly brackets is $L_{a}\left(\bar{\nu}^{-1}\right)$. We obtain, up to corrections of order $\sqrt{\alpha_{\mathrm{s}}}$,

$$
f_{a}(0) \simeq\left\langle\ln \frac{Q_{a}^{\mathrm{NP}}}{\left|q_{a x}\right|}\right\rangle
$$

with the average given in (3.14) and (3.22). The final result for the mean is then

$$
\left\langle K_{\text {out }}^{T}\right\rangle^{\mathrm{NP}}=\lambda^{\mathrm{NP}} \sum_{a} C_{a}\left\langle\ln \frac{Q_{a}^{\mathrm{NP}}}{\left|q_{a x}\right|}\right\rangle+\mathcal{O}\left(\sqrt{\alpha_{\mathrm{s}}}\right) .
$$

By using the expansions (3.14) and (3.22) we get

$$
\left\langle K_{\text {out }}^{T}\right\rangle^{\mathrm{NP}}=\lambda^{\mathrm{NP}} \frac{\pi}{2 \sqrt{\alpha_{\mathrm{s}}(Q)}}\left(\sqrt{C_{1}}+\frac{C_{2}}{\sqrt{C_{1}+C_{2}}}+\frac{C_{3}}{\sqrt{C_{1}+C_{3}}}\right)+\mathcal{O}(1) .
$$

The legitimate constant term here can be restored from (3.14) and (3.22); it contains the ratios of hard PT and NP scales and depends on the geometry of the event.

The peculiar colour structure of (3.26) shows that, due to an interplay between the PT and NP effects, the three jets do not contribute independently to the mean. 


\subsection{The distribution}

From the Mellin moment (3.3) one obtains the NP correction to the integrated distribution

$$
\Sigma\left(K_{\text {out }}\right)=\Sigma^{\mathrm{PT}}\left(K_{\text {out }}\right)+\delta \Sigma\left(K_{\text {out }}\right)
$$

where

$$
\delta \Sigma\left(K_{\text {out }}\right)=-\lambda^{\mathrm{NP}} \sum_{a} C_{a} \cdot \psi_{a}\left(K_{\text {out }}\right), \quad \psi_{a}\left(K_{\text {out }}\right)=\partial_{K_{\text {out }}} \int \frac{d \nu}{2 \pi i \nu} e^{\nu K_{\text {out }}} f_{a}(\nu),
$$

with $f_{a}(\nu)$ given in (3.4). To evaluate the Mellin integral we use the operator identity

$$
\begin{aligned}
& \psi_{a}\left(K_{\text {out }}\right)=\left.\int d \mu^{T} e^{-\mathcal{R}^{\mathrm{PT}}\left(e^{\left.-\partial_{z}, \beta, \gamma\right)}\right.}\left\{\ln Q_{a}^{\mathrm{NP}}-\partial_{z}+\tilde{\chi}_{a}(\beta, \gamma)\right\} \frac{\partial_{K_{\text {out }}} \bar{K}_{\text {out }}^{z}}{\Gamma(1+z)}\right|_{z=0}
\end{aligned}
$$

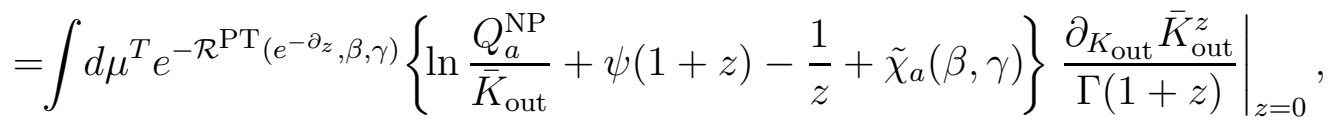

where in the first line we have replaced $\ln \nu$ with $-\partial_{z}$.

Consider the two pieces of $\psi_{a}$ given by the first two and the last two terms in the curly brackets on the second line of (3.29). The first piece can be expressed in terms of the PT distribution as follows:

$$
\psi_{a}^{(1)}\left(K_{\text {out }}\right) \simeq \Delta_{a}^{(1)} \cdot \partial_{K_{\text {out }}} \Sigma^{\mathrm{PT}}\left(K_{\text {out }}\right),
$$

where

$$
\Delta_{a}^{(1)}=\ln \frac{Q_{a}^{\mathrm{NP}}}{\bar{K}_{\mathrm{out}}}+\psi\left(1+R_{T}^{\prime}\right)=\ln \frac{Q_{a}^{\mathrm{NP}}}{K_{\text {out }}}\left(1+\mathcal{O}\left(\alpha_{\mathrm{s}}\right)\right) .
$$

Here we used the operator identity (2.33) in the form

$$
\Sigma^{\mathrm{PT}}\left(K_{\text {out }}\right)=\left.\int d \mu^{T} e^{-\mathcal{R}^{\mathrm{PT}}\left(e^{\left.-\partial_{z}, \beta, \gamma\right)}\right.} \frac{\bar{K}_{\text {out }}^{z}}{\Gamma(1+z)}\right|_{z=0},
$$

and substituted $R_{T}^{\prime}$ for $z$ with corrections of order $\mathcal{O}\left(\alpha_{\mathrm{s}}\right)$. The contribution (3.30) to the NP correction $\delta \Sigma\left(K_{\text {out }}\right)$ clearly has the form of a shift of the PT distribution.

In a similar way we calculate the second contribution to $\psi_{a}\left(K_{\text {out }}\right)$ given by the last two terms in the curly brackets of (3.29). Neglecting corrections of order $\alpha_{\mathrm{s}}$ we have $^{4}$

$$
\psi_{a}^{(2)}\left(K_{\mathrm{out}}\right) \simeq-\frac{1}{R_{T}^{\prime}} \partial_{K_{\mathrm{out}}} \Sigma^{\mathrm{PT}}\left(K_{\mathrm{out}}\right)+\frac{\partial_{K_{\mathrm{out}}} \tilde{f}_{a}\left(K_{\mathrm{out}}^{-1}\right)}{\Gamma\left(1+R_{T}^{\prime}\right)} .
$$

\footnotetext{
${ }^{4}$ The substitution of $z$ with $R_{T}^{\prime}$ is in general correct only for regular functions of $z$. Actually here we can apply the substitution for the function $1 / z$ since the singularity cancels. With more cumbersome algebra one can avoid this intermediate step altogether.
} 
Let us show that the second term with the derivative of $\tilde{f}_{a}$ generates a $1 / R_{T}^{\prime}$ contribution which cancels the singularity at $R_{T}^{\prime}=0$ of the first term in (3.33). To verify this cancellation we consider the singular pieces of $\tilde{f}_{a}$ containing the $E_{a}$ functions (see (3.8) and (3.16)).

We start from the leading piece of $\tilde{f}_{1}$ in (3.8) and take the derivative to obtain

$$
\partial_{K_{\text {out }}} \tilde{f}_{1}\left(K_{\text {out }}^{-1}\right) \simeq \frac{e^{-\sum R_{a}\left(\bar{K}_{\text {out }}^{-1}\right)}}{K_{\text {out }}} \mathcal{F}_{2}\left(K_{\text {out }}^{-1}\right) \mathcal{F}_{3}\left(K_{\text {out }}^{-1}\right)\left\{1+\left(R_{2}^{\prime}+R_{3}^{\prime}\right) E_{1}\left(\bar{K}_{\text {out }}^{-1}\right)\right\} .
$$

Using (2.34) and (2.44), we arrive at

$$
\frac{\partial_{K_{\mathrm{out}}} \tilde{f}_{1}\left(K_{\mathrm{out}}^{-1}\right)}{\Gamma\left(1+R_{T}^{\prime}\right)} \simeq \frac{\mathcal{F}_{2} \mathcal{F}_{3}}{\mathcal{F}_{T}}\left\{\frac{C_{2}+C_{3}}{C_{T}} E_{1}+\frac{1}{R_{T}^{\prime}}\right\} \cdot\left(\partial_{K_{\mathrm{out}}} \Sigma^{\mathrm{PT}}\right)
$$

with all functions in the r.h.s. evaluated at $\bar{K}_{\text {out }}^{-1}$. The second term is singular for $R_{T}^{\prime} \rightarrow 0$ and cancels with the $-1 / R_{T}^{\prime}$ term in (3.33). (Notice that $\mathcal{F}_{T}$ and $\mathcal{F}_{a}$ tend to unity in this limit.)

Similar results are obtained from the leading pieces of $\tilde{f}_{2}$ and $\tilde{f}_{3}$. Taking the derivative of (3.16) we obtain

$$
\frac{\partial_{K_{\text {out }}} \tilde{f}_{2}\left(K_{\text {out }}^{-1}\right)}{\Gamma\left(1+R_{T}^{\prime}\right)} \simeq \frac{\mathcal{F}_{3}}{\mathcal{F}_{T}}\left\{\frac{C_{3}}{C_{T}} E_{2}+\frac{1}{R_{T}^{\prime}}\right\} \cdot\left(\partial_{K_{\text {out }}} \Sigma^{\mathrm{PT}}\right),
$$

and again the $-1 / R_{T}^{\prime}$ singularity in (3.33) cancels.

The derivative of the subleading pieces $\mathcal{C}_{a}$ in the expressions (C.8) and (C.24) for $\tilde{f}_{a}$ is simpler to evaluate. We have

$$
\partial_{K_{\text {out }}}\left(\frac{\mathcal{C}_{a} e^{-\sum_{b} R_{b}}}{\Gamma\left(1+R_{T}^{\prime}\right)}\right) \simeq \frac{\mathcal{C}_{a}}{\mathcal{F}_{T}} \partial_{K_{\text {out }}} \Sigma^{\mathrm{PT}}\left(K_{\text {out }}\right)
$$

where all functions are evaluated at $\bar{K}_{\text {out }}^{-1}$ and we used again (2.34).

In conclusion, the second piece $\psi_{a}^{(2)}\left(K_{\text {out }}\right)$ of the NP corrections of the $K_{\text {out }}^{T}$ distribution can also be written as a shift of the PT distribution. Up to corrections of relative order $\alpha_{\mathrm{s}}$, we have

$$
\psi_{a}^{(2)}\left(K_{\text {out }}\right) \simeq \Delta_{a}^{(2)} \cdot \partial_{K_{\text {out }}} \Sigma^{\mathrm{PT}}\left(K_{\text {out }}\right),
$$

where

$$
\begin{aligned}
& \Delta_{1}^{(2)} \simeq \frac{C_{2}+C_{3}}{C_{T}} \frac{\mathcal{F}_{2} \mathcal{F}_{3}}{\mathcal{F}_{T}} E_{1}+\frac{\mathcal{F}_{2} \mathcal{F}_{3}-\mathcal{F}_{T}}{\mathcal{F}_{T} R_{T}^{\prime}}+\frac{\mathcal{C}_{1}}{\mathcal{F}_{T}} \\
& \Delta_{2}^{(2)} \simeq \frac{C_{3} \mathcal{F}_{3}}{C_{T} \mathcal{F}_{T}} E_{2}+\frac{\mathcal{F}_{3}-\mathcal{F}_{T}}{\mathcal{F}_{T} R_{T}^{\prime}}+\frac{\mathcal{C}_{2}}{\mathcal{F}_{T}} \\
& \Delta_{3}^{(2)} \simeq \frac{C_{2} \mathcal{F}_{2}}{C_{T} \mathcal{F}_{T}} E_{3}+\frac{\mathcal{F}_{2}-\mathcal{F}_{T}}{\mathcal{F}_{T} R_{T}^{\prime}}+\frac{\mathcal{C}_{3}}{\mathcal{F}_{T}}
\end{aligned}
$$


with all functions evaluated at $\bar{K}_{\text {out }}^{-1}$. The first terms are the leading pieces. The second terms are SL functions regular for $R_{T}^{\prime} \rightarrow 0$ (the numerators vanish when $\left.r^{\prime} \rightarrow 0\right)$. The last terms are SL corrections which vanish with $r^{\prime} \rightarrow 0$.

Summing up the two corrections (3.30) and (3.38), $\Delta_{a}=\Delta_{a}^{(1)}+\Delta_{a}^{(2)}$, we finally obtain the expression for the NP shift

$$
\Sigma\left(K_{\text {out }}\right)=\Sigma^{\mathrm{PT}}\left(K_{\text {out }}\right)+\delta K_{\text {out }} \cdot \partial_{K_{\text {out }}} \Sigma^{\mathrm{PT}}\left(K_{\text {out }}\right) \simeq \Sigma^{\mathrm{PT}}\left(K_{\text {out }}+\delta K_{\text {out }}\right),
$$

with

$$
\delta K_{\text {out }}=-\lambda^{\mathrm{NP}} \sum_{a} C_{a} \Delta_{a}\left(K_{\text {out }}\right)
$$

By expanding $\Delta_{a}^{(1)}$ and $\Delta_{a}^{(2)}$ and using the functions $L_{a}\left(K_{\text {out }}\right)$ given in $(3.12),(3.19)$ and describing the restricted averages, we arrive at

$$
\begin{aligned}
& \Delta_{1}\left(K_{\text {out }}\right) \simeq \frac{C_{2}+C_{3}}{C_{T}} \bar{L}_{1}\left(K_{\text {out }}\right)+\frac{C_{1}}{C_{T}} \ln \frac{4 Q_{1}^{\mathrm{NP}}}{K_{\text {out }}}, \\
& \Delta_{2}\left(K_{\text {out }}\right) \simeq \frac{C_{3}}{C_{T}} \bar{L}_{2}\left(K_{\text {out }}\right)+\frac{C_{1}}{C_{T}} \ln \frac{4 Q_{2}^{\mathrm{NP}}}{K_{\text {out }}}+\frac{C_{2}}{C_{T}} \ln \frac{2 Q_{2}^{\mathrm{NP}}}{K_{\text {out }}}, \\
& \Delta_{3}\left(K_{\text {out }}\right) \simeq \frac{C_{2}}{C_{T}} \bar{L}_{3}\left(K_{\text {out }}\right)+\frac{C_{1}}{C_{T}} \ln \frac{4 Q_{3}^{\mathrm{NP}}}{K_{\text {out }}}+\frac{C_{3}}{C_{T}} \ln \frac{2 Q_{3}^{\mathrm{NP}}}{K_{\text {out }}} .
\end{aligned}
$$

Here we introduced the function $\bar{L}_{a}\left(K_{\text {out }}\right)=L_{a}\left(K_{\text {out }}\right)-\gamma_{E}$ and we neglected corrections of relative order $r^{\prime}=\mathcal{O}\left(\alpha_{\mathrm{s}} \ln Q / K_{\text {out }}\right)$.

To understand the structure of the result we consider two regimes.

$\alpha_{\mathbf{s}} \ln ^{2} Q / K_{\text {out }} \gg 1$. This regime corresponds to well-developed PT cascades. From (3.13) and (3.21) we have $L_{a}\left(K_{\text {out }}\right) \sim \ln \left(Q / K_{\text {out }}\right)$, so that the colour factors in $(3.42)$ cancel and all $\Delta_{a}\left(K_{\text {out }}\right) \sim \ln \left(Q / K_{\text {out }}\right)$. For the shift we then simply have

$$
\delta K_{\mathrm{out}} \simeq-\lambda^{\mathrm{NP}} \sum_{a} C_{a}\left(\ln \frac{Q}{K_{\mathrm{out}}}+\mathcal{O}(1)\right) .
$$

In this regime multiple $\mathrm{PT}$ radiation causes all three hard partons to experience comparable recoil $\left|q_{a x}\right| \sim K_{\text {out }}$. The NP radiation off each of the partons then averages to $\ln Q_{a}^{\mathrm{NP}} /\left|q_{a x}\right| \simeq \ln Q_{a}^{\mathrm{NP}} / K_{\text {out }}$ thus producing the above result.

$\alpha_{\mathbf{s}} \ln ^{2} Q / K_{\text {out }} \ll 1$. In this regime there are only few secondary PT partons. Let us restrict ourselves to the first-order in $\alpha_{\mathrm{s}}$, that is to emission of a single PT gluon:

$$
\Sigma-\Sigma^{\mathrm{PT}} \simeq \delta K_{\text {out }} \frac{d \Sigma^{\mathrm{PT}}}{d K_{\text {out }}} \simeq \delta K_{\text {out }} \frac{R_{T}^{\prime}}{K_{\text {out }}} \Sigma^{\mathrm{PT}} \simeq \delta K_{\text {out }} \frac{R_{T}^{\prime}}{K_{\text {out }}}
$$


Observing that $R_{T}^{\prime} \propto C_{T}$, we can represent the characteristic product $\delta K_{\text {out }} R_{T}^{\prime}$ in the following equivalent form:

$$
\delta K_{\text {out }} R_{T}^{\prime}=\sum_{a=1}^{3} R_{a}^{\prime}\left(K_{\text {out }}^{-1}\right) \delta K_{\text {out }}^{(a)},
$$

where (up to $\alpha_{\mathrm{s}}$ corrections)

$$
\begin{aligned}
& \delta K_{\text {out }}^{(1)}=-\lambda^{\mathrm{NP}}\left\{C_{1} \ln \frac{4 Q_{1}^{\mathrm{NP}}}{K_{\text {out }}}+C_{2} \ln \frac{4 Q_{2}^{\mathrm{NP}}}{K_{\text {out }}}+C_{3} \ln \frac{4 Q_{3}^{\mathrm{NP}}}{K_{\text {out }}}\right\}, \\
& \delta K_{\text {out }}^{(2)}=-\lambda^{\mathrm{NP}}\left\{C_{1} \bar{L}_{1}\left(K_{\text {out }}\right)+C_{2} \ln \frac{2 Q_{2}^{\mathrm{NP}}}{K_{\text {out }}}+C_{3} \bar{L}_{3}\left(K_{\text {out }}\right)\right\}, \\
& \delta K_{\text {out }}^{(3)}=-\lambda^{\mathrm{NP}}\left\{C_{1} \bar{L}_{1}\left(K_{\text {out }}\right)+C_{2} \bar{L}_{2}\left(K_{\text {out }}\right)+C_{3} \ln \frac{2 Q_{3}^{\mathrm{NP}}}{K_{\text {out }}}\right\} .
\end{aligned}
$$

Being accompanied by the factor $R_{a}^{\prime}\left(K_{\text {out }}^{-1}\right) \propto C_{a}$ in $(3.45)$, the contribution $\delta K_{\text {out }}^{(a)}$ to the NP shift corresponds to the PT gluon emission off the parton \#a.

Thus, the shift $\delta K_{\text {out }}^{(1)}$ given by the first line in (3.46) arises when the PT gluon is emitted from $p_{1}$. In this case, as we know from (2.36), all partons undergo equal recoils, so that $\left|q_{a x}\right| \sim K_{\text {out }} / 4$. Each hard parton then contributes with $C_{a} \ln Q_{a}^{\mathrm{NP}} /\left|q_{a x}\right| \simeq C_{a} \ln \left(4 Q_{a}^{\mathrm{NP}}\right) / K_{\text {out }}$.

The shift $\delta K_{\text {out }}^{(2)}$ arises when the $\mathrm{PT}$ gluon is emitted from $p_{2}$. In this case only parton $p_{2}$ recoils with $\left|q_{2 x}\right|=K_{\text {out }} / 2$ (see (2.37)). In this situation, the $\mathrm{NP}$ gluon emitted from $p_{2}$ contributes to the shift, as before, $C_{2} \ln Q_{2}^{\mathrm{NP}} /\left|q_{2 x}\right| \simeq$ $C_{2} \ln \left(2 Q_{2}^{\mathrm{NP}}\right) / K_{\text {out }}$ (cf. the second term on the second line in (3.46)). When the NP radiation occurs off $\# 1$ and $\# 3$ the situation is entirely different. Since the partons \#1 and \#3 do not recoil against the PT gluon emission in the region \#2 (up-left quadrant), the momenta $p_{1}$ and $p_{3}$ stay in the event plane. In these circumstances the NP radiation from \#1 and \#3 would diverge if not for the high-order Sudakov form factor effects that push the partons off the plane by certain $q_{x} \neq 0$. The corresponding averages of $\ln Q_{a}^{\mathrm{NP}} /\left|q_{a x}\right|, a=1,3$ give the singular $L$-factors (3.12) and (3.19). In a similar way one can interpret various terms in $\delta K_{\text {out }}^{(3)}$.

We notice in conclusion that, using (3.40), the $K_{\text {out }}^{T}$-distribution with account of the leading NP correction can be written in the form

$$
\Sigma\left(K_{\text {out }}\right) \simeq e^{-\sum_{a} R_{a}\left(\bar{K}_{a}^{-1}\right)} \frac{\mathcal{F}_{T}\left(K_{\text {out }}^{-1}\right)}{\Gamma\left(1+R_{T}^{\prime}\right)}, \quad \bar{K}_{a}=e^{-\gamma_{E}}\left(K_{\text {out }}+\delta K_{\text {out }}^{(a)}\right) .
$$

where, up to correction of order $r^{\prime}, \delta K_{\text {out }}^{(a)}$ are given in (3.46).

\section{NP correction for the $K_{\text {out }}^{R}$ distribution and mean}

Here and in the rest of this section the indices $R$ and $\delta$ are implied. The integration 
over the recoil $q_{1 x}$ gives (see (B.39) and (B.40))

$$
\begin{aligned}
\sigma(\nu) & =\int d \mu^{R} e^{-R_{1}\left(\bar{\nu} \sqrt{1+\beta^{2}}\right)}\left(\frac{\left|q_{1 x}\right|}{Q_{1}^{\mathrm{NP}}}\right)^{\nu C_{1} \lambda^{\mathrm{NP}}} \\
& \simeq \int_{-\infty}^{\infty} \frac{d \beta e^{-R_{1}\left(\bar{\nu} \sqrt{1+\beta^{2}}\right)}}{\pi\left(1+\beta^{2}\right)}\left\{1-\nu \lambda^{\mathrm{NP}} C_{1}\left[\ln \left(\bar{\nu} Q_{1}^{\mathrm{NP}}\right)+\chi(\beta)\right]\right\},
\end{aligned}
$$

with corrections of the second order in $\lambda^{\mathrm{NP}}$. From (2.15) and (4.1) we obtain the Mellin moment

$$
\sigma(\nu) \simeq \sigma^{\mathrm{PT}}(\nu)-\nu \lambda^{\mathrm{NP}} C_{1} f_{1}(\nu), \quad f_{1}(\nu)=\sigma^{\mathrm{PT}}(\nu) \cdot \ln \left(\bar{\nu} Q_{1}^{\mathrm{NP}}\right)+\tilde{f}_{1}(\nu),
$$

where $\tilde{f}_{1}$ is given by

$$
\tilde{f}_{1}(\nu)=\int_{-\infty}^{\infty} \frac{d \beta \chi(\beta)}{\pi\left(1+\beta^{2}\right)} e^{-R_{1}\left(\bar{\nu} \sqrt{1+\beta^{2}}\right)}=e^{-R_{1}(\bar{\nu})}\left\{E_{1}(\bar{\nu})+\mathcal{C}_{1}(\nu)\right\} .
$$

This is obtained by splitting the $\chi(\beta)$ function (see $(3.2)$ ). The function $E_{1}(\bar{\nu})$ is given in (3.9) (with the PT scale $Q_{1}^{\mathrm{PT}}$ of the $K_{\text {out }}^{R}$ case), and $\mathcal{C}_{1}(\nu)$ is the SL function

$$
\mathcal{C}_{1}(\nu) \simeq \int_{-\infty}^{\infty} \frac{d \beta \chi^{\prime}(\beta)}{\pi}\left(\frac{1}{1+\beta^{2}}\right)^{1+\frac{1}{2} C_{1} r^{\prime}(\nu, Q)}
$$

Here we used the expansion $R_{1}\left(\bar{\nu} \sqrt{1+\beta^{2}}\right)-R_{1}(\bar{\nu}) \simeq \ln \sqrt{1+\beta^{2}} C_{1} r^{\prime}(\nu, Q)$ since the $\beta$-integration is fastly convergent. We now analyze the NP corrections to the mean and the distribution.

Correction to the mean $K_{\text {out }}^{R}$. The calculation is similar to that for the previous case. From (2.18), (4.2) and (4.3) we obtain

$$
\left\langle K_{\text {out }}^{R}\right\rangle_{\delta}^{\mathrm{NP}}=\lambda^{\mathrm{NP}} C_{1} f_{1}(0) \simeq \lambda^{\mathrm{NP}} C_{1}\left\langle\ln \frac{Q_{1}^{\mathrm{NP}}}{\left|q_{1 x}\right|}\right\rangle
$$

with corrections of order $\sqrt{\alpha_{\mathrm{s}}}$. The average value here is computed over the parton \#1 distribution given in (3.11) (apart from the hard scale being the one for the $K_{\text {out }}^{R}$ case).

Correction to the $K_{\text {out }}^{R}$ distribution. As before, from the Mellin moment (4.2) we obtain the NP correction to the distribution

$$
\delta \Sigma\left(K_{\text {out }}\right)=-\lambda^{\mathrm{NP}} C_{a} \psi_{1}\left(K_{\text {out }}\right), \quad \psi_{1}\left(K_{\text {out }}\right)=\partial_{K_{\text {out }}} \int \frac{d \nu}{2 \pi i \nu} e^{\nu K_{\text {out }}} f_{1}(\nu) .
$$

To evaluate $\psi_{1}$ we use the operator identity

$$
e^{-R_{1}\left(\bar{\nu} \sqrt{1+\beta^{2}}\right)}=\left.e^{-R_{1}\left(e^{-\partial_{z}}\right)}\left(\bar{\nu} \sqrt{1+\beta^{2}}\right)^{-z}\right|_{z=0} .
$$


to get

$$
\psi_{1}=\left.e^{-R_{1}\left(e^{-\partial z}\right)} \int_{-\infty}^{\infty} \frac{d \beta}{\pi}\left(\frac{1}{1+\beta^{2}}\right)^{1+\frac{z}{2}}\left(\ln \frac{Q_{1}^{\mathrm{NP}}}{\bar{K}_{\mathrm{out}}}+\psi(1+z)-\frac{1}{z}+\chi(\beta)\right) \frac{\partial_{K_{\mathrm{out}}} \bar{K}_{\text {out }}^{z}}{\Gamma(1+z)}\right|_{z=0} .
$$

The integration over $\chi(\beta)$ generates a singular $1 / z$ term:

$$
\int_{-\infty}^{\infty} \frac{d \beta \chi(\beta)}{\pi}\left(\frac{1}{1+\beta^{2}}\right)^{1+\frac{1}{2} z}=\left[\frac{1}{z}+\frac{1}{2} \psi\left(1+\frac{z}{2}\right)-\frac{1}{2} \psi\left(\frac{1+z}{2}\right)\right] \int_{-\infty}^{\infty} \frac{d \beta}{\pi}\left(\frac{1}{1+\beta^{2}}\right)^{1+\frac{1}{2} z} .
$$

This means that inside the integrand of (4.8) we can replace

$$
-\frac{1}{z}+\chi(\beta) \Rightarrow \frac{1}{2} \psi\left(1+\frac{z}{2}\right)-\frac{1}{2} \psi\left(\frac{1+z}{2}\right) .
$$

We conclude that the singular $1 / z$ term in (4.8) is cancelled by the contribution from the integral of the $\chi(\beta)$ function in (4.9).

Performing the $\beta$-integral in (4.8) we obtain the PT distribution $(z$ is substituted with $R_{1}^{\prime}$ ). As for the $K_{\text {out }}^{T}$ case, the NP correction can be expressed as a shift

$$
\Sigma\left(K_{\text {out }}\right) \simeq \Sigma^{\mathrm{PT}}\left(K_{\text {out }}+\delta K_{\text {out }}\right), \quad \delta K_{\text {out }}=-\lambda^{\mathrm{NP}} C_{1} \Delta_{1}\left(K_{\text {out }}\right),
$$

where

$$
\begin{aligned}
\Delta_{1}\left(K_{\text {out }}\right) & =\ln \frac{Q_{1}^{\mathrm{NP}}}{\bar{K}_{\text {out }}}+\psi\left(1+R_{1}^{\prime}\right)+\frac{1}{2} \psi\left(1+\frac{R_{1}^{\prime}}{2}\right)-\frac{1}{2} \psi\left(\frac{1+R_{1}^{\prime}}{2}\right) \\
& =\ln \frac{2 Q_{1}^{\mathrm{NP}}}{K_{\text {out }}}\left(1+\mathcal{O}\left(\alpha_{\mathrm{s}}\right)\right) .
\end{aligned}
$$

We see that in this case we do not have any contribution from the singular $E_{1}$ function. This is due to the fact that in (4.10) the $1 / z$ singularity was exactly cancelled by the integration over $\chi$-function. In the $K_{\text {out }}^{T}$ case the corresponding cancellation was only partial.

The physical reason for this cancellation can be understood as follows. For the $K_{\text {out }}^{R}$ distribution we are considering emissions only in the right hemisphere. In this case the parton $\# 1$ always recoils with $\left|q_{1 x}\right| \sim K_{\text {out }}$, be it the regime of welldeveloped PT cascades, $\alpha_{\mathrm{s}} \ln ^{2} Q / K_{\text {out }} \gg 1$, or the regime with few secondary partons, $\alpha_{\mathrm{s}} \ln ^{2} Q / K_{\text {out }} \ll 1$. (In this latter case even the finite factor 2 in the scale can be explained from the event plane kinematics (2.36) and Fig. 2.)

\section{Summary of relevant formulae and discussion}

In this paper we have computed the leading NP correction to the out-of-plane momentum distribution defined in (2.7) in the kinematical region (1.1). We have studied

the distributions in the variables $K_{\text {out }}^{T}$ (see (2.1)) and $K_{\text {out }}^{R}$ (see (2.2)). The NP results 
obtained here, together with the SL-resummed PT calculations carried out in [1], extend the accuracy of QCD description of CIS jet observables in $e^{+} e^{-}$annihilation processes beyond two-jet physics.

In spite of the relative complexity of three-jet ensembles, both PT and NP expressions have a clear physical interpretation based on simple QCD considerations and kinematical relations. One should expect that the present methods can be extended to other processes and CIS distributions in multi-jet environment.

We have analysed the NP corrections to the logarithmic "soft factor" $\Sigma\left(K_{\text {out }}\right)$ in the general QCD expression (2.8) based on the QCD factorization of soft radiation. The corresponding non-logarithmic coefficient function is computed in [11].

The NP corrections have been obtained by using the dispersive method of Ref. [8] for defining the running coupling and triggering the NP effects in CIS observables. The characteristic NP parameter $\lambda^{\mathrm{NP}}$ defined in (B.30) can be related, after merging of PT and NP contributions, with the integral of the running coupling over the infrared region. It is the same parameter that enters the NP corrections to distributions and means of two-jet observables such as thrust $T$, invariant jet masses $M^{2}$, the $C$-parameter and jet broadenings $B_{T}, B_{W}$. In (B.32) $\lambda^{\mathrm{NP}}$ is related to the well-known NP shift in the $1-T$ distribution, see [5].

The previous sections are full of formulae, so it may be helpful to single out the important ones and to list their main physical properties. Before turning to the NP corrections, we recall, for the sake of completeness, the key features of SL-resummed PT distribution $\Sigma^{\mathrm{PT}}\left(K_{\text {out }}\right)$.

\subsection{PT results}

We discuss first the $K_{\text {out }}^{T}$ case and then $K_{\text {out }}^{R}$.

$K_{\text {out }}^{T}$ case. The PT result is given in (2.34) with the following structures:

- the three radiators $R_{a}\left(\bar{K}_{\text {out }}^{-1}\right)$ in $(2.25)$ collect all DL $\left(\alpha_{\mathrm{s}}^{n} \ln ^{n+1} Q / K_{\text {out }}\right)$ contributions and essential SL corrections $\left(\alpha_{\mathrm{s}}^{n} \ln ^{n} Q / K_{\text {out }}\right) . R_{a}$ is the standard radiator that one encounters in the two-jet physics [2], slightly modified here by the factor 2 in the running coupling scale, which is due to the fact that the relative transverse momentum in the argument of the coupling is integrated over one (in-plane) component;

- the hard scales $Q_{a}^{\mathrm{PT}}$ are given in (2.22) and (2.26). Their precise expressions are essential only in the arguments of the DL terms (the SL correction terms do not distinguish between $Q$ and $Q_{a}^{\mathrm{PT}}$ ). It is important that these scales depend on the geometry of the event (the angles between jets);

- the additional "recoil" factor $\mathcal{F}_{T}\left(K_{\text {out }}\right.$ ) is a SL function (a function of $r^{\prime} \propto$ $\left.\alpha_{\mathrm{s}} \ln \left(Q / K_{\text {out }}\right)\right)$ given in [1] as a multiple integral over Fourier variables. This 
function depends on the configuration $\delta$ of the underlying hard process (Born parton system), as can be seen from its first-order expansion (2.32). The latter expression has a simple physical interpretation and can be easily reconstructed from event-plane kinematics with a single secondary gluon (see the discussion in subsection 2.4.2).

$K_{\text {out }}^{R}$ case. The PT result is given in (2.42) and (2.43). Here only one radiator contributes, $R_{1}\left(\bar{K}_{\text {out }}^{-1}\right)$ of the most energetic parton in the right hemisphere, so that the distribution in $K_{\text {out }}^{R}$ is sensitive to the Born configuration already at DL level. Radiation from the other two hard parton contributes only at SL level and can therefore be included in the hard momentum scale $Q_{1}^{\mathrm{PT}}$, which has been computed in [1] and does not have a simple geometrical interpretation (see the argument given in subsection 2.5.1). As in the $K_{\text {out }}^{T}$ case, the first-order expression for the distribution can be reconstructed by simply examining event-plane kinematics.

\section{$5.2 \mathrm{NP}$ result}

We have studied the NP contribution leading in $\lambda^{\mathrm{NP}}$. This approximation is valid in the region $\Lambda_{\mathrm{QCD}} \ll K_{\text {out }} \ll Q$. For values of $K_{\text {out }}$ of the order of $\Lambda_{\mathrm{QCD}}$ the simple shift approximation fails, see [9].

We start again from the case of the $K_{\text {out }}^{T}$ distribution.

$K_{\text {out }}^{T}$ case. The distribution $\Sigma\left(K_{\text {out }}\right)$ with account of the leading NP correction, is given in (3.40). The NP correction enters as a shift of the argument of the PT distribution, $\delta K_{\text {out }}$ given in (3.41). The NP gluon emitted by the hard parton $\# a$ contributes to the shift with two terms $\Delta_{a}^{(1)}\left(K_{\text {out }}\right)$ and $\Delta_{a}^{(2)}\left(K_{\text {out }}\right)$ given in (3.31) and (3.39) respectively. Corrections to these expressions are of the relative order $\alpha_{\mathrm{s}}$ and are beyond our accuracy.

The first term $\Delta_{a}^{(1)}\left(K_{\text {out }}\right)$ is additive (independent emission) since its contribution to $\delta K_{\text {out }}$ is proportional to the colour charge $C_{a}$ of the emitter. The functions $\Delta_{a}^{(1)}\left(K_{\text {out }}\right)$ behave logarithmically in $K_{\text {out }}$, with the hard NP scales $Q_{a}^{\mathrm{NP}}$ given in (2.21) and (2.22). These scales differ from the corresponding PT scales (2.26) only by constant factors $\left(\zeta^{\mathrm{NP}}\right.$ versus $\left.\zeta_{a}^{\mathrm{PT}}\right)$, which are due to small-angle radiation effects.

The second term $\Delta_{a}^{(2)}\left(K_{\text {out }}\right)$ is expressed in terms of the PT functions $\mathcal{F}_{T}\left(K_{\text {out }}^{-1}\right)$, $\mathcal{F}_{a}\left(K_{\text {out }}^{-1}\right)$ and $\mathcal{C}_{a}\left(K_{\text {out }}^{-1}\right)$, and the NP function $E_{a}\left(K_{\text {out }}^{-1}\right)$. In particular, $\mathcal{F}_{T}\left(K_{\text {out }}^{-1}\right)$ and $\mathcal{F}_{a}\left(K_{\text {out }}^{-1}\right)$ are the SL functions encountered in the PT analysis. The first is given in [1] and the second in (2.44). The SL functions $\mathcal{C}_{a}\left(K_{\text {out }}^{-1}\right)$ are defined in Appendix C. Finally, the NP functions $E_{a}\left(K_{\text {out }}^{-1}\right)$, defined in (3.9) and (3.17), are typical for observables that are uniform in the rapidity of the emitted particles. They have first emerged in the study on the NP correction to the total broadening distributions in [7].

One has the following two regimes for the shift: 
- for very small $K_{\text {out }}\left(\alpha_{\mathrm{s}} \ln ^{2} Q / K_{\text {out }} \gg 1\right)$, when the QCD cascade is welldeveloped and there are many secondary partons around, the shift $\delta K_{\text {out }}$ is dominated by $\Delta_{a}^{(1)}$, is logarithmic in $K_{\text {out }}$ and proportional to the total colour charge $C_{T}=2 C_{F}+N_{c}$ of the three-parton system:

$$
\delta K_{\mathrm{out}} \simeq-\lambda^{\mathrm{NP}} C_{T} \ln \frac{Q}{K_{\mathrm{out}}} .
$$

In this region the NP radiation effects are additive;

- in the opposite region with moderately small $K_{\text {out }}\left(\alpha_{\mathrm{s}} \ln ^{2} Q / K_{\text {out }} \ll 1\right)$, when there are few secondary partons, the shift is dominated by the contributions $\Delta_{a}^{(2)}$ that are singular in $\alpha_{\mathrm{s}}$, and the dependence on the colour charges becomes rather odd:

$$
\delta K_{\mathrm{out}} \simeq-\frac{\pi \lambda^{\mathrm{NP}}}{2 C_{T} \sqrt{\alpha_{\mathrm{s}}}}\left(\frac{C_{1}\left(C_{2}+C_{3}\right)}{\sqrt{C_{1}}}+\frac{C_{2} C_{3}}{\sqrt{C_{1}+C_{2}}}+\frac{C_{2} C_{3}}{\sqrt{C_{1}+C_{3}}}\right) .
$$

This peculiar colour structure results from an interplay between the NP and PT radiation effects. Its physical origin is discussed in detail in subsection 3.2, below (3.42).

$K_{\text {out }}^{R}$ case. The $K_{\text {out }}^{R}$ distribution, with account of the leading NP correction, is given in (4.11) with the NP shift given in (4.12). The procedure for computing the hard NP scale $Q_{1}^{\mathrm{NP}}$ is presented in Appendix B. This scale does not have a simple geometrical meaning. In agreement with the usual argument based on the event plane kinematics (see (2.36) and the discussion after (4.12)), the shift in the

$K_{\text {out }}^{R}$ distribution is purely logarithmic in $K_{\text {out }}$. Unlike the $K_{\text {out }}^{T}$ case, it does not contain singular $1 / \sqrt{\alpha_{\mathrm{s}}}$ contributions. This feature is similar to that of the single-jet broadening distribution [7].

NP corrections to the $K_{\text {out }}$ means. The results are given in (3.25) and (4.5) for the total and the right-hemisphere observables, respectively. They are expressed in terms of the unrestricted averages (3.14) and (3.22). Again, the peculiar colourcharge dependence is due to the PT-NP interplay.

\subsection{Final considerations}

To demonstrate the structure of $K_{\text {out }}$ spectra under study we show in Fig. 3 and Fig. 4 distributions generated by Herwig [13] for the three Born configurations. Notice that the difference between the distributions for each configuration is much more evident in the right case since it appears already at DL level. The corresponding numerical predictions following from the present theoretical analysis, including the relative weight of PT and NP components, will be presented elsewhere [11]. 


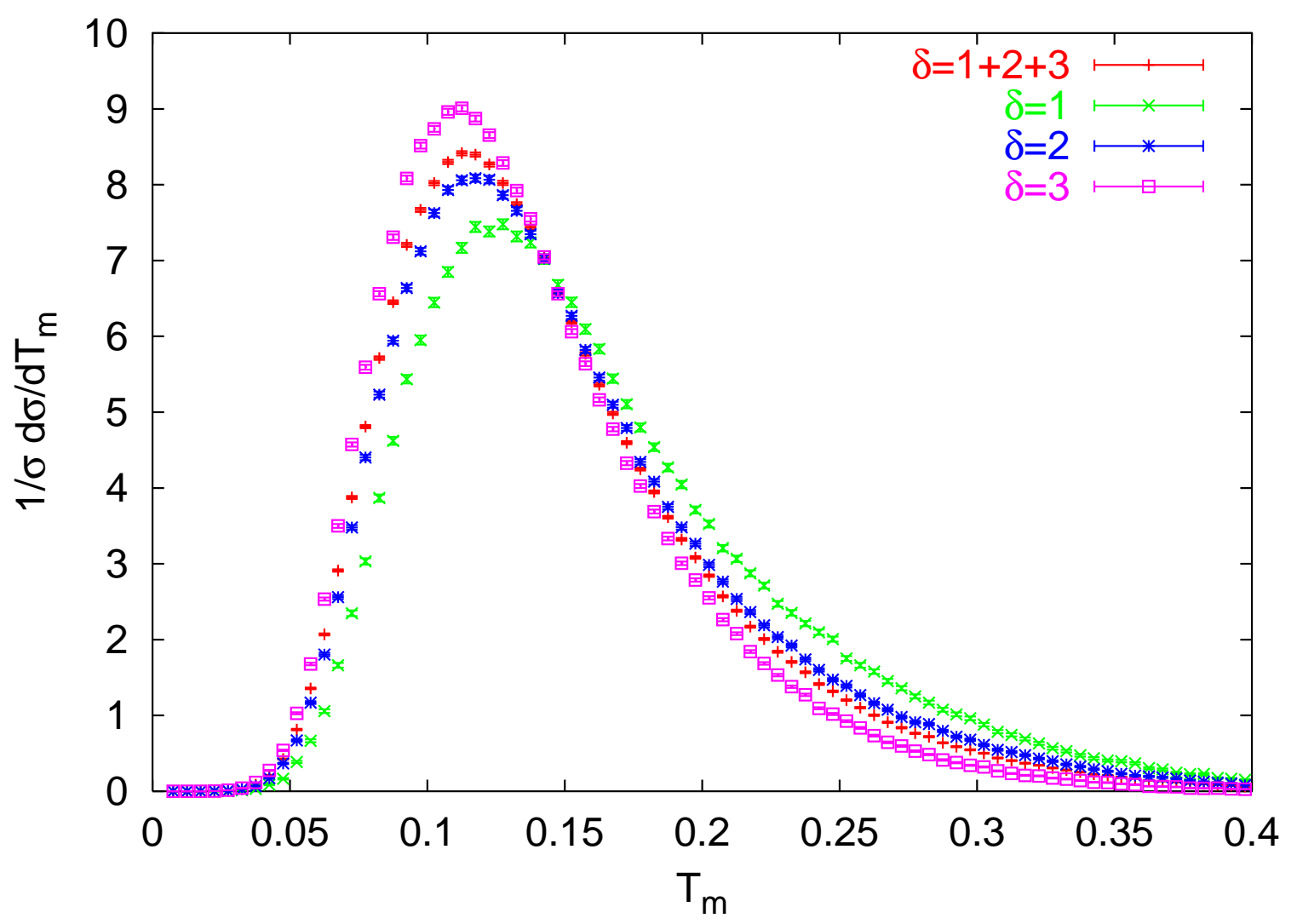

Figure 3: Herwig simulation for the total minor $\left(T_{m}=K_{\text {out }}^{T} / Q\right)$ distribution with $y_{\text {cut }}=0.1$ (Durham algorithm [14]). The picture shows the distribution for the three underlying configurations and their weighted sum.

We are intended to explore whether the approach we have developed here for the analysis of the PT and NP contributions to the $K_{\text {out }}$ distributions and means can be extended to more complicated processes and other multi-jet observables. What makes one hopeful is the observation that, in spite of technical complications, the final results, both in the PT and NP sectors, do possess a simple physical interpretation.

In particular, we plan to analyze the case with one or two jets in the initial state (lepton-hadron and hadron-hadron collisions, respectively). The resulting distribution should be sensitive to the structure of the underlying hard matrix element, as it is the case for the distributions considered in this paper. Carrying out such a programme, and comparing the QCD predictions with experiment, should provide a deeper understanding of the hadronization physics.

Acknowledgements We are grateful to Gavin Salam for helpful discussions and suggestions. 


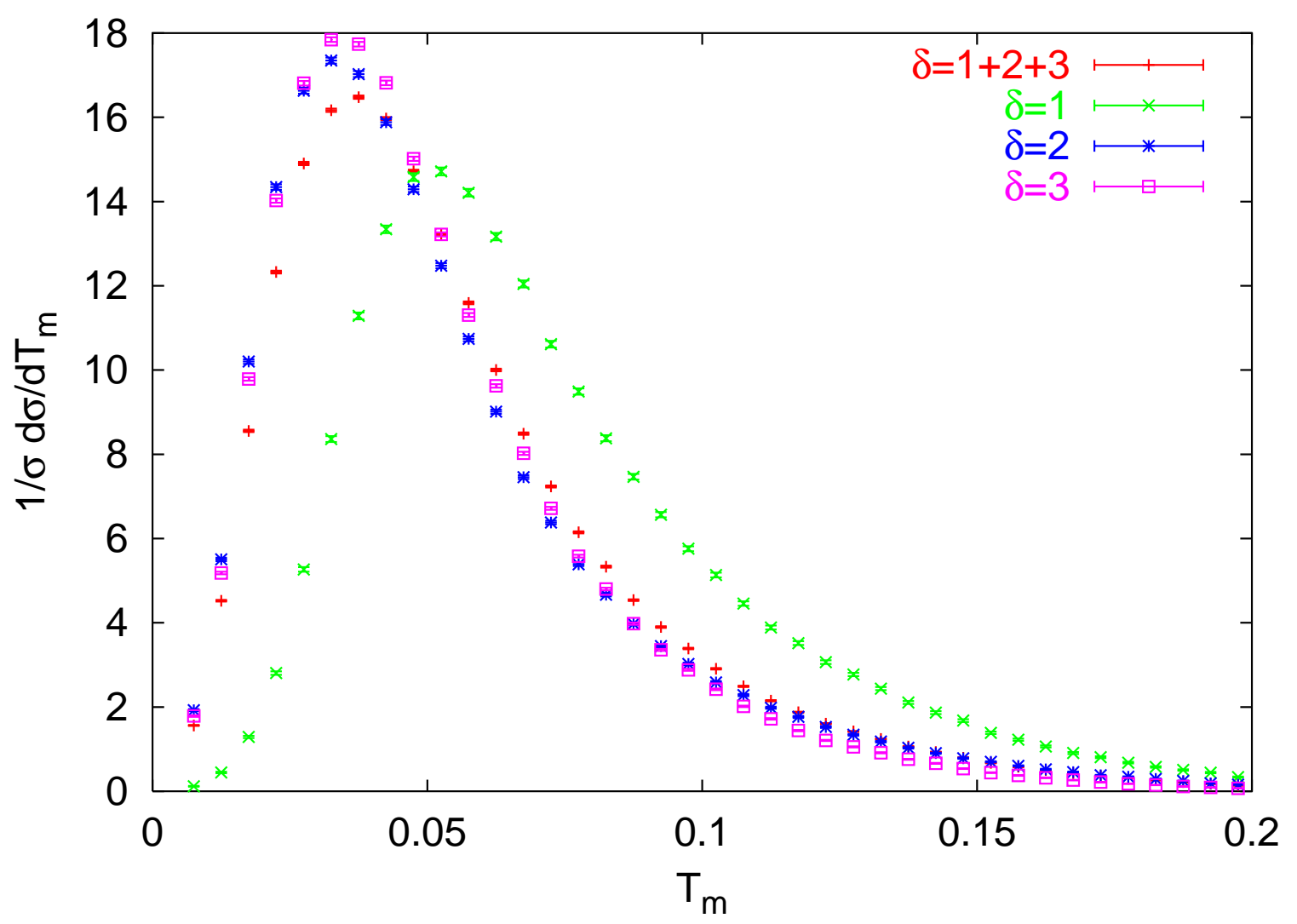

Figure 4: Herwig simulation for the narrow minor $\left(T_{m}=K_{\text {out }}^{R} / Q\right)$ distribution with $y_{c u t}=0.1$ (Durham algorithm). The picture shows the distribution for the three underlying configurations and their weighted sum.

\section{A. Soft radiation resummation and PT distributions}

The resummation of the PT contributions to the distribution $\Sigma_{\delta}^{T / R}$ to SL accuracy has been performed in [1]. To achieve such an accuracy one needs $M_{n, \delta}^{2}$ at two-loop level. The recoil momenta $q_{a}$ need to be taken into account in the kinematics, but they can be neglected in $M_{n, \delta}^{2}$. The essential point for the resummation of the soft radiation is the factorization of $M_{n, \delta}^{2}$ and of $d H_{n}^{R / T}$, the soft multi-parton distributions and the phase spaces (2.10) and (2.11). In this Appendix we recall the PT relevant points needed to analyze the NP corrections in $\Sigma\left(K_{\text {out }}\right)$ which emerge as an interplay between PT and PT contributions.

Before turning to $\Sigma^{\mathrm{PT}}\left(K_{\text {out }}\right)$ we recall the structure of the soft matrix element factorization at two-loop level. We start with the one loop expression, without virtual corrections, given by

$$
M_{n, \delta}^{2} \simeq \prod_{i=1}^{n} W_{\delta}\left(k_{i}\right),
$$

where $W_{\delta}$ is the distribution of soft gluon radiation off the hard three-parton antenna 
in the configuration $\delta$. For instance for $\delta=3$ one has

$$
\begin{aligned}
& W_{3}(k)=\frac{N_{c}}{2}\left(w_{13}+w_{23}-\frac{1}{N_{c}^{2}} w_{12}\right), \\
& w_{a b}(k)=\frac{\alpha_{\mathrm{s}}}{\pi k_{a b, t}^{2}}, \quad k_{a b, t}^{2}=\frac{2\left(p_{a} k\right)\left(k p_{b}\right)}{p_{a} p_{b}},
\end{aligned}
$$

with $k_{a b, t}$ the invariant transverse momentum of $k$ with respect to the $a b$-dipole. In general we can write

$$
W_{\delta}(k)=\sum_{a<b} C_{a b}^{(\delta)} w_{a b}(k), \quad C_{a b}^{(a)}=C_{a b}^{(b)}=\frac{N_{c}}{2}, \quad C_{a b}^{(c)}=\frac{-1}{2 N_{c}}, \quad c \neq a, b .
$$

This expression can be generalized at two-loop by considering the emission of a pair of soft partons from the three-jet configuration [12]. The two-soft emission distribution has the same form as (A.2) and this justifies the exponentiation of the two-loop soft emission distribution. As shown in [5], the two loop corrections to the soft matrix element enter at PT level simply by properly setting the argument of the running coupling in (A.2) at the dipole invariant transverse momentum. Since for the PT analysis at SL accuracy we can neglect the recoil in the soft matrix element, we set $p_{a}=P_{a}$ in $w_{a b}$.

The factorization of the phase space $d H_{n}^{T / R}$ is achieved by using the Fourier and Mellin representations for the delta and theta functions in (2.10) or (2.11). Each soft parton $k_{i}$ enters with a source $u\left(k_{i}\right)$ which, at PT level, must satisfy the condition $u\left(k_{i}\right) \rightarrow 1$ for $k_{i}$ collinear to $P_{a}$. In the next two sections we recall the form of

the sources, the radiators and the PT distributions for the $K_{\text {out }}^{T}$ and $K_{\text {out }}^{R}$ cases in succession.

\section{A.1 $K_{\text {out }}^{T}$ case: source and PT radiator}

We use

$$
\begin{aligned}
\vartheta\left(K_{\text {out }}-\sum_{a=1}^{3}\left|q_{a x}\right|-\sum_{i=1}^{n}\left|k_{i x}\right|\right) & =\int \frac{d \nu e^{\nu K_{\text {out }}}}{2 \pi i \nu} \prod_{a=1}^{3} e^{-\nu\left|q_{a x}\right|} \prod_{i} e^{-\nu\left|k_{i x}\right|}, \\
\delta^{2}\left(\vec{q}_{1}+\sum_{R} \vec{k}_{i t}\right) & =\int \frac{\nu^{2} d^{2} b_{1} e^{i \nu \overrightarrow{b_{1} \cdot \vec{q}_{1}}}}{(2 \pi)^{2}} \prod_{R} e^{i \nu \overrightarrow{b_{1}} \cdot \vec{k}_{i t}}, \\
\delta\left(q_{2 x}+q_{1 x}^{+}+\sum_{U} k_{i x}\right) & =\int \frac{\nu d \beta_{2} e^{i \nu \beta_{2}\left(q_{2 x}+q_{1 x}^{+}\right)}}{2 \pi} \prod_{U} e^{i \nu \beta_{2} k_{i x}}, \\
\delta\left(q_{3 x}+q_{1 x}^{-}+\sum_{D} k_{i x}\right) & =\int \frac{\nu d \beta_{3} e^{i \nu \beta_{3}\left(q_{3 x}+q_{1 x}^{-}\right)}}{2 \pi} \prod_{D} e^{i \nu \beta_{3} k_{i x}},
\end{aligned}
$$

with $q_{1 x}^{ \pm} \equiv q_{1 x} \vartheta\left( \pm q_{1 y}\right)$. We recall that $R=C_{1}+C_{4}, U=C_{1}+C_{2}$ and $D=C_{3}+C_{4}$ represent the right-, up- and down-regions of the phase space with $C_{\ell}$ the regions in 
the four quadrants. Each emitted soft parton contributes with the source (for the PT case)

$$
u_{T}^{(0)}(k)=\sum_{\ell=1}^{4} \Theta_{\ell}(k) u_{\ell}^{(0)}(k)
$$

where $\Theta_{\ell}(k)$ is the support function for $k \in C_{\ell}$ and

$$
\begin{array}{lc}
u_{1}^{(0)}(k)=e^{-\nu\left\{\left|k_{x}\right|-i \beta_{12} k_{x}-i \gamma k_{y}\right\}}, & u_{4}^{(0)}(k)=e^{-\nu\left\{\left|k_{x}\right|-i \beta_{13} k_{x}-i \gamma k_{y}\right\}}, \\
u_{2}^{(0)}(k)=e^{-\nu\left\{\left|k_{x}\right|-i \beta_{2} k_{x}\right\}}, & u_{3}^{(0)}(k)=e^{-\nu\left\{\left|k_{x}\right|-i \beta_{3} k_{x}\right\}}, \\
\beta_{1}=b_{1 x}, \quad \gamma=b_{1 y}, \quad \beta_{12} \equiv \beta_{1}+\beta_{2}, \quad \beta_{13} \equiv \beta_{1}+\beta_{3} .
\end{array}
$$

The source $u_{T}^{(0)}(k)$ depends on $k_{x}$ and $k_{y}$ for $k \in C_{1}$ or $C_{4}$, and only on $k_{x}$ for $k \in C_{2}$ or $C_{3}$. We obtain (2.12) and (2.13), where

$$
\begin{aligned}
V^{T}(\gamma \beta q)= & e^{-\nu\left(\left|q_{2 x}\right|-i \beta_{2} q_{2 x}\right)} e^{-\nu\left(\left|q_{3 x}\right|-i \beta_{3} q_{3 x}\right)} e^{i \nu \gamma q_{1 y}} \\
& \times\left[\vartheta\left(q_{1 y}\right) e^{-\nu\left(\left|q_{1 x}\right|-i \beta_{12} q_{1 x}\right)}+\vartheta\left(-q_{1 y}\right) e^{-\nu\left(\left|q_{1 x}\right|-i \beta_{13} q_{1 x}\right)}\right]
\end{aligned}
$$

The source (A.5) satisfies the condition $u_{T}^{(0)}(0)=1$ and therefore constitutes a suitable source for the PT analysis at SL accuracy where recoil can be neglected.

By using the factorization of soft gluon emission (A.1) and of the phase space (2.12) we can resum (2.9) and obtain

$$
\begin{aligned}
\sum_{n} \frac{1}{n !} \int d H_{n}^{T} \prod_{i}^{n}\left[d k_{i}\right] M_{n, \delta}^{2} & \Rightarrow \sum_{n=0}^{\infty} \frac{1}{n !} \int \prod_{i}\left[d k_{i}\right] W_{\delta}\left(k_{i}\right) u_{T}^{(0)}\left(k_{i}\right) \\
& =\exp \left\{\int[d k] W_{\delta}(k) u_{T}^{(0)}(k)\right\}, \quad[d k]=\frac{d^{3} k}{\pi \omega}
\end{aligned}
$$

where, in the r.h.s we removed the integration $d \mu^{T}$, see (2.13).

Next we include the virtual corrections by subtracting unity to the source and obtain the PT radiator for the total distribution

$$
\int[d k] W_{\delta}(k) u_{T}^{(0)}(k) \Rightarrow-\int[d k] W_{\delta}(k)\left[1-u_{T}^{(0)}(k)\right]=-\mathcal{R}_{\delta}^{\mathrm{PT}}\left(\nu, \beta_{a}, \gamma\right) .
$$

This expression for the PT radiator of $\Sigma^{\mathrm{PT}}$

$$
\mathcal{R}_{\delta}^{\mathrm{PT}}\left(\nu, \beta_{a}, \gamma\right)=\sum_{a<b} C_{a b}^{(\delta)} r_{a b}, \quad r_{a b}=\int \frac{d^{3} k}{\pi \omega} w_{a b}(k)\left[1-u_{T}^{(0)}\left(k_{x}\right)\right]
$$

is valid at two-loop accuracy provided one uses the correct argument of $\alpha_{\mathrm{s}}$. One has

$$
w_{a b}(k)=\frac{\alpha_{\mathrm{s}}\left(k_{t, a b}^{2}\right)}{\pi k_{t, a b}^{2}},
$$


with the coupling defined in the physical scheme [10]. This radiator has been evaluated in [1] and is given in (2.23).

The Mellin moment $\sigma_{\delta}^{\mathrm{PT}}(\nu)$ is given by the $d \mu^{T}$ integral over the three "Sudakov form factors" associated to the three hard partons. Since the PT radiator does not depend on the recoil, we can freely integrate the function $V^{T}$ over $q_{1 y}$ and $q_{a x}$ and get

$$
\begin{aligned}
I(\gamma, \beta) & =\frac{\nu^{4}}{8} \int d q_{1 y} \prod_{a} d q_{a x} V^{T}(\gamma \beta q) \\
& =\frac{1}{\left(1+\beta_{2}^{2}\right)\left(1+\beta_{3}^{2}\right)}\left(\frac{1}{\left(1+\beta_{12}^{2}\right)(-i \gamma+\epsilon)}+\frac{1}{\left(1+\beta_{13}^{2}\right)(i \gamma+\epsilon)}\right)
\end{aligned}
$$

To obtain the Mellin moment we need to integrate over the Fourier variables. Consider the integral

$$
Z\left(\nu, \beta_{23}\right) \equiv \int_{-\infty}^{\infty} \frac{d \beta_{1}}{\pi} \int_{-\infty}^{\infty} \frac{d \gamma}{2 \pi} e^{-\mathbf{R}_{1}}\left(\frac{1}{\left(1+\beta_{12}^{2}\right)(-i \gamma+\epsilon)}+\frac{1}{\left(1+\beta_{13}^{2}\right)(i \gamma+\epsilon)}\right),
$$

with $\mathbf{R}_{1}=\mathbf{R}_{1}\left(\bar{\nu}, \beta_{12}, \beta_{13}, \gamma\right)$ given in (2.23). The symmetry of the $\beta_{a}, \gamma$-integrations implies that $Z$ is a function of the sum $\beta_{23}=\beta_{2}+\beta_{3}$. The radiator $\mathbf{R}_{1}$ has an imaginary part; however, due to the symmetry

$$
\mathbf{R}_{1}\left(\bar{\nu}, \beta_{12}, \beta_{13}, \gamma\right)=\mathbf{R}_{1}\left(\bar{\nu}, \beta_{13}, \beta_{12},-\gamma\right)=\left\{\mathbf{R}_{1}\left(\bar{\nu}, \beta_{13}, \beta_{12}, \gamma\right)\right\}^{*},
$$

and to the properties of the $\beta_{2}, \beta_{3}$-integrations, the final result is real and reads

$$
Z\left(\nu, \beta_{23}\right)=\int_{-\infty}^{\infty} \frac{d \beta}{\pi\left(1+\beta^{2}\right)} \mathcal{S}\left(\nu, \beta, \beta_{23}\right),
$$

with $\mathcal{S}$ given in (3.6). The real and imaginary parts of $\mathbf{R}_{1}$ are given, respectively, by

$$
\begin{aligned}
& \mathbf{R}_{1}^{(r)}\left(\bar{\nu}, \beta, \beta^{\prime}, \gamma\right)=\frac{1}{2} R_{1}(\bar{\mu})+\frac{1}{2} R_{1}\left(\bar{\mu}^{\prime}\right), \\
& \mathbf{R}_{1}^{(i)}\left(\bar{\nu}, \beta, \beta^{\prime}, \gamma\right)=R_{1}^{\prime}(\bar{\mu}) B(\beta, \gamma)-R_{1}^{\prime}\left(\bar{\mu}^{\prime}\right) B\left(\beta^{\prime}, \gamma\right),
\end{aligned}
$$

where

$$
\bar{\mu} \equiv \bar{\nu} \sqrt{(1+\gamma)^{2}+\beta^{2}}, \quad \bar{\mu}^{\prime} \equiv \bar{\nu} \sqrt{(1+\gamma)^{2}+{\beta^{\prime}}^{2}},
$$

and where the function $B(\beta, \gamma)$ is given by

$$
\begin{aligned}
B(\beta, \gamma) & \equiv \int_{0}^{\infty} \frac{d x}{2 \pi\left(1-x^{2}\right)} \ln \frac{(1+x \gamma)^{2}+\beta^{2}}{(1+\gamma)^{2}+\beta^{2}} \\
& =\int_{0}^{1} \frac{d x}{2 \pi\left(1-x^{2}\right)} \ln x^{2} \frac{(1+x \gamma)^{2}+\beta^{2}}{(x+\gamma)^{2}+x^{2} \beta^{2}}
\end{aligned}
$$

This function has the following limits

$$
B(\beta, \gamma) \rightarrow 0 \text { for } 1, \gamma \ll \beta, \quad B(\beta, \gamma) \rightarrow-\frac{\pi}{4} \text { for } 1, \beta \ll \gamma .
$$




\section{A.2 $K_{\text {out }}^{R}$ case: source and PT radiator}

We factorize the phase space by using Mellin and Fourier representation and we obtain (2.12) and (2.13) with

$$
V^{R}\left(\beta, q_{1 x}\right)=e^{-\nu\left(\left|q_{1 x}\right|-i q_{1 x} \beta\right)},
$$

and the source for the soft parton emission

$$
u_{R}^{(0)}\left(k_{x}\right)=u^{(0)}(k) \Theta_{R}(k)+\Theta_{L}(k), \quad u^{(0)}(k) \equiv e^{-\nu\left(\left|k_{x}\right|-i \beta k_{x}\right)},
$$

where $\Theta_{R / L}(k)$ is the support function for $k \in R / L$. This source satisfies the condition $u^{(0)}(0)=1$ and therefore is a suitable source for the PT analysis at SL accuracy.

Proceeding as before, to SL accuracy, the PT radiator for the $K_{\text {out }}^{R}$ distribution is given by

$$
\mathcal{R}_{\delta}^{\mathrm{PT}}=\sum_{a<b} C_{a b}^{(\delta)} r_{a b}, \quad r_{a b}=\int \frac{d^{3} k}{\pi \omega} w_{a b}(k)\left[1-u_{R}^{(0)}(k)\right],
$$

$w_{a b}(k)$ given in (A.11). To SL accuracy the $K_{\text {out }}^{R}$-radiator is given by $(2.40)$ with $Q_{1}^{\mathrm{PT}}$ computed in $[1]^{5}$.

\section{B. NP correction to the radiators}

In this appendix we compute the NP correction to the radiators for the $K_{\text {out }}^{R / T}$ distributions. We follow the method used in [5] to compute NP corrections to various collinear and infrared safe observables in two-jet events.

We start by recalling the relevant points in the calculation of the NP corrections to the radiators for the two-jet CIS observables which we need to generalize to the present analysis. We focus our attention on the broadening observable [7] which takes contributions from the transverse momentum of the emitted particles.

\section{B.1 Recollection of two-jet case}

Kinematics. One introduces two opposite Sudakov momenta $P$ and $\bar{P}$, with $P$ along the thrust axis. For a (secondary) parton $k$ of mass $m$ the Sudakov variables are given by

$$
P=\frac{Q}{2}(1,0,0,1), \quad \bar{P}=\frac{Q}{2}(1,0,0,-1), \quad k=\alpha P+\beta \bar{P}+k_{t}, \quad \alpha \beta=\frac{k_{t}^{2}+m^{2}}{Q^{2}},
$$

with $k_{t}$ orthogonal to $P$ and $\bar{P}$. The right region $R$ is defined as

$$
k \in R: \quad \alpha>\beta \Rightarrow \alpha>\frac{\sqrt{k_{t}^{2}+m^{2}}}{Q} .
$$

\footnotetext{
${ }^{5}$ This scale depends on the configuration index $\delta$. In this reference we denote this scale for the configuration $\delta$ as $Q_{\delta}^{\mathrm{PT}}$
} 
To compute NP corrections to the broadening one needs to take into account the recoil of the primary quark and antiquark momenta for which we have a similar Sudakov decomposition.

Splitting of the radiator in three pieces. The full radiator is a CIS quantity. One rearranges [5] the two-loop radiator as a sum of three CIS terms which have been called naive, inclusive and non-inclusive pieces. The naive piece is the contribution for the emission of a single massive gluon together with the corresponding virtual corrections. Its form is given by

$$
R_{\text {naive }}^{2 \text {-jet }}=\frac{C_{F}}{\pi} \int_{0}^{Q} d m^{2} \alpha_{\text {eff }}\left(m^{2}\right) \frac{-d}{d m^{2}} \int_{0}^{Q^{2}} \frac{d^{2} \kappa}{\pi\left(\kappa^{2}+m^{2}\right)} \int_{\frac{\sqrt{\kappa^{2}+m^{2}}}{Q}}^{1} \frac{d \alpha}{\alpha}[1-u(k)]
$$

where $\alpha$ is the Sudakov variable, $\vec{\kappa}$ the transverse momentum of the soft massive gluon with respect to the hard emitting parton (including the hard parton recoil). The integration is over the right hemisphere (B.2) for the massive gluon. The effective coupling $\alpha_{\text {eff }}\left(m^{2}\right)$ is given in terms of the physical coupling in [8] through a dispersive representation.

Massive source. As already stated, the source $u(k)$ takes into account the recoil of the two hard partons and is then given in term of the relative transverse momentum $\kappa$ of the massive gluon. One is free to chose any prescription to implement the mass dependence in the source, provided that $u(k)$ reduces to the massless case for $m \rightarrow 0$. Following [5], we assume that the massive gluon source is given by the expression of the massless gluon source in which we make the replacement

$$
\kappa \rightarrow \sqrt{\kappa^{2}+m^{2}}
$$

Milan factor and universality. Using this prescription for the massive source, one has that the naive piece is the only one that needs to be computed since the remaining pieces (the inclusive and the non-inclusive pieces) contribute in a universal way simply by rescaling the naive piece by a universal Milan factor.

Linearization of the sources. The leading NP correction coming from (B.3) is obtained by taking the linear contribution in $m$ and $\kappa$ of the integrand, that is the first term in the expansion of $[1-u(k)]$. This fact has important implications on which are the relevant kinematical and Fourier variables affecting the leading NP corrections. They are the variables which enter the expression of the linearization of $[1-u(k)]$ and do not vanish upon the integration over the azimuthal angle of $\vec{\kappa}$ in (B.3). In the case of broadening [7] the Fourier variable $\vec{b}$, conjugated to the momentum $\vec{\kappa}$ does not enter the leading NP correction to the radiator. Its linear contribution to $[1-u(k)]$ is proportional to $\vec{b} \cdot \vec{\kappa}$ which vanishes upon azimuthal integration. 


\section{B.2 Sudakov variables and soft distribution for the $a b$-dipole}

We now return to our three-jet case and we start to discuss the kinematical variables. The radiator is given by the sum of three dipole contributions $r_{a b}$ (for the PT case see for instance (A.10)). To treat each dipole contribution $r_{a b}$ by the same method used in the two-jet case for (B.3) one needs to perform a Lorentz transformation. Consider the $e^{+} e^{-}$laboratory system defined by the Sudakov variables introduced in (B.1) with $P$ along the thrust axis. In this system the two massless partons $P_{a}, P_{b}$ initiating the $a b$-dipole emission are not in opposite directions. In order to follow the method of [5], we use the fact that the distribution $w_{a b}$ is Lorentz invariant and we analyze $r_{a b}$ in the $a b$-dipole center of mass (c.m.). There are three dipoles and then there are three different c.m. systems. Given a dipole $a b$, we introduce the $a b$-dipole c.m. and express the distribution $w_{a b}(k)$ in these variables. We then apply the two-jet case analysis to our case. We describe the new variables.

Dipole $a b$ center of mass frame. Consider the laboratory frame in which the Born momenta $P_{a}, P_{b}$ of the $a b$-dipole are given in (2.3). We go from this frame to the $a b$-dipole c.m. frame by a rotation in the $y z$-plane, a $z$-boost and again a rotation in the $y z$-plane which takes $P_{a}^{\prime}$ and $P_{b}^{\prime}$ along the $z$-axis. We denote by $P_{a}^{\prime}, P_{b}^{\prime}, p_{a}^{\prime}, p_{b}^{\prime}$ and $k_{i}^{\prime}$ the parton momenta in this frame with Sudakov components

$$
\begin{aligned}
& P_{a}^{\prime}=\frac{Q_{a b}}{2}(1,0,0,1), \quad P_{b}^{\prime}=\frac{Q_{a b}}{2}(1,0,0,-1), \quad Q_{a b}^{2}=2 P_{a} P_{b}, \\
& p_{a}^{\prime}=A_{a b}^{\prime} P_{a}^{\prime}+b_{a b}^{\prime} P_{b}^{\prime}+q_{a t}^{\prime}, \quad A_{a b}^{\prime} \simeq 1 \\
& p_{b}^{\prime}=a_{b a}^{\prime} P_{a}^{\prime}+B_{b a}^{\prime} P_{b}^{\prime}+q_{b t}^{\prime}, \quad B_{b a}^{\prime} \simeq 1 \\
& k_{i}^{\prime}=\alpha_{i}^{\prime} P_{a}^{\prime}+\beta_{i}^{\prime} P_{b}^{\prime}+k_{i t}^{\prime}, \quad \alpha_{i}^{\prime} \beta_{i}^{\prime}=\frac{k_{i t}^{\prime 2}}{Q_{a b}^{2}} .
\end{aligned}
$$

The transverse components are orthogonal to $P_{a}^{\prime}$ and $P_{b}^{\prime}$. All Sudakov variables are soft except for $A_{a b}^{\prime}$ and $B_{b a}^{\prime}$ which are close to one. The $x$-components are not affected by the Lorentz transformation which is in the $y z$-plane, so that the components entering our observable $K_{\text {out }}$ are the same in both frames

$$
q_{a x}^{\prime}=q_{a x}, \quad q_{b x}^{\prime}=q_{b x}, \quad k_{i x}^{\prime}=k_{i x} .
$$

We denote by $F_{a b}$ the forward region of secondary parton \# $i$ in the $a b$-dipole c.m.

$$
k_{i} \in F_{a b}: \quad \quad \alpha_{i}^{\prime}>\beta_{i}^{\prime} \Rightarrow \alpha_{i}^{\prime}>\frac{k_{i t}^{\prime}}{Q_{a b}} .
$$

The Sudakov variables (B.5) are different for different dipoles. We do not need to add an index to specify the dipole since these variables will be used only in the calculation of the specific $a b$-radiator. 
Soft distribution in the $a b$-dipole c.m. The (Lorentz invariant) $a b$-dipole soft distribution $w_{a b}$ is given in terms of $k_{a b, t}$, the "relative transverse momentum in the $a b$-dipole frame". By using the Sudakov variables (B.5) in the ab-dipole c.m. frame we have

$$
\frac{1}{k_{a b, t}^{2}} \equiv \frac{k_{t}^{\prime 2}}{\left(\vec{k}_{t}^{\prime}-\alpha^{\prime} \vec{q}_{a t}^{\prime}\right)^{2}\left(\vec{k}_{t}^{\prime}-\beta^{\prime} \vec{q}_{b t}^{\prime}\right)^{2}}
$$

where we have neglected the large components $A_{a b}^{\prime} \simeq B_{b a}^{\prime} \simeq 1$.

We split the dipole distribution $w_{a b}$ into two pieces:

$$
w_{a b}=w_{a b}^{a}+w_{a b}^{b}
$$

where $w_{a b}^{a}$ has support only in the forward region $F_{a b}$,

$$
w_{a b}^{a}(k) \equiv w_{a b}(k) \cdot \theta\left(\alpha^{\prime}-\beta^{\prime}\right) \simeq \frac{\alpha_{\mathrm{s}}\left(\kappa^{2}\right) \theta\left(\alpha^{\prime}-\beta^{\prime}\right)}{\pi \kappa^{2}}, \quad \vec{\kappa} \equiv \vec{k}_{t}^{\prime}-\alpha^{\prime} \vec{q}_{a t}^{\prime} .
$$

The "backward" piece $w_{a b}^{b}$ is similarly defined. Notice that $w_{a b}(k)$ is invariant, but the splitting in the two regions is performed in the $a b$-dipole c.m.

In three-jet events the soft distribution $W^{(\delta)}(k)$ is given by a sum of dipole soft distributions $w_{a b}(k)$, see (A.3). We decompose $w_{a b}$ into the two contributions $w_{a b}^{a}$ and $w_{a b}^{b}$ as given in (B.9) and we have

$$
W^{(\delta)}(k)=\sum_{(a b)} C_{a b}^{(\delta)} w_{a b}^{a}(k),
$$

where the sum is extended to the six ordered pair $(a b)$, i.e. $a b=12,13,21,23,31,32$. Each term contributes only in the region $F_{a b}$ (recall that $w_{a b}^{a}$ has a support only in the forward region (B.7)).

\section{B.3 Source with recoil for the $K_{\text {out }}^{T}$ distribution}

As discussed above, the leading NP corrections to the radiator are obtained from the first order expansion of $\left[1-u_{T}(k)\right]$ in the momentum components. This implies that, as in the broadening case, the leading NP radiator does not depend on the four Fourier variables $\beta_{a}$ and $\gamma$. Their first order contributions in the expansion of $\left[1-u_{T}(k)\right]$ are linear in the momentum components and then vanish upon the symmetric phase space and Fourier variable integration. We have that only the Mellin variable enters the leading NP radiator in the form $\nu\left|k_{i x}\right|$. The conclusion is that in the phase space $d H_{n}^{T / R}$ in (2.10) and (2.11) we should take into account the $\mathrm{NP}$ gluon only in the theta function giving the upper bound $K_{\text {out }}^{T / R}$. On the contrary, the NP soft gluon does not contribute to the determination of the event plane. The delta functions which fix the event plane should be taken into account only in the PT contribution. 
We now analyze how one can take into account in the sources the recoil coming from the observable $K_{\text {out }}^{T}$. As in (A.8) we start from the sum

$$
\sum_{n} \frac{1}{n !} \int d H_{n}^{T} \prod_{i}\left[d k_{i}\right] M_{n, \delta}^{2}=\int \frac{d \nu e^{\nu K_{\text {out }}}}{2 \pi i \nu} \int d \mu^{T} \exp \left\{\int[d k] W_{\delta}(k) u_{T}^{(0)}(k)\right\} .
$$

Using the expression in (B.11) for the soft gluon distribution we can write

$$
\exp \left\{\int[d k] W_{\delta}(k) u_{T}^{(0)}(k)\right\}=\prod_{(a b)} \exp \left\{C_{a b}^{(\delta)} \int[d k] w_{a b}^{a}(k) u_{T}^{(0)}(k)\right\} .
$$

From (B.10) one has that $w_{a b}^{a}(k)$ is collinear singular for

$$
\vec{\kappa}=\vec{k}_{t}^{\prime}-\alpha^{\prime} \vec{q}_{a t}^{\prime} \rightarrow 0, \quad \Rightarrow \quad k_{x}-\alpha^{\prime} q_{a x} \rightarrow 0
$$

(with the prime variables defined in (B.5), the $a b$-dipole c.m.). From the form (A.5) of these source we have $u_{T}^{(0)}(k) \neq 1$ in the limit (B.14). We then need to construct a new source $u_{T}(k)$ which, in this limit, satisfies the condition $\left[1-u_{T}(k)\right] \rightarrow 0$. This new source must include the hard parton recoils. Actually we need to consider the recoil only for the contribution to the source associated to the theta function fixing the observable $K_{\text {out }}^{T}$. To do this we start by expanding the functional in (B.13)

$$
\exp \left\{\int[d k] W_{\delta}(k) u_{T}^{(0)}(k)\right\}=\prod_{(a b)} \sum_{n_{a b}} \frac{1}{n_{a b} !} \prod_{i=1}^{n_{a b}} C_{a b}^{(\delta)} \int\left[d k_{i}\right] w_{a b}^{a}\left(k_{i}\right) u_{T}^{(0)}\left(k_{i}\right)
$$

Consider a given term in this expansion. For any given ordered pair $(a b)$ there are $n_{a b}$ soft gluons which are associated to the distribution $w_{a b}^{a}$ and are then emitted in the region $F_{a b}$. For this term in the expansion (B.15), the corresponding theta function fixing the observable $K_{\text {out }}^{T}$ gives

$$
K_{\mathrm{out}}=\sum_{a}\left|q_{a x}\right|+\sum_{i}\left|k_{i x}\right|=\sum_{a}\left|q_{a x}\right|+\sum_{(a b)}\left\{\sum_{i \in F_{a b}}\left|k_{i x}\right|\right\} .
$$

Recall that the $x$-components are the same in both the laboratory and the $a b$-dipole c.m. frames. We then introduce the subtractions

$$
\begin{aligned}
& K_{\text {out }} \simeq \sum_{a}\left|\bar{q}_{a x}\right|+\sum_{(a b)}\left\{\sum_{i \in F_{a b}}\left(\left|k_{i x}\right|-\alpha_{i}^{\prime}\left|\bar{q}_{a x}\right|\right)\right\}, \\
& \bar{q}_{a x} \equiv\left(1+\sum_{b \neq a} \sum_{i \in F_{a b}} \alpha_{i}^{\prime}\right) q_{a x},
\end{aligned}
$$

( $\alpha_{i}^{\prime}$ is defined in the $a b$-dipole c.m.). Here we have neglected corrections of second order in the soft parameters. 
Now we reconstruct the expansion (B.15) by using (B.17) instead of (B.16). While (B.16) gives (B.12), using (B.17) we obtain

$$
\begin{aligned}
\sum_{n} \frac{1}{n !} \int d H_{n}^{T} \prod_{i}\left[d k_{i}\right] M_{n, \delta}^{2}=\int \frac{d \nu e^{\nu K_{\text {out }}}}{2 \pi i \nu}\left\{\nu^{4} \frac{d \gamma d q_{1 y}}{2 \pi} \prod_{a=1}^{3} \frac{d \beta_{a} d \bar{q}_{a x}}{2 \pi} V(\bar{q})\right\} \\
\cdot \prod_{(a b)} \exp \left\{C_{a b}^{(\delta)} \int[d k] w_{a b}^{a}(k) u_{a b}(k)\right\},
\end{aligned}
$$

where $u_{a b}(k)$ is a source associated to the ordered pair $(a b)$ given by

$$
u_{a b}(k)=u_{T}^{(0)}(k) \cdot e^{\nu \alpha^{\prime}\left|\bar{q}_{a x}\right|}=e^{-\nu\left(\left|k_{x}\right|-\alpha^{\prime}\left|\bar{q}_{a x}\right|\right)} \cdot \hat{u}_{T}(k),
$$

where $\hat{u}_{T}(k)$ is the part of the source depending on the Fourier variables $\beta_{a}, \gamma$ (see (A.6)). The factor $e^{-\nu\left(\left|k_{x}\right|-\alpha^{\prime}\left|\bar{q}_{a x}\right|\right)}$, which is the only one relevant for the NP corrections, is equal to one at the singular point (B.14). We have then that (B.19) is the form of the source we are searching for.

The Jacobian for changing integration variables from $q_{a x}$ to $\bar{q}_{a x}$ is given by

$$
J(\alpha)=\prod_{a}\left(1+\sum_{b \neq a} \sum_{i \in F_{a b}} \alpha_{i}^{\prime}\right)^{-1} \simeq \prod_{(a b)} \prod_{i \in F_{a b}}\left(1-\alpha_{i}^{\prime}\right) .
$$

This can be taken into account as a correction to the soft distribution $w_{a b}^{a}(k) \rightarrow$ $\left(1-\alpha^{\prime}\right) w_{a b}^{a}(k)$. This correction corresponds to including the hard part of the splitting function, see [5]. Since in (B.18) the rescaled momentum $\bar{q}_{a x}$ is just an integration variable, in the following we shall rename it $q_{a x}$.

\section{B.4 NP radiator for the $K_{\text {out }}^{T}$ distribution}

The radiator for the $K_{\text {out }}^{T}$-distribution, given in terms of the dipole components (B.9), reads

$$
\mathcal{R}_{\delta}=\sum_{a<b} C_{a b}^{(\delta)} r_{a b}, \quad r_{a b}=r_{a b}^{a}+r_{b a}^{b}, \quad r_{a b}^{a} \equiv \int \frac{d^{3} k}{\pi \omega} w_{a b}^{a}(k)\left[1-u_{a b}(k)\right],
$$

where the integration region is restricted to the $F_{a b}$ region.

The distribution for the emission of two soft partons in the three-jet event (see [12] and [1]) can be represented as the sum of the two soft parton emission from the three dipoles. This fact can be used to obtain the two-loop PT radiator as sum of the two-loop radiator for each dipole. At this point we can use all the results of the two-jet analysis. At PT level this gives rise simply to the identification of the running coupling scale for each dipole radiator, the invariant dipole transverse momentum, and the scheme. For the analysis of the NP corrections we follow the two-jet case method recalled above. Introducing the coupling according to the dispersive method 
[8] we write the two-loop dipole radiator as a sum of three pieces, the naive, the inclusive and the non-inclusive pieces. The naive piece of the $a b$-dipole is given, as in (B.3), by

$$
r_{a b}^{a}=\int d m^{2} \alpha_{\mathrm{eff}}\left(m^{2}\right) \frac{-d}{d m^{2}} \int \frac{d^{2} \kappa}{\pi\left(\kappa^{2}+m^{2}\right)} \int_{\frac{\sqrt{\kappa^{2}+m^{2}}}{Q_{a b}}}^{1} \frac{d \alpha^{\prime}}{\alpha^{\prime}}\left[1-u_{a b}(k)\right],
$$

with $\alpha_{\text {eff }}\left(m^{2}\right)$ the effective coupling discussed in [8]. The integration region is restricted to the forward region $F_{a b}$ defined by $\alpha^{\prime}>k_{t}^{\prime} / Q_{a b}$. This has been replaced by $\alpha^{\prime}>\sqrt{\kappa^{2}+m^{2}} / Q_{a b}$, since, at the lower limit of $\alpha^{\prime}$ (large angle emission) the correction is of second order in the NP-parameter (see [7]).

As discussed in the Appendix B.1, the leading NP correction coming from (B.22) is obtained as follows:

- in the integrand we take the linear contribution in $\kappa, m \sim \Lambda_{\mathrm{QCD}} \ll\left|q_{a x}\right|$. Therefore, for the massless gluon source (B.19), we take the first term in the expansion

$$
\left[1-u_{a b}(k)\right]=\nu\left(\left|k_{x}\right|-\alpha^{\prime}\left|q_{a x}\right|\right)+\ldots \quad k_{x}=\kappa \cos \phi+\alpha^{\prime} q_{a x},
$$

where $\phi$ is the azimuthal angle of $\vec{\kappa}$. The neglected terms contribute to the higher order power corrections;

- for a gluon with mass $m$ we make the replacement (B.4). This ensures a simple treatment of the other (inclusive and non-inclusive) pieces of the twoloop radiator;

- we take the NP piece $\delta \alpha_{\text {eff }}\left(m^{2}\right)$ of the effective coupling.

The leading NP correction to the naive piece is then given by

$$
\begin{aligned}
& \delta r_{a b}^{a}=\int d m^{2} \frac{\delta \alpha_{\mathrm{eff}}\left(m^{2}\right)}{\pi} \frac{-d}{d m^{2}} \int \frac{d \kappa^{2}}{\kappa^{2}+m^{2}} \Omega_{a b}\left(\kappa^{2}+m^{2}\right) \\
& \Omega_{a b}\left(\kappa^{2}+m^{2}\right)=\nu \int_{-\pi}^{\pi} \frac{d \phi}{2 \pi} \int_{\frac{\sqrt{\kappa^{2}+m^{2}}}{Q_{a b}}}^{1} \frac{d \alpha^{\prime}}{\alpha^{\prime}}\left(\left|\sqrt{\kappa^{2}+m^{2}} \cos \phi+\alpha^{\prime} q_{a x}\right|-\alpha^{\prime}\left|q_{a x}\right|\right) .
\end{aligned}
$$

To evaluate this integral we introduce the rescaled variable

$$
v=\frac{\alpha^{\prime}\left|q_{a x}\right|}{\sqrt{\kappa^{2}+m^{2}}}
$$

and find

$$
\Omega_{a b}\left(\kappa^{2}+m^{2}\right)=\nu \sqrt{\kappa^{2}+m^{2}} \int_{-\pi}^{\pi} \frac{d \phi}{2 \pi} \int_{\frac{\left|q_{a x}\right|}{Q_{a b}}}^{1} \frac{d v}{v}(|\cos \phi-v|-v) .
$$


Notice that at the upper limit $\alpha^{\prime}=1$ one has $v=\frac{\left|q_{a x}\right|}{\sqrt{\kappa^{2}+m^{2}}}$ which tends to infinity in the limit we are considering. On the other hand the contribution with $v>1$ vanishes due to symmetry of $\phi$-integration. Setting $q_{1 x} \rightarrow 0$ at the $v$-lower limit, we find

$$
\Omega_{a b}\left(\kappa^{2}+m^{2}\right)=\nu c_{K_{\text {out }}} \sqrt{\kappa^{2}+m^{2}} \ln \frac{\zeta Q_{a b}}{\left|q_{a x}\right|}, \quad c_{K_{\text {out }}}=\frac{2}{\pi},
$$

with $c_{K_{\text {out }}}$ the characteristic number for the $K_{\text {out }}$ observable and $\zeta$, which takes into account the small-angle contribution, is given by

$$
\ln \zeta=\frac{\pi}{2} \int_{-\pi}^{\pi} \frac{d \phi}{2 \pi} \int_{0}^{1} \frac{d v}{v}\{|\cos \phi-v|-v-|\cos \phi|\}=\ln 2-2 .
$$

We get

$$
\delta r_{a b}^{a}\left(q_{a x}\right)=\nu \lambda^{\mathrm{NP}} \ln \frac{\zeta Q_{a b}}{\left|q_{a x}\right|}, \quad \lambda^{\mathrm{NP}}=c_{K_{\mathrm{out}}} \int \frac{d m^{2}}{m^{2}} \frac{\delta \alpha_{\mathrm{eff}}(m)}{\pi} m .
$$

For the "inclusive" and "non-inclusive" pieces the analysis is the standard one and we have that the full NP correction is given by (B.28) by rescaling the NP-parameter by the Milan factor $\mathcal{M}$.

The NP parameter $\lambda^{\mathrm{NP}}$ has dimension of a mass, and this implies that the NP correction is proportional to $1 / Q$. It can be expressed in terms of the integral of the running coupling over the infrared region

$$
\alpha_{0}\left(\mu_{I}\right)=\int_{0}^{\mu_{I}} \frac{d k}{\mu_{I}} \alpha_{\mathrm{s}}(k)
$$

This parameter $\alpha_{0}\left(\mu_{I}\right)$ is the same entering the two-jet shape variables $1-T, C$, $B$ and $M^{2} / Q^{2}$. After merging $\mathrm{PT}$ and NP contributions to the observable in a renormalon free manner, one has that the distribution is independent of $\mu_{I}$ and one obtains

$$
\lambda^{\mathrm{NP}} \equiv c_{K_{\mathrm{out}}} \mathcal{M} \frac{4}{\pi^{2}} \mu_{I}\left\{\alpha_{0}\left(\mu_{I}\right)-\bar{\alpha}_{\mathrm{s}}-\beta_{0} \frac{\bar{\alpha}_{\mathrm{s}}^{2}}{2 \pi}\left(\ln \frac{Q}{\mu_{I}}+\frac{K}{\beta_{0}}+1\right)\right\}, \quad \bar{\alpha}_{\mathrm{s}} \equiv \alpha_{\overline{\mathrm{MS}}}(Q) .
$$

The term proportional to $K$ accounts for mismatch between the $\overline{\mathrm{MS}}$ and the physical scheme [10] and is given by

$$
K \equiv C_{A}\left(\frac{67}{18}-\frac{\pi^{2}}{6}\right)-\frac{5}{9} n_{f}, \quad \beta_{0}=\frac{11 N_{c}}{3}-\frac{2 n_{f}}{3} .
$$

It may be useful to connect this parameter entering the shift of the $K_{\text {out }}$ distributions to the analogous one for the thrust distribution. Defining $\tau=1-T$, we have

$$
\frac{d \sigma}{d \tau}(\tau)=\frac{d \sigma^{\mathrm{PT}}}{d \tau}\left(\tau-\Delta_{\tau}\right), \quad \Delta_{\tau}=C_{F} \frac{c_{\tau} \lambda^{\mathrm{NP}}}{c_{K_{\mathrm{out}}}}
$$


where $C_{F}$ enters due to the fact that the two-jet system is made of a quark-antiquark pair, $c_{\tau}=2$ and $c_{K_{\text {out }}}=2 / \pi$ are the characteristic numbers associated to the phase space integration of the relative observables.

We can now reconstruct the NP correction to the full radiator in (B.21) and we find

$$
\begin{aligned}
& \delta \mathcal{R}_{3}(q)=\nu \lambda^{\mathrm{NP}}\left(C_{F} \ln \frac{\zeta Q_{12}}{\left|q_{1 x}\right|}+C_{F} \ln \frac{\zeta Q_{12}}{\left|q_{2 x}\right|}+C_{A} \ln \frac{\zeta Q_{23} Q_{13}}{Q_{12}\left|q_{3 x}\right|}\right) \\
& \delta \mathcal{R}_{2}(q)=\nu \lambda^{\mathrm{NP}}\left(C_{F} \ln \frac{\zeta Q_{13}}{\left|q_{1 x}\right|}+C_{F} \ln \frac{\zeta Q_{13}}{\left|q_{3 x}\right|}+C_{A} \ln \frac{\zeta Q_{23} Q_{12}}{Q_{13}\left|q_{2 x}\right|}\right) \\
& \delta \mathcal{R}_{1}(q)=\nu \lambda^{\mathrm{NP}}\left(C_{F} \ln \frac{\zeta Q_{23}}{\left|q_{3 x}\right|}+C_{F} \ln \frac{\zeta Q_{23}}{\left|q_{2 x}\right|}+C_{A} \ln \frac{\zeta Q_{12} Q_{13}}{Q_{23}\left|q_{1 x}\right|}\right)
\end{aligned}
$$

We find then that the NP scales are given by the hard scales $Q_{a}$ in $(2.22)$ rescaled by a factor $\zeta^{\mathrm{NP}}=\zeta($ see $(2.21))$.

\section{B.5 NP corrections to the $K_{\text {out }}^{R}$ distribution}

We proceed as in the previous case. Here the simplification is that we should be careful only with the collinear singularity with respect to $p_{1}$ which is contained only in the distributions $w_{1 b}^{1}(k)$.

First we consider the modification of the sources. We must include the recoil only in the source $u_{1 b}(k)$ associated to the distribution $w_{1 b}^{1}(k)$. We have

$$
u_{1 b}(k)=\Theta_{L}(k)+\Theta_{R}(k) e^{-\nu\left(\left|k_{x}\right|-\alpha^{\prime}\left|q_{1 x}\right|\right)} e^{i \beta k_{x}} .
$$

For the other sources $u_{a b}(k)$, associated to the distribution $w_{a b}^{a}(k)$ with $a \neq 1$, we do not need modifications: $u_{a b}(k)=u_{R}^{(0)}(k)$. The modification in (B.34) is accompanied by a change of variable from $q_{1 x}$ to $\bar{q}_{1 x}$ obtained by a rescaling factor similar to (B.17). However since $\bar{q}_{1 x}$ is an integration variable we shall rename it $q_{1 x}$.

Then we take the linear contribution for the massive gluon source

$$
\begin{aligned}
& {\left[1-u_{1 b}(k)\right] \quad \Rightarrow \quad \nu\left(\left|\sqrt{\kappa^{2}+m^{2}} \cos \phi+\alpha^{\prime} q_{1 x}\right|-\alpha^{\prime}\left|q_{1 x}\right|\right) \Theta_{R}(k),} \\
& {\left[1-u_{a b}(k)\right] \Rightarrow \nu \sqrt{\kappa^{2}+m^{2}}|\cos \phi| \Theta_{R}(k), \quad a \neq 1 .}
\end{aligned}
$$

Since the distribution has a singularity for the soft gluon collinear to $p_{1}$, the NP radiator has a logarithmic behaviour in $q_{1 x}$. We have

$$
\delta \mathcal{R}_{\delta}(q)=\nu \lambda^{\mathrm{NP}} C_{1}^{(\delta)} \ln \frac{Q_{1}^{\mathrm{NP}}}{\left|q_{1 x}\right|} .
$$

The $\ln \left|q_{1 x}\right|$ part is contained in $r_{12}^{1}$ and $r_{13}^{1}$ since the corresponding distributions are collinear singular in the integration region. The remaining pieces contribute only to set the NP scale. To obtain the NP hard scale $Q_{1}^{\mathrm{NP}}$ we need to analyze the individual pieces. 
To see the singular behaviour in (B.36) consider the NP correction to $r_{1 b}^{1}$. At two loop, the naive term of $r_{1 b}^{1}$ is given in terms of the effective coupling as follows

$$
r_{1 b}^{1}=\int d m^{2} \alpha_{\mathrm{eff}}\left(m^{2}\right) \frac{-d}{d m^{2}} \int \frac{d^{2} \kappa}{\pi\left(\kappa^{2}+m^{2}\right)} \int_{\frac{\sqrt{\kappa^{2}+m^{2}}}{Q_{1 b}}}^{1} \frac{d \alpha^{\prime}}{\alpha^{\prime}}\left[1-u_{1 b}(k)\right] .
$$

Taking the linear part of the source and NP part of the effective coupling, we have

$$
\begin{aligned}
& \delta r_{1 b}^{1}=\int d m^{2} \delta \alpha_{\mathrm{eff}}\left(m^{2}\right) \frac{-d}{d m^{2}} \int \frac{d \kappa^{2}}{\kappa^{2}+m^{2}} \Omega_{1 b}\left(\kappa^{2}+m^{2}\right), \\
& \Omega_{1 b}\left(\kappa^{2}+m^{2}\right)=\nu \sqrt{\kappa^{2}+m^{2}} \int_{-\pi}^{\pi} \frac{d \phi}{2 \pi} \int_{\frac{\left|q_{a x}\right|}{Q_{1 a}}}^{1} \frac{d v}{v}(|\cos \phi-v|-v) \Theta_{R}(k),
\end{aligned}
$$

where we need to express the condition in $\Theta_{R}$ in terms of the variables in the $1 b$-dipole c.m. system (see (B.5)).

\section{B.6 Recoil integration over the NP radiator}

For the $K_{\text {out }}^{T}$ case we have

$$
\begin{aligned}
& \frac{\nu^{4}}{8} \int d q_{1 y} \prod_{a} d q_{a x} V^{T}(\gamma \beta q) \prod_{a}\left(\frac{\left|q_{a x}\right|}{Q_{a}^{\mathrm{NP}}}\right)^{\nu C_{a} \lambda^{\mathrm{NP}}} \\
& \simeq I(\beta, \gamma)\left\{1-\nu \lambda^{\mathrm{NP}} \sum_{a} C_{a}\left[\ln \left(\bar{\nu} Q_{a}^{\mathrm{NP}}\right)+\tilde{\chi}_{a}(\beta, \gamma)\right]\right\},
\end{aligned}
$$

with $V^{T}$ and $I(\beta, \gamma)$ given in (A.7) and (A.12) respectively, $\tilde{\chi}_{2} \equiv \chi\left(\beta_{2}\right), \tilde{\chi}_{3} \equiv \chi\left(\beta_{3}\right)$ (see $(3.2))$ and

$$
I(\beta, \gamma) \tilde{\chi}_{1} \equiv \frac{1}{\left(1+\beta_{2}^{2}\right)\left(1+\beta_{3}^{2}\right)}\left(\frac{\chi\left(\beta_{12}\right)}{\left(1+\beta_{12}^{2}\right)(-i \gamma+\epsilon)}+\frac{\chi\left(\beta_{13}\right)}{\left(1+\beta_{13}^{2}\right)(i \gamma+\epsilon)}\right) .
$$

For the $K_{\text {out }}^{R}$ case we have

$$
\frac{\nu}{2} \int_{-\infty}^{\infty} d q_{1 x} V^{R}\left(\beta, q_{1 x}\right)\left(\frac{\left|q_{1 x}\right|}{Q_{1}^{\mathrm{NP}}}\right)^{\nu C_{1} \lambda^{\mathrm{NP}}} \simeq \frac{1-\nu \lambda^{\mathrm{NP}} C_{1}\left[\ln \left(\bar{\nu} Q_{1}^{\mathrm{NP}}\right)+\chi(\beta)\right]}{1+\beta^{2}}
$$

where $V^{R}$ is given in (A.20). Both in (B.39) and (B.41), corrections are of order $\left(\lambda^{\mathrm{NP}}\right)^{2}$.

\section{The NP functions $\tilde{f}_{a}$}

C.1 $\tilde{f}_{1}$

To compute $\tilde{f}_{1}$ we first split the function $\chi(\beta)$ in the two pieces in (3.2) and evaluate 
the two contributions separately. We have $\tilde{f}_{1}(\nu)=\tilde{f}_{1}^{\prime}(\nu)+\tilde{f}_{1}^{\prime \prime}(\nu)$ where

$$
\begin{aligned}
& \tilde{f}_{1}^{\prime}(\nu)=\int_{-\infty}^{\infty} \prod_{a=2,3} \frac{d \beta_{a} e^{-R_{a}\left(\bar{\nu} \sqrt{1+\beta_{a}^{2}}\right)}}{\pi\left(1+\beta_{a}^{2}\right)} \int_{-\infty}^{\infty} \frac{d \beta \chi^{\prime}(\beta)}{\pi\left(1+\beta^{2}\right)} \mathcal{S}\left(\nu, \beta, \beta_{23}\right), \\
& \tilde{f}_{1}^{\prime \prime}(\nu)=\int_{-\infty}^{\infty} \prod_{a=2,3} \frac{d \beta_{a} e^{-R_{a}\left(\bar{\nu} \sqrt{1+\beta_{a}^{2}}\right)}}{\pi\left(1+\beta_{a}^{2}\right)} \int_{0}^{\infty} \frac{\beta d \beta}{1+\beta^{2}} \mathcal{S}\left(\nu, \beta, \beta_{23}\right) .
\end{aligned}
$$

The evaluation $\tilde{f}_{1}^{\prime}$ is straightforward since all three $\beta$-integrals are rapidly convergent. For large $\nu$ we can factorize the three radiators and the remaining piece is a function of the SL variable $r^{\prime}(\nu, Q)$

$$
\tilde{f}_{1}^{\prime}(\nu)=\prod_{a=1}^{3} e^{-R_{a}(\bar{\nu})} \cdot \mathcal{C}_{1}^{\prime}(\nu), \quad \mathcal{C}_{1}^{\prime}(\nu)=R_{1}^{\prime}(\bar{\nu}) A_{1}\left(\left\{R_{a}^{\prime}(\bar{\nu})\right\}\right) .
$$

For $\nu=0$, we set to zero the radiator and, due to the integration property of $\chi^{\prime}$ in (3.2), we have $\tilde{f}_{1}^{\prime}(0)=0$.

To evaluate $\tilde{f}_{1}^{\prime \prime}$ we observe that the $\beta_{2^{-}}$and $\beta_{3}$-integrals are rapidly convergent, so that the characteristic values of these two variables are $\beta_{2}, \beta_{3}=\mathcal{O}(1)$. On the contrary the $\beta$-integral is logarithmic so that the characteristic values of $\beta$ are large. From (A.16), (A.17) and (A.19), in the region

$$
\beta_{2}, \beta_{3} \ll \beta \simeq \beta_{T}
$$

we have

$$
\mathbf{R}_{1}^{(r)}\left(\bar{\nu}, \beta, \beta_{T}, 0\right) \simeq R_{1}(\bar{\nu} \beta) \quad \mathbf{R}_{1}^{(i)}\left(\bar{\nu}, \beta, \beta_{T}, \gamma\right) \rightarrow 0
$$

(see (A.16) and (A.19)). Therefore, in $\mathcal{S}$ one can factorize the $\# 1$ radiator and the remaining factor is a SL function

$$
\mathcal{S}\left(\nu, \beta, \beta_{23}\right)=e^{-R_{1}(\rho)} \cdot\left\{1+R_{1}^{\prime}(\bar{\nu}) B_{1}\left(\beta, \beta_{23}, R_{1}^{\prime}(\bar{\nu})\right)\right\}, \quad \rho \equiv \bar{\nu} \sqrt{1+\beta^{2}},
$$

with $B_{1}$ vanishing in region (C.3). The first contribution gives the leading contribution to $\tilde{f}_{1}$. We can factorize the $\beta_{2^{-}}$and $\beta_{3}$-integrals and, changing variable from $\beta$ to $\rho$ we obtain

$$
\tilde{f}_{1}(\nu) \simeq \prod_{a=2,3} \int_{-\infty}^{\infty} \frac{d \beta_{a} e^{-R_{a}\left(\bar{\nu} \sqrt{1+\beta_{a}^{2}}\right)}}{\pi\left(1+\beta_{a}^{2}\right)} \int_{\bar{\nu}}^{\infty} \frac{d \rho e^{-R_{1}(\rho)}}{\rho}=\prod_{a=1}^{3} e^{-R_{a}(\bar{\nu})} \mathcal{F}_{2}(\nu) \mathcal{F}_{3}(\nu) E_{1}(\bar{\nu})
$$

where we have factorized the three radiators, the functions $\mathcal{F}_{a}$ are the single jet functions defined in (2.44) and the function $E_{1}$ is introduced in (3.9) and discussed in detail in Appendix D.1. 
Consider the second term contribution in (C.5) to $\tilde{f}_{1}^{\prime \prime}$. It will be denoted by $\tilde{f}_{1}^{\prime \prime \prime}$. Since $B_{1}$ vanishes for large $\beta$, all three $\beta$-integrals are rapidly convergent. As for $\tilde{f}_{1}^{\prime}$, we can factorize the three radiators and the remaining integrals give a SL function

$$
\tilde{f}_{1}^{\prime \prime \prime}(\nu)=\prod_{a=1}^{3} e^{-R_{a}(\bar{\nu})} \cdot \mathcal{C}_{1}^{\prime \prime}(\nu), \quad \mathcal{C}_{1}^{\prime \prime}(\nu)=R_{1}^{\prime}(\bar{\nu}) A_{1}^{\prime}\left(\left\{R_{a}^{\prime}(\bar{\nu})\right\}\right)
$$

Here again we have that $\mathcal{C}_{1}^{\prime \prime}$ vanishes for $R_{1}^{\prime} \rightarrow 0$ and $\nu \rightarrow 0$. Assembling all pieces (C.2), (C.6), and (C.7), the function $\tilde{f}_{1}(\nu)$ is given by

$$
\tilde{f}_{1}(\nu)=\prod_{a=1}^{3} e^{-R_{a}(\bar{\nu})}\left\{\mathcal{F}_{2}(\nu) \mathcal{F}_{3}(\nu) E_{1}(\bar{\nu})+\mathcal{C}_{1}(\nu)\right\}
$$

Here $\mathcal{C}_{1}(\nu)=\mathcal{C}_{1}^{\prime}(\nu)+\mathcal{C}_{1}^{\prime \prime}(\nu)$ is a SL function, which vanishes for $R_{1}^{\prime} \rightarrow 0$ and for $\nu \rightarrow 0$.

To conclude we compute the first loop contribution proportional to $R_{1}^{\prime}$ to $\mathcal{S}$. We have

$$
\mathcal{S}\left(\nu, \beta, \beta_{23}\right) e^{R_{1}(\rho)}=1+\frac{1}{2} R_{1}^{\prime}(\bar{\nu}) \ln \sqrt{\frac{1+\beta^{2}}{1+\beta_{T}^{2}}}+\frac{2}{\pi} \int_{0}^{\infty} \frac{d \gamma}{\gamma} \operatorname{Im}_{1}\left(\bar{\nu}, \beta, \beta_{T}, \gamma\right)+\cdots
$$

The first two terms are the $\gamma=0$ contribution. The last term has been evaluated in [1] and one finds

$$
\frac{2}{\pi} \int_{0}^{\infty} \frac{d \gamma}{\gamma} \operatorname{Im} \mathbf{R}_{1}\left(\bar{\nu}, \beta, \beta_{T}, \gamma\right)=\frac{R_{1}^{\prime}(\bar{\nu})}{2} \cdot \ln \frac{\sqrt{1+\beta^{2}}}{\sqrt{1+\beta_{T}^{2}}} .
$$

Thus the one loop contribution is given by twice the second term. It vanishes for $\beta \rightarrow \infty$.

\section{C.2 $\tilde{f}_{2}$}

As before, to evaluate $\tilde{f}_{2}$ we first split the function $\chi\left(\beta_{2}\right)$ in the two pieces in $(3.2)$ and compute the two contributions. We have $\tilde{f}_{2}(\nu)=\tilde{f}_{2}^{\prime}(\nu)+\tilde{f}_{2}^{\prime \prime}(\nu)$, where

$$
\begin{aligned}
& \tilde{f}_{2}^{\prime}(\nu)=\int_{-\infty}^{\infty} \prod_{a=2,3} \frac{d \beta_{a} e^{-R_{a}\left(\bar{\nu} \sqrt{1+\beta_{a}^{2}}\right)}}{\pi\left(1+\beta_{a}^{2}\right)} \int_{-\infty}^{\infty} \frac{d \beta \mathcal{S}\left(\nu, \beta, \beta_{23}\right)}{\pi\left(1+\beta^{2}\right)} \chi^{\prime}\left(\beta_{2}\right), \\
& \tilde{f}_{2}^{\prime \prime}(\nu)=\int_{0}^{\infty} \frac{\beta_{2} d \beta_{2} e^{-R_{2}\left(\bar{\nu} \sqrt{1+\beta_{2}^{2}}\right)}}{1+\beta_{2}^{2}} \int_{-\infty}^{\infty} \frac{d \beta_{3} e^{-R_{3}\left(\bar{\nu} \sqrt{1+\beta_{3}^{2}}\right)}}{\pi\left(1+\beta_{3}^{2}\right)} \int_{-\infty}^{\infty} \frac{d \beta \mathcal{S}\left(\nu, \beta, \beta_{23}\right)}{\pi\left(1+\beta^{2}\right)} .
\end{aligned}
$$

The analysis of $\tilde{f}_{2}^{\prime}$ is similar to the one of $\tilde{f}_{1}^{\prime}$ performed before and we get

$$
\tilde{f}_{2}^{\prime}(\nu)=\prod_{a=1}^{3} e^{-R_{a}(\bar{\nu})} \cdot \mathcal{C}_{2}^{\prime}(\nu), \quad \mathcal{C}_{2}^{\prime}(\nu)=R_{2}^{\prime}(\bar{\nu}) A_{2}\left(\left\{R_{a}^{\prime}(\bar{\nu})\right\}\right)
$$


where $\mathcal{C}_{2}^{\prime}(\nu)$ is a SL function vanishing for $R_{2}^{\prime} \rightarrow 0$ and $\nu \rightarrow 0$.

To evaluate $\tilde{f}_{2}^{\prime \prime}$ we observe that the $\beta_{3^{-}}$and $\beta$-integrals are rapidly convergent, so that the characteristic values of these two variables are $\beta, \beta_{3}=\mathcal{O}(1)$. On the contrary the $\beta_{2}$-integral is logarithmic so that the characteristic values of $\beta_{2}$ are large. Therefore, to evaluate $\tilde{f}_{2}^{\prime \prime}$ we need to obtain $\mathcal{S}\left(\nu, \beta, \beta_{23}\right)$ in the region

$$
\beta, \beta_{3} \ll \beta_{2}
$$

We show that here one has

$$
\mathcal{S}\left(\nu, \beta, \beta_{23}\right)=e^{-R_{1}\left(\bar{\nu} \sqrt{1+\beta_{2}^{2}}\right)} \cdot\left\{1+R_{1}^{\prime}(\bar{\nu}) B_{2}\left(\nu, \beta, \beta_{23}\right)\right\}
$$

where $B_{2}$ is a SL function of $\nu$ and rapidly vanishes in the region (C.13).

To obtain this result we observe that in this region we must consider also the second term of $\mathcal{S}$ in (3.6) with the $\gamma$-integral and the imaginary part of $\mathbf{R}_{1}$. This integral is logarithmic, as the $\beta_{2}$-integral, so that the characteristic values of $\gamma$ are large compared to $\beta, \beta_{3}$. On the other hand, from (A.16), (A.17) and (A.19), we have $\mathbf{R}_{1}^{(i)}\left(\bar{\nu}, \beta, \beta_{T}, \gamma\right) \rightarrow 0$ for $\beta_{T} \ll \gamma$. We conclude that the region

$$
\beta, \beta_{3} \ll \gamma \ll \beta_{T} \simeq \beta_{2} \simeq \beta_{23}
$$

gives the leading logarithmic approximation of $\tilde{f}_{2}^{\prime \prime}$. We start from this approximation which we shall later improve to account for the first subleading correction $\mathcal{O}\left(R_{1}^{\prime}\right)$. In this region, from (A.16), we have

$$
\begin{aligned}
& \mathbf{R}^{(r)}\left(\bar{\nu}, \beta, \beta_{T}, \gamma\right) \simeq \frac{1}{2} R_{1}(\bar{\nu} \gamma)+\frac{1}{2} R_{1}\left(\bar{\nu} \beta_{2}\right) \\
& \mathbf{R}^{(i)}\left(\bar{\nu}, \beta, \beta_{T}, \gamma\right) \simeq-\frac{\pi}{4} R_{1}^{\prime}(\bar{\nu} \gamma)
\end{aligned}
$$

and, for the two terms of $\mathcal{S}$ in (3.6), we have

$$
\begin{aligned}
e^{-\mathbf{R}_{1}\left(\bar{\nu}, \beta, \beta_{T}, 0\right)} & \simeq e^{-\frac{1}{2} R_{1}\left(\bar{\nu} \beta_{2}\right)} e^{-\frac{1}{2} R_{1}\left(\bar{\nu} \sqrt{1+\beta^{2}}\right)} \\
\int_{0}^{\infty} \frac{d \gamma}{\gamma} \operatorname{Im} e^{-\mathbf{R}_{1}\left(\bar{\nu}, \beta, \beta_{T}, \gamma\right)} & \simeq e^{-\frac{1}{2} R_{1}\left(\bar{\nu} \beta_{2}\right)} \int_{\gamma_{m}}^{\gamma_{M}} \frac{d \gamma}{\gamma} e^{-\frac{1}{2} R_{1}(\bar{\nu} \gamma)} \sin \left(\frac{\pi}{4} R_{1}^{\prime}(\bar{\nu} \gamma)\right)
\end{aligned}
$$

where $\gamma_{M}=\mathcal{O}\left(\beta_{2}\right)$ and $\gamma_{m}=\mathcal{O}(\beta)$. To evaluate the contribution from the imaginary part we first consider

$$
\sin \left(\frac{\pi}{4} R_{1}^{\prime}(\bar{\nu} \gamma)\right)=\frac{\pi}{4} R_{1}^{\prime}(\bar{\nu} \gamma)+\cdots
$$

The terms in the dots can be neglected since they contribute to the integral with terms of order $\alpha_{\mathrm{s}}$. To show this consider the contribution to the $\gamma$-integral from the first term which is of order $\left(\alpha_{\mathrm{s}} \ln \gamma\right)^{3}$ for large $\gamma$. Since the $\gamma$ integration is logarithmic and the radiator is of order $\alpha_{\mathrm{s}} \ln ^{2} \gamma$, the result of the integral is of order $\alpha_{\mathrm{s}}$. 
Taking the first term in (C.18), we find the leading logarithmic contribution

$$
\begin{aligned}
-\frac{2}{\pi} \int_{0}^{\infty} \frac{d \gamma}{\gamma} \operatorname{Im} e^{-\mathbf{R}_{1}\left(\bar{\nu}, \beta, \beta_{T}, \gamma\right)} & \simeq e^{-\frac{1}{2} R_{1}\left(\bar{\nu} \beta_{2}\right)} \int_{\gamma_{m}}^{\gamma_{M}} d \gamma \frac{d}{d \gamma}\left\{e^{-\frac{1}{2} R_{1}(\bar{\nu} \gamma)}\right\} \\
& \simeq e^{-R_{1}\left(\bar{\nu} \beta_{2}\right)}-e^{-\frac{1}{2} R_{1}\left(\bar{\nu} \beta_{2}\right)-\frac{1}{2} R_{1}\left(\bar{\nu} \gamma_{m}\right)}
\end{aligned}
$$

Here we used $\gamma_{M} \sim \beta_{2}$.

In order to keep under control the subleading $\mathcal{O}\left(r^{\prime}\right)$ correction we need to fix precisely the lower integration limit $\gamma_{m}$ in (C.19). To do this it suffices to calculate the exact $\gamma$-integral in the first order in $r^{\prime}$ given in (C.10). Combining (C.19) and (C.10) we have the result

$$
-\frac{2}{\pi} \int_{0}^{\infty} \frac{d \gamma}{\gamma} \operatorname{Im} e^{-\mathbf{R}_{1}\left(\bar{\nu}, \beta, \beta_{T}, \gamma\right)} \simeq e^{-R_{1}\left(\bar{\nu} \sqrt{1+\beta_{2}^{2}}\right)}-e^{-\frac{1}{2} R_{1}\left(\bar{\nu} \sqrt{1+\beta_{2}^{2}}\right)-\frac{1}{2} R_{1}\left(\bar{\nu} \sqrt{1+\beta^{2}}\right)} .
$$

Summing this with the real part contribution in (C.17) we have the cancellation of the leading term $e^{-\frac{1}{2} R_{1}\left(\bar{\nu} \beta_{2}\right)} e^{-\frac{1}{2} R_{1}\left(\bar{\nu} \sqrt{1+\beta^{2}}\right)}$ and we obtain

$$
\mathcal{S}\left(\nu, \beta, \beta_{23}\right) \simeq e^{-R_{1}(\rho)}, \quad \rho \equiv \bar{\nu} \sqrt{1+\beta_{2}^{2}} .
$$

This result shows that if one factorizes in $\mathcal{S}$ the radiator $e^{-R_{1}(\rho)}$, the remaining piece is a SL function and one obtains the final result in (3.15).

The leading piece to $\tilde{f}_{2}$ is obtained from (C.21) and is given by

$$
\tilde{f}_{2}(\nu) \simeq \int \frac{d \beta_{3} e^{-R_{3}\left(\bar{\nu} \sqrt{1+\beta_{3}^{2}}\right)}}{\pi\left(1+\beta_{3}^{2}\right)} \int_{\bar{\nu}}^{\infty} \frac{d \rho}{\rho} e^{-R_{1}(\rho)-R_{2}(\rho)}=\prod_{a=1}^{3} e^{-R_{a}(\bar{\nu})} \cdot \mathcal{F}_{3}(\nu) E_{2}(\bar{\nu})
$$

with $E_{2}$ given in $(3.17)$ and $\mathcal{F}_{3}$ given by $(2.44)$.

The second term in (C.14) (with $B_{2}$ ) gives, after factorizing the radiators, a SL function since all three $\beta$-integrals are rapidly convergent. One gets

$$
\tilde{f}_{2}^{\prime \prime \prime}(\nu)=\prod_{a=1}^{3} e^{-R_{a}(\bar{\nu})} \cdot \mathcal{C}_{2}^{\prime \prime}(\nu), \quad \mathcal{C}_{2}^{\prime \prime}(\nu)=R_{1}^{\prime}(\nu) A_{2}^{\prime}(\nu)
$$

with $A_{2}^{\prime}$ a SL function.

Putting together the various pieces, (C.12), (C.22) and (C.23), we get the final result

$$
\tilde{f}_{2}(\nu)=\prod_{a=1}^{3} e^{-R_{a}(\bar{\nu})} \cdot\left\{\mathcal{F}_{3}(\nu) E_{2}(\nu)+\mathcal{C}_{2}(\nu)\right\}
$$

where $\mathcal{C}_{2}(\nu)=R_{2}^{\prime}(\bar{\nu}) A_{2}(\nu)+R_{1}^{\prime}(\bar{\nu}) A_{2}^{\prime}(\nu)$ is a SL function which vanishes for $r^{\prime} \rightarrow 0$ and $\nu \rightarrow 0$. 


\section{The $E$ functions}

For completeness we recall here the results of an Appendix of Ref. [7] and extend them to the present case.

\section{D.1 $E_{1}$}

We consider first the function $E_{1}(\bar{\nu})$. For the function $E_{1}(\bar{\nu})$ we have the expansion

$$
\begin{aligned}
E_{1}(\bar{\nu}) & \equiv \int_{\bar{\nu}}^{\infty} \frac{d \rho}{\rho} e^{-\left(R_{1}(\rho)-R_{1}(\bar{\nu})\right)} \\
& =\sqrt{\frac{\pi}{2 R_{1}^{\prime \prime}}} N(t)-\frac{R_{1}^{\prime \prime \prime}}{3\left(R_{1}^{\prime \prime}\right)^{2}} X(t)+\mathcal{O}\left(\alpha_{\mathrm{s}}\right), \quad t \equiv \frac{R_{1}^{\prime}}{\sqrt{2 R_{1}^{\prime \prime}}},
\end{aligned}
$$

with $R_{1}^{\prime}, R_{1}^{\prime \prime}, R_{1}^{\prime \prime \prime}$ the first, second and third logarithmic derivatives of $R_{1}$ evaluated at $\bar{\nu}$ and

$$
N(t) \equiv \frac{2}{\sqrt{\pi}} \int_{t}^{\infty} d x e^{-x^{2}+t^{2}}, \quad X(t) \equiv-\frac{\sqrt{\pi}}{8} \frac{d^{3} N(t)}{d t^{3}}=\int_{0}^{\infty} d z z e^{-z-2 t \sqrt{z}},
$$

with the following behaviours

$$
\begin{array}{ll}
t \gg 1: & N(t)=\frac{1}{\sqrt{\pi} t}\left(1-\frac{1}{2 t^{2}}+\frac{3}{t^{4}}+\ldots\right), \quad X(t)=\frac{3}{4 t^{4}}+\mathcal{O}\left(t^{-6}\right) \\
t \ll 1: & N(t)=1-\frac{2 t}{\sqrt{\pi}}+t^{2}-\frac{4 t^{3}}{3 \sqrt{\pi}}+\ldots, \quad X(t)=1-\frac{3 \sqrt{\pi}}{2} t+4 t^{2}+\mathcal{O}\left(t^{3}\right) .
\end{array}
$$

In the region $t=R_{1}^{\prime} / \sqrt{2 R_{1}^{\prime \prime}} \simeq \sqrt{\alpha_{\mathrm{s}}} \ln (\nu Q) \gg 1$, we obtain

$$
E_{1}(\bar{\nu})=\frac{1}{R_{1}^{\prime}(\nu)}\left\{1+\mathcal{O}\left(\frac{1}{\alpha_{\mathrm{s}} \ln ^{2}(\nu Q)}\right)\right\} \text {. }
$$

On the contrary, for $t=R_{1}^{\prime} / \sqrt{2 R_{1}^{\prime \prime}} \simeq \sqrt{\alpha_{\mathrm{s}}} \ln (\nu Q) \ll 1$, we find

$$
E_{1}(\bar{\nu})=\frac{\pi}{2 \sqrt{C_{1} \alpha_{\mathrm{s}}(Q)}}-\ln \left(\bar{\nu} Q_{1}^{\mathrm{PT}}\right)-\frac{\beta_{0}}{6 C_{1}}+\mathcal{O}\left(\sqrt{\alpha_{\mathrm{s}}}\right) .
$$

Notice that the term $\mathcal{O}\left(\sqrt{\alpha_{\mathrm{s}}}\right)$ implicitly includes all corrections $\mathcal{O}\left(\sqrt{\alpha_{\mathrm{s}}}\right), \mathcal{O}\left(\sqrt{\alpha_{\mathrm{s}}} \ln \bar{\nu}\right)$ and $\mathcal{O}\left(\sqrt{\alpha_{\mathrm{s}}} \ln \bar{\nu}^{2}\right)$.

\section{D.2 $E_{a}$ for $a=2,3$}

For the function $E_{a}(\bar{\nu})$ we obtain the following result:

$$
\begin{aligned}
& E_{a}(\bar{\nu}) \equiv \int_{\bar{\nu}}^{\infty} \frac{d \rho}{\rho} e^{-\left(R_{1}(\rho)+R_{a}(\rho)-R_{1}(\bar{\nu})-R_{a}(\bar{\nu})\right)} \\
& =\sqrt{\frac{\pi}{2\left(R_{1}^{\prime \prime}+R_{a}^{\prime \prime}\right)}} N(t)-\frac{R_{1}^{\prime \prime \prime}+R_{a}^{\prime \prime \prime}}{3\left(R_{1}^{\prime \prime}+R_{a}^{\prime \prime}\right)^{2}} X(t)+\mathcal{O}\left(\alpha_{\mathrm{s}}\right), \quad t \equiv \frac{R_{1}^{\prime}+R_{a}^{\prime}}{\sqrt{2\left(R_{1}^{\prime \prime}+R_{a}^{\prime \prime}\right)}}
\end{aligned}
$$


where again $R_{a}^{\prime}, R_{a}^{\prime \prime}, R_{a}^{\prime \prime \prime}$ are the first three derivatives of $R_{a}$ evaluated at $\bar{\nu}$ and the functions $N$ and $X$ are defined in (D.2).

As in the previous case, in the region $\left(R_{1}^{\prime}+R_{a}^{\prime}\right) / \sqrt{2\left(R_{1}^{\prime \prime}+R_{a}^{\prime \prime}\right)} \gg 1$, using the expansions in (D.3), we get

$$
E_{a}(\bar{\nu})=\frac{1}{R_{1}^{\prime}(\nu)+R_{a}^{\prime}(\nu)}\left\{1+\mathcal{O}\left(\frac{1}{\alpha_{\mathrm{s}} \ln ^{2}(\nu Q)}\right)\right\} .
$$

On the contrary, for $\left(R_{1}^{\prime}+R_{a}^{\prime}\right) / \sqrt{2\left(R_{1}^{\prime \prime}+R_{a}^{\prime \prime}\right)} \ll 1$, we obtain, up to contributions $\mathcal{O}\left(\sqrt{\alpha_{\mathrm{s}}}\right)$,

$$
E_{a}(\bar{\nu}) \simeq \frac{\pi}{2 \sqrt{\left(C_{1}+C_{a}\right) \alpha_{\mathrm{s}}(Q)}}-\frac{1}{C_{1}+C_{a}}\left(C_{1} \ln \left(\bar{\nu} Q_{1}^{\mathrm{PT}}\right)+C_{a} \ln \left(\bar{\nu} Q_{a}^{\mathrm{PT}}\right)+\frac{\beta_{0}}{6}\right) .
$$




\section{References}

[1] A. Banfi, Yu.L. Dokshitzer, G. Marchesini and G. Zanderighi, J. High Energy Phys. 07 (2000) 002, [hep-ph/0004027].

[2] S. Catani, L. Trentadue, G. Turnock and B.R. Webber, Nucl. Phys. B 407 (1993) 3;

S. Catani and B.R. Webber, Phys. Lett. B 427 (1998) 377, [hep-ph/9801350];

S. Catani, G. Turnock and B.R. Webber, Phys. Lett. B 295 (1992) 269;

Yu.L. Dokshitzer, A. Lucenti, G. Marchesini and G.P. Salam, J. High Energy Phys. 01 (1998) 011, [hep-ph/9801324].

[3] G.P. Korchemsky and G. Sterman, Nucl. Phys. B 437 (1995) 415 [hep-ph/9411211];

B.R. Webber, Phys. Lett. B 339 (1994) 148 [hep-ph/9408222]; see also Proc. Summer School on Hadronic Aspects of Collider Physics, Zuoz, Switzerland, August 1994, ed. M.P. Locher (PSI, Villigen, 1994) [hep-ph/9411384];

M. Beneke and V.M. Braun, Nucl. Phys. B 454 (1995) 253 [hep-ph/9506452];

Yu.L. Dokshitzer and B.R. Webber, Phys. Lett. B 352 (1995) 451 [hep-ph/9504219];

R. Akhoury and V.I. Zakharov, Phys. Lett. B 357 (1995) 646 [hep-ph/9504248]; Nucl. Phys. B 465 (1996) 295 [hep-ph/9507253];

Yu.L. Dokshitzer, V.A. Khoze and S.I. Troyan, Phys. Rev. D 53 (1996) 89 [hep-ph/9506425];

P. Nason and M.H. Seymour, Nucl. Phys. B 454 (1995) 291 [hep-ph/9506317];

G.P. Korchemsky, G. Oderda and G. Sterman, in Deep Inelastic Scattering and QCD, $5^{\text {th }}$ International Workshop, Chicago, IL, April 1997, p. 988 [hep-ph/9708346];

Yu.L. Dokshitzer, G. Marchesini and B.R. Webber, J. High Energy Phys. 07 (1999) 012 [hep-ph/9905339].

[4] M. Beneke, Phys. Rep. 317 (1999) 1, [hep-ph/9807443].

[5] Yu.L. Dokshitzer, A. Lucenti, G. Marchesini and G.P. Salam, Nucl. Phys. B 511 (1998) 396, [hep-ph/9707532] and Erratum Nucl. Phys. B 593 (2001) 729;

Yu.L. Dokshitzer, A. Lucenti, G. Marchesini and G.P. Salam, J. High Energy Phys. 05 (1998) 003, [hep-ph/9802381].

[6] A. Banfi, Yu.L. Dokshitzer, G. Marchesini and G. Zanderighi, [hep-ph/0010267].

[7] Yu.L. Dokshitzer, G. Marchesini and G.P. Salam, Eur. Phys. J. C 3 (1999) 1, [hep-ph/9812487]. and Erratum Eur. Phys. J. C 1 (2001) 1.

[8] Yu.L. Dokshitzer, G. Marchesini and B.R. Webber, Nucl. Phys. B 469 (1996) 93, [hep-ph/9512336].

[9] G.P.Korchemsky, G.Sterman, Nucl. Phys. B 555 (1999) 335, [hep-ph/9902341];

G.P.Korchemsky, S.Tafat, J. High Energy Phys. 10 (2000) 010, [hep-ph/0007005].

[10] S. Catani, G. Marchesini and B.R. Webber, Nucl. Phys. B 349 (1991) 635.

[11] A. Banfi, G. Salam, G. Zanderighi, under preparation. 
[12] S. Catani and M. Grazzini, Nucl. Phys. B 287 (2000) 570, [hep-ph/9908523], see also A. Banfi, Yu.L. Dokshitzer, G. Marchesini and G. Zanderighi, J. High Energy Phys. 07 (2000) 002, [hep-ph/0004027].

[13] G. Corcella, I.G. Knowles, G. Marchesini, S. Moretti, K. Odagiri, P. Richardson, M.H. Seymour, B.R. Webber, J. High Energy Phys. 01 (2001) 010 [hep-ph/0011363].

[14] S. Catani, Yu.L. Dokshitzer, M. Olsson, G. Turnock and B.R. Webber, Phys. Lett. B 269 (1991) 233. 